\title{
An Interval-Valued Pythagorean Fuzzy Compromise Approach with Correlation-Based Closeness Indices for Multiple- Criteria Decision Analysis of Bridge Construction Methods
}

\author{
Ting-Yu Chen $\mathbb{D}^{1,2,3}$ \\ ${ }^{1}$ Professor, Graduate Institute of Business and Management, College of Management, Chang Gung University, No. 259, \\ Wenhua 1st Rd., Guishan District, Taoyuan City 33302, Taiwan \\ ${ }^{2}$ Adjunct Professor, Department of Industrial and Business Management, College of Management, Chang Gung University, No. 259, \\ Wenhua 1st Rd., Guishan District, Taoyuan City 33302, Taiwan \\ ${ }^{3}$ Adjunct Research Fellow, Department of Nursing, Linkou Chang Gung Memorial Hospital, No. 259, Wenhua 1st Rd., \\ Guishan District, Taoyuan City 33302, Taiwan
}

Correspondence should be addressed to Ting-Yu Chen; tychen@mail.cgu.edu.tw

Received 15 March 2018; Revised 22 August 2018; Accepted 18 September 2018; Published 5 November 2018

Academic Editor: Lucia Valentina Gambuzza

Copyright ( $) 2018$ Ting-Yu Chen. This is an open access article distributed under the Creative Commons Attribution License, which permits unrestricted use, distribution, and reproduction in any medium, provided the original work is properly cited.

\begin{abstract}
The purpose of this paper is to develop a novel compromise approach using correlation-based closeness indices for addressing multiple-criteria decision analysis (MCDA) problems of bridge construction methods under complex uncertainty based on interval-valued Pythagorean fuzzy (IVPF) sets. The assessment of bridge construction methods requires the consideration of multiple alternatives and conflicting tangible and intangible criteria in intricate and varied circumstances. The concept of IVPF sets is capable of handling imprecise and ambiguous information and managing complex uncertainty in real-world applications. Inspired by useful ideas concerning information energies, correlations, and correlation coefficients, this paper constructs new concepts of correlation-based closeness indices for IVPF characteristics and investigates their desirable properties. These indices can be utilized to achieve anchored judgments in decision-making processes and to reflect a certain balance between connections with positive and negative ideal points of reference. Moreover, these indices can fully consider the amount of information associated with higher degrees of uncertainty and effectively fuse imprecise and ambiguous evaluative ratings to construct a meaningful comparison approach. By using the correlation-based closeness index, this paper establishes effective algorithmic procedures of the proposed IVPF compromise approach for conducting multiple-criteria evaluation tasks within IVPF environments. The proposed methodology is implemented in a practical problem of selecting a suitable bridge construction method to demonstrate its feasibility and applicability. The practicality and effectiveness of the proposed methodology are verified through a comparative analysis with well-known compromise methods and other relevant nonstandard fuzzy models.
\end{abstract}

\section{Introduction}

Bridges are a critical part of national development because of their crucial role in road networks. However, compared with other transportation-related constructions, bridges are more prone to environmental impacts. Consequently, bridges are the most fragile component of the transportation system. Damaged or collapsed bridges can result in serious casualties, traffic disruptions, and economic losses. Thus, the development of effective bridge structural designs is extremely important, and the selection of appropriate construction methods is the key to successful bridge construction.

However, the assessment of candidate methods for bridge construction is considered a highly complicated multiplecriteria decision analysis (MCDA) problem. To address this complex MCDA problem, the concept of interval-valued Pythagorean fuzzy (IVPF) sets is applied to describe the fuzziness, ambiguity, and inexactness in the decision-making process according to the degrees of membership and nonmembership that are represented by flexible interval values 
that reflect the degree of hesitation. The aim of this paper is to develop a novel IVPF compromise approach using correlation-based closeness indices to address high degrees of uncertainty when assessing bridge construction methods. Moreover, the proposed methods extend the existing compromise-based methodology to the IVPF context and can be applied to a variety of MCDA fields. In this section, the background, motivation, objective, and contributions of this study are detailed.

1.1. Problem Background of Bridge Construction. Highway and transportation projects can be generally divided into three categories: road engineering, bridge engineering, and tunnel engineering. The construction of bridges is essential for societies to function [1, 2], and their establishment enables transportation among towns, cities, and communities [2]. Almost all developed countries build reliable and durable bridges as a part of their infrastructure [3, 4]. However, bridges are relatively fragile and prone to wear and damage from the environment [5], particularly in regions featuring complex geological structures or natural disasters such as flooding, earthquakes, or typhoons $[6,7]$. Therefore, bridges must be safe and serviceable for users $[8,9]$. As an important part of highway transportation systems, the structural design and construction of bridges are of utmost importance to national development [10, 11]; selecting appropriate construction methods is therefore crucial [6, 12-15].

The structural designing of bridges is divided into two stages. The first stage, the conceptual design stage, primarily involves deciding on the overall structural forms and construction technologies to be adopted and accounting for potential design risks [16]. The second stage is focused on detailed construction analyses [17]. The first stage has a profound effect on the subsequent design process and overall costs [18-20]. In fact, no amount of design detail can make up for poor initial concepts $[19,20]$. Developing effective bridge superstructures in the conceptual design stage has a decisive effect on successful bridge construction [21]. The different construction methods for building bridge superstructures pertain to distinct construction characteristics, applicable environments, construction costs, and construction durations. The types of hazards that can occur because of the construction methods employed and the potential risk factors and preventive measures involved in each construction method also differ. Because bridge projects are large in scale and entail intricate implementation processes, identifying the bridge construction methods that feature the lowest cost, are best matched to local conditions, and are feasible and environmentally friendly has remained the focus of public and private construction industries.

Numerous superstructure construction methods are currently available for bridge projects. However, these methods vary considerably in cost and duration, and the selection of inappropriate methods can lower the quality of the structure, diminish the construction efficiency, and lower the cost-effectiveness of the project [21]. Ensuring the applicability, safety, durability, and cost-effectiveness of bridge structural designs is of utmost importance [22,
23]. The most common accident that occurs during bridge construction is bridge collapse, which is frequently the result of inappropriate construction methods, incurs time and monetary losses, and creates the need to repair environmental damage and undertake subsequent reconstruction [6]. From a durability perspective, bridges are a fragile component in road construction because they are relatively more vulnerable to environmental impact than are the other parts [5]. Accordingly, many assessment criteria must be considered in the bridge design process, such as construction safety, aesthetics, integration with the surrounding environment (both landscaping and ecological maintenance), construction cost efficiency, and operational cost efficiency [9, 13, 16, 22, 24]. These conditions make bridges one of the most challenging and complex structures in construction [9].

In addition to these many assessment criteria, various complex technical and structural problems must be resolved in the design $[9,14,15]$. Decision-makers must consider the safety, maintainability, traffic loads, and structural designs and must demand adequate control over the risk of failure $[16,25]$. To optimize safety assessments, particularly those of highway bridges, accurately estimating the effects of traffic loads on the bridges is crucial [26]. For example, failure to accurately estimate the effect of heavy truckload capacity on the bottom structures of bridges can lead to gradual superstructure collapses and catastrophic accidents [27]. In addition, because severe natural disasters often inflict serious damage, decision-makers must include natural disasters in their bridge design assessments [7]. These uncertainties make the selection problem of bridge construction methods markedly challenging $[12,24]$.

1.2. Motivation and Highlights of the Study. Selecting an appropriate bridge construction method involves numerous and complex criteria and entails challenging technical operations, particularly in regions featuring complicated geological structures or frequent natural disasters $[2,6,14,15,28]$. To illustrate the uncertainties that exist in the intricate decision environment, this study attempts to develop a new MCDA approach involving a novel application of IVPF set theory to describe the uncertainties of decision-making according to the degrees of membership and nonmembership that are represented by flexible interval values that reflect the degree of hesitation. This approach incorporates the compromise model as the basis of construction method development to investigate the related personnel's selection of bridgesuperstructure construction methods in Taiwan. The developed methods and relevant techniques can help decisionmakers navigate the criteria and choose the appropriate bridge construction methods for their particular situations to prevent the many occupational hazards that have occurred during bridge construction over the years. This concern is the first motivation of this paper.

The theory of Pythagorean fuzzy $(\mathrm{PF})$ sets originally introduced by Yager [29-32] is a useful tool to capture the vagueness and uncertainty in decision-making processes [33-36]. PF sets are related to the concept of a membership degree and a nonmembership degree that fulfill a relaxed 
condition that the square sum of the two degrees is less than or equal to one $[34,37-40]$. PF sets have been created as a new and prospective class of nonstandard fuzzy sets because they can accommodate higher degrees of uncertainty compared with other nonstandard fuzzy models [32]. Since Zhang and $\mathrm{Xu}$ [36] initially proposed general mathematical forms of the PS sets, the PF theory has become increasingly popular and widely used in the MCDA field [34, 41]. Furthermore, Zhang [40] generalized PF sets to propose the concept of IVPF sets. IVPF sets permit the degrees of membership and nonmembership of a given set to have an interval value within $[0,1]$; moreover, they are required to satisfy the condition that the square sum of the respective upper bounds of the two intervals is less than or equal to one [40,42, 43]. As an extension of PF sets, IVPF sets have wider application potential because of their superior ability to manage more complex uncertainty and address strong fuzziness, ambiguity, and inexactness in practical situations [42, 44-47].

Many useful decision methods and models have been developed for managing MCDA problems involving IVPF information, such as a linear programming method based on an improved score function for IVPF numbers with partially known weight information [45], a generalized probabilistic IVPF-weighted averaging distance operator [48], new exponential operational laws about IVPF sets and their aggregation operators [46], a new gray relational analysis method based on IVPF Choquet integral average operators [49], new probabilistic aggregation operators with $\mathrm{PF}$ and IVPF information [50], IVPF extended Bonferroni mean operators for dealing with heterogeneous relationships among criteria [47], an IVPF outranking method using a closeness-based assignment model [42], and an extended linear programming technique for the multidimensional analysis of preferences based on IVPF sets [39]. Most existing MCDA methods based on IVPF sets have focused on the investigation of scoring models (e.g., score functions, aggregation operators, and mean operators). Nevertheless, relatively few studies have focused on the development or extensions of the compromise model within the IVPF environment. IVPF sets can provide enough input space for decision-makers to evaluate the assessments with interval numbers [47]; thus, the IVPF theory is a powerful and useful tool for handling fuzziness and vagueness. From this perspective, it would be particularly advantageous to employ the IVPF theory to handle more imprecise and ambiguous information in the selection problem of bridge construction methods, which constitutes the second motivation of this paper.

This paper attempts to incorporate the compromise model as the basis of the developed approach for the extension of the IVPF theory to the compromise-based methodology of application. In numerous real-life decision situations, decision-makers often anchor their subjective judgments with certain points of reference [34, 51-53]. In particular, the specification of these points of reference can influence the intensity or even the rank order of the preferences $[34,54]$, which implies that anchor dependency affects the evaluation outcomes among competing alternatives to some degree. In general, anchor dependency can be effectively achieved with the use of positive and negative ideals [34, 51]. More precisely, human preference can be expressed as an "as close as possible" concept, which utilizes a positive ideal as the point of reference. In contrast, preference can be revealed as an "as far as possible" concept, which employs a negative ideal as the point of reference. As can be expected, the usage of these points of reference affects the contrast of currently achievable performances among competing alternatives $[51,52]$. Under these circumstances, it is essential to incorporate such concepts into the proposed IVPF compromise approach. In other words, it is necessary to address the issue of anchor dependency and locate appropriate positive- and negative-ideal points of reference in the developed approach, which constitutes the third motivation of this paper.

Aiming at addressing the foregoing motivational issues, the purpose of this paper is to propose a simple and effective IVPF compromise approach that works with some interesting concepts (i.e., some comparison measures and indices with respect to points of reference) for addressing MCDA problems of bridge construction methods under complex uncertainty based on IVPF information. In particular, this paper incorporates anchored judgments with displaced and fixed ideals into the modeling process of the developed technique, which is different from the existing MCDA methods in the IVPF context. By using information energies, correlations, and correlation coefficients based on IVPF sets, this paper constructs novel concepts of correlation-based closeness indices to characterize complex IVPF information and reflect a certain balance between the connection with positive-ideal IVPF solutions and the remotest connection with negative-ideal IVPF solutions. Several useful and desirable properties related to these concepts are also explored and discussed to form a solid basis for the proposed methods. This paper develops a new IVPF compromise approach to underlie anchored judgments from opposite viewpoints of displaced and fixed ideals and determine the ultimate priority orders among candidate alternatives for solving MCDA problems involving IVPF information. Two algorithmic procedures are provided to enhance the implementation efficiency of the proposed methods. Moreover, the computations associated with the relevant techniques are simple and effective for facilitating multiple-criteria evaluation tasks in IVPF environments. Based on the flexible and useful IVPF compromise approach, this paper investigates an MCDA problem of bridge construction methods in Taiwan to demonstrate the practical effectiveness of the proposed methods in real-world situations. A comparative analysis with wellknown and widely used compromise models and other MCDA approaches based on relevant nonstandard fuzzy sets is also conducted to validate the reasonability and advantages of the developed methodology.

This paper proposes a novel compromise methodology that fully takes into account a new concept of correlationbased closeness indices instead of the distance measures in classic compromise methods. Until recently, some compromise methods have been employed to investigate relevant issues of bridge design and construction. For example, Mara et al. [28] proposed a joint configuration for panel-level 
connections to compromise the benefit of a rapid fiberreinforced polymer deck installation in bridge construction. Penadés-Plà et al. [2] reviewed different methods and sustainable criteria used for decision-making at each life-cycle phase of a bridge, from design to recycling or demolition. The authors indicated that the decision-making process allows the conversion of a judgment into a rational procedure to reach a compromise solution. Liang et al. [55] applied an extended fuzzy technique for order preference by similarity to ideal solution (TOPSIS) to investigate decision-making schemes in large-scale infrastructure projects. Huang and Wang [56] combined the TOPSIS method and the analytic hierarchy process (AHP) to establish a comparison matrix and applied it to a digital model of road and bridge construction enterprise purchasing. Wang et al. [57] employed the AHP-TOPSIS procedure to develop an optimization decision model for bridge design. Nevertheless, the abovementioned compromise solutions or methods can hardly address the MCDA problem of selecting an appropriate bridge construction method under complex uncertainty. These methods have little capability to model imprecise and uncertain information for an intricate and unpredictable decision environment involving strong fuzziness, ambiguity, and inexactness. To overcome these difficulties, this paper develops a novel compromise model using a useful concept of correlationbased closeness indices to address highly uncertain MCDA problems involving IVPF information and solve the selection problem of bridge construction methods. Particularly, in contrast to the existing compromise-based methodology, the uniqueness of this paper is the consideration of flexible IVPF information in assessing bridge construction methods, the development of new correlation-based closeness indices from the opposite perspectives of displaced and fixed ideals, and the determination of ultimate priority rankings based on a novel IVPF compromise approach.

The remainder of this paper is organized as follows. Section 2 briefly reviews some basic concepts and operations of IVPF sets. Section 3 formulates an MCDA problem within IVPF environments and establishes a novel IVPF compromise approach with correlation-based closeness indices for managing MCDA problems under complex IVPF uncertainty. Section 4 applies the proposed methodology to a real-life MCDA problem of selecting a suitable bridge construction method, along with certain comparative discussions, to demonstrate its feasibility and practicality. To further investigate the application results, Section 5 conducts a comprehensive comparative analysis with well-known compromise methods and with other relevant nonstandard fuzzy models to demonstrate the effectiveness and advantages of the developed approach. Finally, Section 6 presents the conclusions.

\section{Preliminary Definitions}

This section introduces some basic concepts related to PF and IVPF sets that are used throughout this paper. Moreover, selected operations of IVPF values that are helpful in the proposed approach are presented.
Definition 1 (see $[30,32,36])$. A PF set $P$ is defined as a set of ordered pairs of membership and nonmembership in a finite universe of discourse $X$ and is given as follows:

$$
P=\left\{\left\langle x, \mu_{P}(x), v_{P}(x)\right\rangle \mid x \in X\right\}
$$

which is characterized by the degree of membership $\mu_{P}$ : $X \rightarrow[0,1]$ and the degree of nonmembership $v_{P}: X \rightarrow[0,1]$ of the element $x \in X$ in the set $P$ with the condition

$$
0 \leq\left(\mu_{P}(x)\right)^{2}+\left(v_{P}(x)\right)^{2} \leq 1
$$

Let $p=\left(\mu_{P}(x), v_{P}(x)\right)$ denote a PF value. The degree of indeterminacy relative to $P$ for each $x \in X$ is defined as follows:

$$
\pi_{P}(x)=\sqrt{1-\left(\mu_{P}(x)\right)^{2}-\left(\nu_{P}(x)\right)^{2}}
$$

Definition 2 (see $[40,43])$. Let $\operatorname{Int}([0,1])$ denote the set of all closed subintervals of the unit interval $[0,1]$. An IVPF set $\tilde{P}$ is defined as a set of ordered pairs of membership and nonmembership in a finite universe of discourse $X$ and is given as follows:

$$
\tilde{P}=\left\{\left\langle x, \mu_{\tilde{P}}(x), \nu_{\tilde{P}}(x)\right\rangle \mid x \in X\right\}
$$

which is characterized by the interval of the membership degree

$$
\mu_{\tilde{P}}: X \rightarrow \operatorname{Int}([0,1]), x \in X \rightarrow \mu_{\tilde{P}}(x)\left(=\left[\mu_{\tilde{P}}^{-}(x), \mu_{\tilde{P}}^{+}(x)\right]\right) \subseteq[0,1]
$$

and the interval of the nonmembership degree

$v_{\tilde{P}}: X \rightarrow \operatorname{Int}([0,1]), x \in X \rightarrow v_{\tilde{P}}(x)\left(=\left[\nu_{\tilde{P}}^{-}(x), \nu_{\tilde{P}}^{+}(x)\right]\right) \subseteq[0,1]$,

with the following condition:

$$
0 \leq\left(\mu_{\tilde{P}}^{+}(x)\right)^{2}+\left(\nu_{\tilde{P}}^{+}(x)\right)^{2} \leq 1
$$

Let $\quad \tilde{p}=\left(\mu_{\tilde{P}}(x), \nu_{\tilde{P}}(x)\right)=\left(\left[\mu_{\tilde{P}}^{-}(x), \mu_{\tilde{P}}^{+}(x)\right],\left[\nu_{\tilde{P}}^{-}(x), \nu_{\tilde{P}}^{+}(x)\right]\right)$ denote an IVPF value. The interval of the indeterminacy degree relative to $\tilde{P}$ for each $x \in X$ is defined as follows:

$$
\begin{aligned}
\pi_{\tilde{P}}(x) & =\left[\pi_{\tilde{P}}^{-}(x), \pi_{\tilde{P}}^{+}(x)\right] \\
& =\left[\sqrt{1-\left(\mu_{\tilde{P}}^{+}(x)\right)^{2}-\left(v_{\tilde{P}}^{+}(x)\right)^{2}}, \sqrt{1-\left(\mu_{\tilde{P}}^{-}(x)\right)^{2}-\left(\nu_{\tilde{P}}^{-}(x)\right)^{2}}\right] .
\end{aligned}
$$

Definition 3 (see $[40,43])$. Let $\tilde{p}_{1}=\left(\left[\mu_{\tilde{P}_{1}}^{-}(x), \mu_{\tilde{P}_{1}}^{+}(x)\right],\left[v_{\tilde{P}_{1}}^{-}(x)\right.\right.$, $\left.\left.v_{\tilde{P}_{1}}^{+}(x)\right]\right), \tilde{p}_{2}=\left(\left[\mu_{\tilde{P}_{2}}^{-}(x), \mu_{\tilde{P}_{2}}^{+}(x)\right],\left[\nu_{\tilde{P}_{2}}^{-}(x), v_{\tilde{P}_{2}}^{+}(x)\right]\right)$, and $\tilde{p}=\left(\left[\mu_{\tilde{P}}^{-}\right.\right.$ $\left.\left.(x), \mu_{\tilde{P}}^{+}(x)\right],\left[\nu_{\tilde{P}}^{-}(x), v_{\tilde{P}}^{+}(x)\right]\right)$ be three IVPF values in $X$, and let $\alpha \geq 0$. Selected operations are defined as follows: 


$$
\begin{aligned}
& \tilde{p}_{1} V \tilde{p}_{2}=\left(\left[\max \left\{\mu_{\tilde{P}_{1}}^{-}(x), \mu_{\tilde{P}_{2}}^{-}(x)\right\}, \max \left\{\mu_{\tilde{P}_{1}}^{+}(x), \mu_{\tilde{P}_{2}}^{+}(x)\right\}\right],\right. \\
& \left.\left[\min \left\{v_{\tilde{P}_{1}}^{\bar{v}_{1}}(x), v_{\tilde{P}_{2}}^{-}(x)\right\}, \min \left\{v_{\tilde{P}_{1}}^{+}(x), v_{\widetilde{P}_{2}}^{+}(x)\right\}\right]\right), \\
& \tilde{p}_{1} \wedge \tilde{p}_{2}=\left(\left[\min \left\{\mu_{\tilde{P}_{1}}^{-}(x), \mu_{\tilde{P}_{2}}^{-}(x)\right\}, \min \left\{\mu_{\tilde{P}_{1}}^{+}(x), \mu_{\tilde{P}_{2}}^{+}(x)\right\}\right],\right. \\
& \left.\left[\max \left\{v_{\tilde{P}_{1}}^{-}(x), v_{\tilde{P}_{2}}^{-}(x)\right\}, \max \left\{v_{\tilde{P}_{1}}^{+}(x), v_{\widetilde{P}_{2}}^{+}(x)\right\}\right]\right), \\
& \tilde{p}_{1} \oplus \tilde{p}_{2}=\left(\left[\sqrt{\left(\mu_{\tilde{P}_{1}}(x)\right)^{2}+\left(\mu_{\tilde{P}_{2}}(x)\right)^{2}-\left(\mu_{\tilde{P}_{1}}(x)\right)^{2} \cdot\left(\mu_{\tilde{P}_{2}}(x)\right)^{2}},\right.\right. \\
& \left.\sqrt{\left(\mu_{\tilde{P}_{1}}^{+}(x)\right)^{2}+\left(\mu_{\tilde{P}_{2}}^{+}(x)\right)^{2}-\left(\mu_{\tilde{P}_{1}}^{+}(x)\right)^{2} \cdot\left(\mu_{\tilde{P}_{2}}^{+}(x)\right)^{2}}\right], \\
& \left.\left[v_{\tilde{P}_{1}}^{-}(x) \cdot v_{\tilde{P}_{2}}^{-}(x), v_{\tilde{P}_{1}}^{+}(x) \cdot v_{\tilde{P}_{2}}^{+}(x)\right]\right) \text {, } \\
& \tilde{p}_{1} \otimes \tilde{p}_{2}=\left(\left[\mu_{\tilde{P}_{1}}^{-}(x) \cdot \mu_{\tilde{P}_{2}}^{-}(x), \mu_{\tilde{P}_{1}}^{+}(x) \cdot \mu_{\tilde{P}_{2}}^{+}(x)\right],\right. \\
& {\left[\sqrt{\left(v_{\tilde{P}_{1}}^{-}(x)\right)^{2}+\left(v_{\tilde{P}_{2}}^{-}(x)\right)^{2}-\left(v_{\tilde{P}_{1}}^{-}(x)\right)^{2} \cdot\left(v_{\tilde{P}_{2}}^{-}(x)\right)^{2}},\right.} \\
& \left.\left.\sqrt{\left(v_{\vec{P}_{1}}^{+}(x)\right)^{2}+\left(v_{P_{2}}^{+}(x)\right)^{2}-\left(v_{P_{1}}^{+}(x)\right)^{2} \cdot\left(v_{P_{2}}^{+}(x)\right)^{2}}\right]\right), \\
& \tilde{p}^{c}=\left(\left[\nu_{\tilde{P}}^{-}(x), v_{\tilde{P}}^{+}(x)\right],\left[\mu_{\tilde{P}}^{-}(x), \mu_{\tilde{P}}^{+}(x)\right]\right), \\
& \alpha \cdot \tilde{p}=\left(\left[\sqrt{1-\left(1-\left(\mu_{\tilde{P}}^{-}(x)\right)^{2}\right)^{\alpha}}, \sqrt{1-\left(1-\left(\mu_{\tilde{P}}^{+}(x)\right)^{2}\right)^{\alpha}}\right],\right. \\
& \left.\left[\left(v_{\tilde{P}}^{-}(x)\right)^{\alpha},\left(v_{\tilde{P}}^{+}(x)\right)^{\alpha}\right]\right), \\
& (\tilde{p})^{\alpha}=\left(\left[\left(\mu_{\tilde{P}}^{-}(x)\right)^{\alpha},\left(\mu_{\tilde{P}}^{+}(x)\right)^{\alpha}\right],\left[\sqrt{1-\left(1-\left(v_{\tilde{P}}^{-}(x)\right)^{2}\right)^{\alpha}},\right.\right. \\
& \left.\left.\sqrt{1-\left(1-\left(v_{\tilde{P}}^{+}(x)\right)^{2}\right)^{\alpha}}\right]\right) \text {. }
\end{aligned}
$$

Definition 4 (see $[40,43])$. Let $\tilde{p}_{1}$ and $\tilde{p}_{2}$ be two IVPF values in $X$. The distance between $\tilde{p}_{1}$ and $\tilde{p}_{2}$ is defined as follows:

$$
\begin{aligned}
D\left(\tilde{p}_{1}, \tilde{p}_{2}\right)= & \frac{1}{4}\left(\left|\left(\mu_{\tilde{P}_{1}}^{-}(x)\right)^{2}-\left(\mu_{\tilde{P}_{2}}^{-}(x)\right)^{2}\right|+\mid\left(\mu_{\tilde{P}_{1}}^{+}(x)\right)^{2}\right. \\
& -\left(\mu_{\tilde{P}_{2}}^{+}(x)\right)^{2}|+|\left(v_{\tilde{P}_{1}}^{\overline{1}}(x)\right)^{2}-\left(v_{\tilde{P}_{2}}^{-}(x)\right)^{2} \mid \\
& +\left|\left(v_{\tilde{P}_{1}}^{+}(x)\right)^{2}-\left(v_{\tilde{P}_{2}}^{+}(x)\right)^{2}\right|+\mid\left(\pi_{\tilde{P}_{1}}^{-}(x)\right)^{2} \\
& \left.-\left(\pi_{\tilde{P}_{2}}^{-}(x)\right)^{2}|+|\left(\pi_{\tilde{P}_{1}}^{+}(x)\right)^{2}-\left(\pi_{\tilde{P}_{2}}^{+}(x)\right)^{2} \mid\right) .
\end{aligned}
$$

\section{An IVPF Compromise Approach}

This section attempts to propose an effective IVPF compromise approach by means of novel correlation-based closeness indices for addressing MCDA problems within a highly complex uncertain environment based on IVPF sets. This section initially describes an MCDA problem in the IVPF decision context. Based on useful concepts of information energies and correlations for IVPF characteristics, this section establishes novel correlation-based closeness indices from the two different perspectives of displaced and fixed ideals. Some essential and desirable properties are also investigated to furnish a sound basis for the subsequent development of an IVPF compromise approach. Finally, this section provides two algorithmic procedures of the proposed IVPF compromise approach for conducting multiple-criteria evaluation tasks in IVPF environments.

3.1. Problem Formulation. Consider an MCDA problem within the IVPF decision environment. Let $Z=\left\{z_{1}, z_{2}, \cdots\right.$, $\left.z_{m}\right\}$ denote a discrete set of $m(m \geq 2)$ candidate alternatives, and let $C=\left\{c_{1}, c_{2}, \cdots, c_{n}\right\}$ denote a finite set of $n$ $(n \geq 2)$ evaluative criteria. Set $C$ can be generally divided into two sets, $C_{\mathrm{I}}$ and $C_{\mathrm{II}}$, where $C_{\mathrm{I}}$ denotes a collection of benefit criteria (i.e., larger values of $c_{j}$ indicate a higher preference), and $C_{\mathrm{II}}$ denotes a collection of cost criteria (i.e., smaller values of $c_{j}$ indicate a higher preference). Moreover, $C_{\mathrm{I}} \cap C_{\mathrm{II}}=\varnothing$ and $C_{\mathrm{I}} \cup C_{\mathrm{II}}=C$. Let $\mathrm{w}^{T}=\left(w_{1}, w_{2}, \cdots, w_{n}\right)^{T}$ denote the weight vector of $n$ evaluative criteria, where $w_{j} \epsilon$ $[0,1]$ for all $j \in\{1,2, \cdots, n\}$ and $\sum_{j=1}^{n} w_{j}=1$ (i.e., the normalization condition).

The evaluative rating of an alternative $z_{i} \in Z$ in relation to a criterion $c_{j} \in C$ is expressed as an IVPF value $\tilde{p}_{i j}=\left(\left[\mu_{i j}^{-}, \mu_{i j}^{+}\right],\left[v_{i j}^{-}, v_{i j}^{+}\right]\right)$, such that $\left[\mu_{i j}^{-}, \mu_{i j}^{+}\right] \in \operatorname{Int}([0,1]),\left[v_{i j}^{-}\right.$, $\left.v_{i j}^{+}\right] \in \operatorname{Int}([0,1])$, and $0 \leq\left(\mu_{i j}^{+}\right)^{2}+\left(v_{i j}^{+}\right)^{2} \leq 1$. The intervals $\left[\mu_{i j}^{-}, \mu_{i j}^{+}\right]$and $\left[v_{i j}^{-}, v_{i j}^{+}\right]$represent the flexible degrees of membership and nonmembership, respectively, for which $z_{i}$ is evaluated with respect to $c_{j}$. Moreover, the interval of the indeterminacy degree that corresponds to each $\tilde{p}_{i j}$ is determined as $\left[\pi_{i j}^{-}, \pi_{i j}^{+}\right]=$ $\left[\sqrt{1-\left(\mu_{i j}^{+}\right)^{2}-\left(v_{i j}^{+}\right)^{2}}, \sqrt{1-\left(\mu_{i j}^{-}\right)^{2}-\left(v_{i j}^{-}\right)^{2}}\right]$. Accordingly, an MCDA problem involving IVPF information can be concisely expressed in the following IVPF decision matrix:

$$
\tilde{\mathrm{p}}=\left[\tilde{p}_{i j}\right]_{m \times n}=\left[\begin{array}{cccc}
\left(\left[\mu_{11}^{-}, \mu_{11}^{+}\right],\left[v_{11}^{-}, v_{11}^{+}\right]\right) & \left(\left[\mu_{12}^{-}, \mu_{12}^{+}\right],\left[v_{12}^{-}, v_{12}^{+}\right]\right) & \cdots & \left(\left[\mu_{1 n}^{-}, \mu_{1 n}^{+}\right],\left[v_{1 n}^{-}, v_{1 n}^{+}\right]\right) \\
\left(\left[\mu_{21}^{-}, \mu_{21}^{+}\right],\left[v_{21}^{-}, v_{21}^{+}\right]\right) & \left(\left[\mu_{22}^{-}, \mu_{22}^{+}\right],\left[v_{22}^{-}, v_{22}^{+}\right]\right) & \cdots & \left(\left[\mu_{2 n}^{-}, \mu_{2 n}^{+}\right],\left[v_{2 n}^{-}, v_{2 n}^{+}\right]\right) \\
\vdots & \vdots & \ddots & \vdots \\
\left(\left[\mu_{m 1}^{-}, \mu_{m 1}^{+}\right],\left[v_{m 1}^{-}, v_{m 1}^{+}\right]\right) & \left(\left[\mu_{m 2}^{-}, \mu_{m 2}^{+}\right],\left[v_{m 2}^{-}, v_{m 2}^{+}\right]\right) & \cdots & \left(\left[\mu_{m n}^{-}, \mu_{m n}^{+}\right],\left[v_{m n}^{-}, v_{m n}^{+}\right]\right)
\end{array}\right] .
$$


Furthermore, the IVPF characteristics $\tilde{P}_{i}$ of an alternative $z_{i}$ can be represented by all of the relevant IVPF values as follows:

$$
\begin{aligned}
\tilde{P}_{i}= & \left\{\left\langle c_{1}, \tilde{p}_{i 1}\right\rangle,\left\langle c_{2}, \tilde{p}_{i 2}\right\rangle, \cdots,\left\langle c_{n}, \tilde{p}_{\text {in }}\right\rangle\right\} \\
= & \left\{\left\langle c_{1},\left(\left[\mu_{i 1}^{-}, \mu_{i 1}^{+}\right],\left[v_{i 1}^{-}, v_{i 1}^{+}\right]\right)\right\rangle,\left\langle c_{2},\left(\left[\mu_{i 2}^{-}, \mu_{i 2}^{+}\right],\right.\right.\right. \\
& {\left.\left.\left.\left[v_{i 2}^{-}, v_{i 2}^{+}\right]\right)\right\rangle, \cdots,\left\langle c_{n},\left(\left[\mu_{\text {in }}^{-}, \mu_{\text {in }}^{+}\right],\left[\nu_{\text {in }}^{-}, v_{\text {in }}^{+}\right]\right)\right\rangle\right\} . }
\end{aligned}
$$

As explained in the introduction, this paper attempts to locate appropriate positive- and negative-ideal IVPF values as points of reference to concretize anchored judgments in the proposed methodology and handle their influences in decision-making processes. From the two different perspectives of displaced and fixed ideals [34, 51, 54], this paper utilizes the concepts of the displaced/fixed positive- and negative-ideal IVPF solutions. With respect to anchored judgments with displaced ideals, this paper identifies the displaced positive- and negative-ideal IVPF values that are composed of all of the best and worst criterion values attainable, respectively. Usually, the larger the evaluative rating is, the greater the preference is for the benefit criteria and the less the preference is for the cost criteria [42]. Thus, for each benefit criterion, the displaced positiveand negative-ideal IVPF values are designated as the largest and smallest IVPF values, respectively, based on all of the IVPF evaluative ratings $\tilde{p}_{i j}$ in the IVPF decision matrix $\tilde{\mathrm{p}}$. More precisely, $\tilde{p}_{* j}=\vee_{i=1}^{m} \tilde{p}_{i j}$ and $\tilde{p}_{\# j}=\wedge_{i=1}^{m} \tilde{p}_{i j}$ for all $c_{j} \in C_{\mathrm{I}}$ - In contrast, the displaced positive- and negative-ideal IVPF values for each cost criterion are considered the smallest and largest IVPF values, respectively, with respect to all of the criterion-wise evaluative ratings in $\tilde{\mathrm{p}}$. In other words, $\tilde{p}_{* j}=\wedge_{i=1}^{m} \tilde{p}_{i j}$ and $\tilde{p}_{\# j}=\vee_{i=1}^{m} \tilde{p}_{i j}$ for all $c_{j} \in C_{\mathrm{II}}$.

Definition 5. Consider an IVPF decision matrix $\tilde{\mathrm{p}}=\left[\tilde{p}_{i j}\right]_{m \times n}$. Let $z_{*}$ and $z_{\#}$ denote the displaced positive- and negativeideal IVPF solutions, respectively, with respect to $\tilde{\mathrm{p}}$, and their IVPF characteristics $\tilde{P}_{*}$ and $\tilde{P}_{\#}$ are expressed as follows:

$$
\begin{aligned}
& \tilde{P}_{*}=\left\{\left\langle c_{1}, \tilde{p}_{* 1}\right\rangle,\left\langle c_{2}, \tilde{p}_{* 2}\right\rangle, \cdots,\left\langle c_{n}, \tilde{p}_{* n}\right\rangle\right\}, \\
& \tilde{P}_{\#}=\left\{\left\langle c_{1}, \tilde{p}_{\# 1}\right\rangle,\left\langle c_{2}, \tilde{p}_{\# 2}\right\rangle, \cdots,\left\langle c_{n}, \tilde{p}_{\# n}\right\rangle\right\} .
\end{aligned}
$$

Here, $\tilde{p}_{* j}\left(=\left(\left[\mu_{* j}^{-}, \mu_{* j}^{+}\right],\left[v_{* j}^{-}, v_{* j}^{+}\right]\right)\right)$and $\tilde{p}_{\# j}\left(=\left(\left[\mu_{\# j}^{-}, \mu_{\# j}^{+}\right]\right.\right.$, $\left.\left.\left[\nu_{\# j}^{-}, v_{\# j}^{+}\right]\right)\right)$represent the displaced positive- and negativeideal IVPF values, respectively, for each criterion $c_{j} \in C(=$ $C_{\mathrm{I}} \cup C_{\mathrm{II}}$, where $\left.C_{\mathrm{I}} \cap C_{\mathrm{II}}=\varnothing\right)$; they are defined as follows:

$$
\begin{aligned}
& \tilde{p}_{* j}= \begin{cases}\bigvee_{i=1}^{m} \tilde{p}_{i j}=\left(\left[\max _{i=1}^{m} \mu_{i j}^{-}, \max _{i=1}^{m} \mu_{i j}^{+}\right],\left[\min _{i=1}^{m} v_{i j}^{-}, \min _{i=1}^{m} v_{i j}^{+}\right]\right) & \text {if } c_{j} \in C_{\mathrm{I}}, \\
\bigwedge_{i=1}^{m} \tilde{p}_{i j}=\left(\left[\min _{i=1}^{m} \mu_{i j}^{-}, \min _{i=1}^{m} \mu_{i j}^{+}\right],\left[\max _{i=1}^{m} v_{i j}^{-}, \max _{i=1}^{m} v_{i j}^{+}\right]\right) & \text {if } c_{j} \in C_{\mathrm{II}},\end{cases} \\
& \tilde{p}_{\# j}= \begin{cases}\bigwedge_{i=1}^{m} \tilde{p}_{i j}=\left(\left[\min _{i=1}^{m} \mu_{i j}^{-}, \min _{i=1}^{m} \mu_{i j}^{+}\right],\left[\max _{i=1}^{m} v_{i j}^{-}, \max _{i=1}^{m} v_{i j}^{+}\right]\right) & \text {if } c_{j} \in C_{\mathrm{I}}, \\
\bigvee_{i=1}^{m} \tilde{p}_{i j}=\left(\left[\max _{i=1}^{m} \mu_{i j}^{-}, \max _{i=1}^{m} \mu_{i j}^{+}\right],\left[\min _{i=1}^{m} v_{i j}^{-}, \min _{i=1}^{m} v_{i j}^{+}\right]\right) & \text {if } c_{j} \in C_{\mathrm{II}} .\end{cases}
\end{aligned}
$$

Concerning anchored judgments with fixed ideals, the largest IVPF value $([1,1],[0,0])$ and smallest IVPF value $([0,0],[1,1])$ are designated as the fixed positive- and negative-ideal IVPF values, respectively, for each $c_{j} \in C_{\mathrm{I}}$. Conversely, the smallest IVPF value $([0,0],[1,1])$ and largest IVPF value $([1,1],[0,0])$ are considered the fixed positive- and negative-ideal IVPF values, respectively, for each $c_{j} \in C_{\mathrm{II}}$.

Definition 6. Consider an IVPF decision matrix $\tilde{\mathrm{p}}=\left[\tilde{p}_{i j}\right]_{m \times n}$. Let $z_{+}$and $z_{-}$denote the fixed positive- and negative-ideal IVPF solutions, respectively, with respect to $\tilde{\mathrm{p}}$, and their IVPF characteristics $\tilde{P}_{+}$and $\tilde{P}_{-}$are expressed as follows:

$$
\begin{aligned}
& \tilde{P}_{+}=\left\{\left\langle c_{1}, \tilde{p}_{+1}\right\rangle,\left\langle c_{2}, \tilde{p}_{+2}\right\rangle, \cdots,\left\langle c_{n}, \tilde{p}_{+n}\right\rangle\right\}, \\
& \tilde{P}_{-}=\left\{\left\langle c_{1}, \tilde{p}_{-1}\right\rangle,\left\langle c_{2}, \tilde{p}_{-2}\right\rangle, \cdots,\left\langle c_{n}, \tilde{p}_{-n}\right\rangle\right\} .
\end{aligned}
$$

Here, $\tilde{p}_{+j}$ and $\tilde{p}_{-j}$ represent the fixed positive- and negativeideal IVPF values, respectively, for each criterion $c_{j} \in C(=$ $C_{\mathrm{I}} \cup C_{\mathrm{II}}$, where $\left.C_{\mathrm{I}} \cap C_{\mathrm{II}}=\varnothing\right)$; they are defined as follows:

$$
\begin{aligned}
& \tilde{p}_{+j}=\left(\left[\mu_{+j}^{-}, \mu_{+j}^{+}\right],\left[v_{+j}^{-}, v_{+j}^{+}\right]\right)= \begin{cases}([1,1],[0,0]) & \text { if } c_{j} \in C_{\mathrm{I}}, \\
([0,0],[1,1]) & \text { if } c_{j} \in C_{\mathrm{II}},\end{cases} \\
& \tilde{p}_{-j}=\left(\left[\mu_{-j}^{-}, \mu_{-j}^{+}\right],\left[v_{-j}^{-}, v_{-j}^{+}\right]\right)= \begin{cases}([0,0],[1,1]) & \text { if } c_{j} \in C_{\mathrm{I}}, \\
([1,1],[0,0]) & \text { if } c_{j} \in C_{\mathrm{II}} .\end{cases}
\end{aligned}
$$

Note that the respective intervals of the indeterminacy degrees corresponding to $\tilde{p}_{* j}$ and $\tilde{p}_{\# j}$ are given by the following equations: $\left[\pi_{* j}^{-}, \pi_{* j}^{+}\right]=\left[\sqrt{1-\left(\mu_{* j}^{+}\right)^{2}-\left(\nu_{* j}^{+}\right)^{2}}\right.$, 
$\left.\sqrt{1-\left(\mu_{* j}^{-}\right)^{2}-\left(v_{* j}^{-}\right)^{2}}\right]$ and $\left[\pi_{\# j}^{-}, \pi_{\# j}^{+}\right]=\left[\sqrt{1-\left(\mu_{\# j}^{+}\right)^{2}-\left(v_{\# j}^{+}\right)^{2}}\right.$ $\left.\left.\sqrt{1-\left(\mu_{\# j}^{-}\right)^{2}-\left(\nu_{\# j}^{-}\right)^{2}}\right]\right]$. The respective intervals of the indeterminacy degrees corresponding to $\tilde{p}_{+j}$ and $\tilde{p}_{-j}$ are obtained as $\left[\pi_{+j}^{-}, \pi_{+j}^{+}\right]=\left[\pi_{-j}^{-}, \pi_{-j}^{+}\right]=[0,0]$.

3.2. Proposed Methodology. This subsection develops an IVPF compromise approach using a novel concept of correlation-based closeness indices. Considering anchored judgments with the displaced or fixed ideal IVPF solutions, this subsection initially presents useful comparison indices based on information energies and correlations of the IVPF characteristics. Furthermore, two simple and effective algorithmic procedures using the proposed IVPF compromise approach are provided for addressing MCDA problems within the IVPF environment.

In the present study, two useful concepts of correlationbased closeness indices from the different perspectives of displaced and fixed ideals are presented to underlie anchored judgments and to reflect a certain balance between the connection with positive-ideal IVPF solutions and the remotest connection with negative-ideal IVPF solutions. Motivated by the idea of correlation coefficients based on PF sets [37], this paper constructs the novel concept of correlation-based closeness indices in the IVPF context and investigates their useful and desirable properties. These comparison indices provide a solid basis for building subsequent IVPF compromise approaches.

Definition 7. Let $\tilde{p}_{i j}=\left(\left[\mu_{i j}^{-}, \mu_{i j}^{+}\right],\left[v_{i j}^{-}, v_{i j}^{+}\right]\right)$be an IVPF evaluative rating in the IVPF decision matrix $\tilde{\mathrm{p}}$, and let $w_{j}$ be the weight of criterion $c_{j} \in C$. The information energy $E\left(\tilde{P}_{i}\right)$ of the IVPF characteristics $\tilde{P}_{i}$ for each alternative $z_{i} \in Z$ is defined as follows:

$$
\begin{aligned}
E\left(\tilde{P}_{i}\right)= & \frac{1}{2} \sum_{j=1}^{n}\left[w _ { j } \cdot \left(\left(\mu_{i j}^{-}\right)^{4}+\left(\mu_{i j}^{+}\right)^{4}+\left(v_{i j}^{-}\right)^{4}+\left(v_{i j}^{+}\right)^{4}\right.\right. \\
& \left.\left.+\left(\pi_{i j}^{-}\right)^{4}+\left(\pi_{i j}^{+}\right)^{4}\right)\right] .
\end{aligned}
$$

Theorem 1. The information energy $E\left(\tilde{P}_{i}\right)$ of the IVPF characteristics $\tilde{P}_{i}$ in $\tilde{\mathrm{p}}$ satisfies the following properties:

$$
\begin{aligned}
& \text { (T1.1) } 0<E\left(\tilde{P}_{i}\right) \leq 1 \\
& \left(\text { T1.2) } E\left(\tilde{P}_{+}\right)=E\left(\tilde{P}_{-}\right)=1\right.
\end{aligned}
$$

Proof. (T1.1) Because $\tilde{p}_{i j}$ is an IVPF value, the following are known: $\left(\mu_{i j}^{+}\right)^{2}+\left(v_{i j}^{+}\right)^{2}+\left(\pi_{i j}^{-}\right)^{2}=1$ and $\left(\mu_{i j}^{-}\right)^{2}+\left(v_{i j}^{-}\right)^{2}+$ $\left(\pi_{i j}^{+}\right)^{2}=1$. Thus, $0.5 \cdot\left(\left(\mu_{i j}^{-}\right)^{2}+\left(\mu_{i j}^{+}\right)^{2}+\left(v_{i j}^{-}\right)^{2}+\left(v_{i j}^{+}\right)^{2}+\left(\pi_{i j}^{-}\right)^{2}+\right.$ $\left.\left(\pi_{i j}^{+}\right)^{2}\right)=1$. With these results, it is readily proved that $0<0.5 \cdot\left(\left(\mu_{i j}^{-}\right)^{4}+\left(\mu_{i j}^{+}\right)^{4}+\left(v_{i j}^{-}\right)^{4}+\left(v_{i j}^{+}\right)^{4}+\left(\pi_{i j}^{-}\right)^{4}+\left(\pi_{i j}^{+}\right)^{4}\right) \leq 1$.
Using the normalization condition of criterion weights $\sum_{j=1}^{n}$ $w_{j}=1$, one can then obtain $0<E\left(\tilde{P}_{i}\right) \leq 1$; i.e., (T1.1) is valid.

(T1.2) According to Definition 6, one has $\tilde{P}_{+}=\left\{\left(\left\langle c_{j},([1\right.\right.\right.$, $\left.\left.1],[0,0])\rangle \mid c_{j} \in C_{\mathrm{I}}\right),\left(\left\langle c_{j},([0,0],[1,1])\right\rangle \mid c_{j} \in C_{\mathrm{II}}\right)\right\}$ and $\tilde{P}_{-}=$ $\left\{\left(\left\langle c_{j},([0,0],[1,1])\right\rangle \mid c_{j} \in C_{\mathrm{I}}\right),\left(\left\langle c_{j},([1,1],[0,0])\right\rangle \mid c_{j} \in C_{\mathrm{II}}\right)\right\}$. Therefore, one can easily infer that $E\left(\tilde{P}_{+}\right)=E\left(\tilde{P}_{-}\right)=\sum_{j=1}^{n}$ $w_{j}=1$. This establishes the theorem.

Definition 8. Let $\tilde{P}_{i}$ and $\tilde{P}_{i^{\prime}}$ be two IVPF characteristics in $\tilde{\mathrm{p}}$, and let $w_{j}$ be the weight of $c_{j} \in C$. The correlation $R$ between $\tilde{P}_{i}$ and $\tilde{P}_{i^{\prime}}$ is defined as follows:

$$
\begin{aligned}
R\left(\tilde{P}_{i}, \tilde{P}_{i^{\prime}}\right)= & \frac{1}{2} \sum_{j=1}^{n}\left[w _ { j } \cdot \left(\left(\mu_{i j}^{-}\right)^{2} \cdot\left(\mu_{i^{\prime} j}^{-}\right)^{2}+\left(\mu_{i j}^{+}\right)^{2} \cdot\left(\mu_{i^{\prime} j}^{+}\right)^{2}\right.\right. \\
& +\left(v_{i j}^{-}\right)^{2} \cdot\left(v_{i^{\prime} j}^{-}\right)^{2}+\left(v_{i j}^{+}\right)^{2} \cdot\left(v_{i^{\prime} j}^{+}\right)^{2}+\left(\pi_{i j}^{-}\right)^{2} \\
& \left.\left.\cdot\left(\pi_{i^{\prime} j}^{-}\right)^{2}+\left(\pi_{i j}^{+}\right)^{2} \cdot\left(\pi_{i^{\prime} j}^{+}\right)^{2}\right)\right] .
\end{aligned}
$$

Theorem 2. The correlation $R\left(\tilde{P}_{i}, \tilde{P}_{i^{\prime}}\right)$ between two IVPF characteristics $\tilde{P}_{i}$ and $\tilde{P}_{i^{\prime}}$ in $\tilde{\mathrm{p}}$ satisfies the following properties:

$$
\begin{aligned}
& \text { (T2.1) } R\left(\tilde{P}_{i}, \tilde{P}_{i}\right)=E\left(\tilde{P}_{i}\right) \\
& \text { (T2.2) } 0 \leq R\left(\tilde{P}_{i}, \tilde{P}_{i^{\prime}}\right) \leq 1 \\
& \text { (T2.3) } R\left(\tilde{P}_{i}, \tilde{P}_{i^{\prime}}\right)=R\left(\tilde{P}_{i^{\prime}}, \tilde{P}_{i}\right) \\
& \text { (T2.4) } R\left(\tilde{P}_{i}, \tilde{P}_{+}\right)=1 / 2\left\{\sum_{c_{j} \in C_{\mathrm{I}}}\left[w_{j} \cdot\left(\left(\mu_{i j}^{-}\right)^{2}+\left(\mu_{i j}^{+}\right)^{2}\right)\right]+\right. \\
&\left.\sum_{c_{j} \in C_{\text {II }}}\left[w_{j} \cdot\left(\left(v_{i j}^{-}\right)^{2}+\left(v_{i j}^{+}\right)^{2}\right)\right]\right\} \\
& \text { (T2.5) } R\left(\tilde{P}_{i}, \tilde{P}_{-}\right)=1 / 2\left\{\sum_{c_{j} \in C_{\mathrm{I}}}\left[w_{j} \cdot\left(\left(v_{i j}^{-}\right)^{2}+\left(v_{i j}^{+}\right)^{2}\right)\right]+\right. \\
&\left.\sum_{c_{j} \in C_{\text {II }}}\left[w_{j} \cdot\left(\left(\mu_{i j}^{-}\right)^{2}+\left(\mu_{i j}^{+}\right)^{2}\right)\right]\right\} .
\end{aligned}
$$

Proof. (T2.1)-(T2.3) are evident. (T2.4) and (T2.5) are straightforward because $\tilde{p}_{+j}=([1,1],[0,0])$ and $\tilde{p}_{-j}=([0,0]$, $[1,1])$ for $c_{j} \in C_{\mathrm{I}}, \tilde{p}_{+j}=([0,0],[1,1])$ and $\tilde{p}_{-j}=([1,1],[0,0])$ for $c_{j} \in C_{\mathrm{II}}$, and $\left[\pi_{+j}^{-}, \pi_{+j}^{+}\right]=\left[\pi_{-j}^{-}, \pi_{-j}^{+}\right]=[0,0]$ for $c_{j} \in C$. This completes the proof.

Definition 9. Let $\tilde{P}_{i}$ and $\tilde{P}_{i^{\prime}}$ be two IVPF characteristics in $\tilde{\mathrm{p}}$. The correlation coefficient $K$ between $\tilde{P}_{i}$ and $\tilde{P}_{i^{\prime}}$ is defined as follows:

$$
K\left(\tilde{P}_{i}, \tilde{P}_{i^{\prime}}\right)=\frac{R\left(\tilde{P}_{i}, \tilde{P}_{i^{\prime}}\right)}{\sqrt{E\left(\tilde{P}_{i}\right) \cdot E\left(\tilde{P}_{i^{\prime}}\right)}} .
$$

Theorem 3. The correlation coefficient $K\left(\tilde{P}_{i}, \tilde{P}_{i^{\prime}}\right)$ between two IVPF characteristics $\tilde{P}_{i}$ and $\tilde{P}_{i^{\prime}}$ satisfies the following properties: 
(T3.1) $K\left(\tilde{P}_{i}, \tilde{P}_{i^{\prime}}\right)=K\left(\tilde{P}_{i^{\prime}}, \tilde{P}_{i}\right)$

(T3.2) $0 \leq K\left(\tilde{P}_{i}, \tilde{P}_{i^{\prime}}\right) \leq 1$

(T3.3) $K\left(\tilde{P}_{i}, \tilde{P}_{i^{\prime}}\right)=1$ if $\tilde{P}_{i}=\tilde{P}_{i^{\prime}}$

Proof. (T3.1) is trivial.

(T3.2) It can be easily obtained that $K\left(\tilde{P}_{i}, \tilde{P}_{i^{\prime}}\right) \geq 0$ based on the properties in (T1.1) (i.e., $\left.0<E\left(\tilde{P}_{i}\right), E\left(\tilde{P}_{i^{\prime}}\right) \leq 1\right)$ and (T2.2) (i.e., $\left.0 \leq R\left(\tilde{P}_{i}, \tilde{P}_{i^{\prime}}\right) \leq 1\right)$. By using the Cauchy-Schwarz inequality, the following relationship can be determined:

$$
\begin{aligned}
& {\left[\left(\sqrt[4]{w_{j}} \cdot \mu_{i j}^{-}\right)^{2} \cdot\left(\sqrt[4]{w_{j}} \cdot \mu_{i^{\prime} j}^{-}\right)^{2}+\left(\sqrt[4]{w_{j}} \cdot \mu_{i j}^{+}\right)^{2} \cdot\left(\sqrt[4]{w_{j}} \cdot \mu_{i^{\prime} j}^{+}\right)^{2}\right.} \\
& +\left(\sqrt[4]{w_{j}} \cdot v_{i j}^{-}\right)^{2} \cdot\left(\sqrt[4]{w_{j}} \cdot v_{i^{\prime} j}^{-}\right)^{2}+\left(\sqrt[4]{w_{j}} \cdot v_{i j}^{+}\right)^{2} \cdot\left(\sqrt[4]{w_{j}} \cdot v_{i^{\prime} j}^{+}\right)^{2} \\
& \left.+\left(\sqrt[4]{w_{j}} \cdot \pi_{i j}^{-}\right)^{2} \cdot\left(\sqrt[4]{w_{j}} \cdot \pi_{i^{\prime} j}^{-}\right)^{2}+\left(\sqrt[4]{w_{j}} \cdot \pi_{i j}^{+}\right)^{2} \cdot\left(\sqrt[4]{w_{j}} \cdot \pi_{i^{\prime} j}^{+}\right)^{2}\right]^{2} \\
& \leq\left\{\left[\left(\sqrt[4]{w_{j}} \cdot \mu_{i j}^{-}\right)^{2}\right]^{2}+\left[\left(\sqrt[4]{w_{j}} \cdot \mu_{i j}^{+}\right)^{2}\right]^{2}+\left[\left(\sqrt[4]{w_{j}} \cdot v_{i j}^{-}\right)^{2}\right]^{2}\right. \\
& \left.+\left[\left(\sqrt[4]{w_{j}} \cdot v_{i j}^{+}\right)^{2}\right]^{2}+\left[\left(\sqrt[4]{w_{j}} \cdot \pi_{i j}^{-}\right)^{2}\right]^{2}+\left[\left(\sqrt[4]{w_{j}} \cdot \pi_{i j}^{+}\right)^{2}\right]^{2}\right\} \\
& +\left\{\left[\left(\sqrt[4]{w_{j}} \cdot \mu_{i^{\prime} j}^{-}\right)^{2}\right]^{2}+\left[\left(\sqrt[4]{w_{j}} \cdot \mu_{i^{\prime} j}^{+}\right)^{2}\right]^{2}+\left[\left(\sqrt[4]{w_{j}} \cdot v_{i^{\prime} j}^{-}\right)^{2}\right]^{2}\right. \\
& \left.+\left[\left(\sqrt[4]{w_{j}} \cdot v_{i^{\prime} j}^{+}\right)^{2}\right]^{2}+\left[\left(\sqrt[4]{w_{j}} \cdot \pi_{i^{\prime} j}^{-}\right)^{2}\right]^{2}+\left[\left(\sqrt[4]{w_{j}} \cdot \pi_{i^{\prime} j}^{+}\right)^{2}\right]^{2}\right\} \\
& =\left(w_{j} \cdot\left(\mu_{i j}^{-}\right)^{4}+w_{j} \cdot\left(\mu_{i j}^{+}\right)^{4}+w_{j} \cdot\left(v_{i j}^{-}\right)^{4}+w_{j} \cdot\left(v_{i j}^{+}\right)^{4}+w_{j}\right. \\
& \left.+\left(\pi_{i j}^{-}\right)^{4}+w_{j} \cdot\left(\pi_{i j}^{+}\right)^{4}\right) \cdot\left(w_{j} \cdot\left(\mu_{i^{\prime} j}^{-}\right)^{4}+w_{j} \cdot\left(\mu_{i^{\prime} j}^{+}\right)^{4}+w_{j}\right. \\
& \left.+\left(v_{i^{\prime} j}^{-}\right)^{4}+w_{j} \cdot\left(v_{i^{\prime} j}^{+}\right)^{4}+w_{j} \cdot\left(\pi_{i^{\prime} j}^{-}\right)^{4}+w_{j} \cdot\left(\pi_{i^{\prime} j}^{+}\right)^{4}\right) \\
& =\left[w_{j} \cdot\left(\left(\mu_{i j}^{-}\right)^{4}+\left(\mu_{i j}^{+}\right)^{4}+\left(v_{i j}^{-}\right)^{4}+\left(v_{i j}^{+}\right)^{4}+\left(\pi_{i j}^{-}\right)^{4}+\left(\pi_{i j}^{+}\right)^{4}\right)\right] \\
& +\left[w _ { j } \cdot \left(\left(\mu_{i^{\prime} j}^{-}\right)^{4}+\left(\mu_{i^{\prime} j}^{+}\right)^{4}+\left(v_{i^{\prime} j}^{-}\right)^{4}+\left(v_{i^{\prime} j}^{+}\right)^{4}+\left(\pi_{i^{\prime} j}^{-}\right)^{4}\right.\right. \\
& \left.\left.+\left(\pi_{i^{\prime} j}^{+}\right)^{4}\right)\right] \cdot
\end{aligned}
$$

With the above results, one can employ Definition 8 to infer the following:

$$
\begin{aligned}
\left(R\left(\tilde{P}_{i}, \tilde{P}_{i^{\prime}}\right)\right)^{2}= & \left\{\frac { 1 } { 2 } \sum _ { j = 1 } ^ { n } \left[w _ { j } \cdot \left(\left(\mu_{i j}^{-}\right)^{2} \cdot\left(\mu_{i^{\prime} j}^{-}\right)^{2}+\left(\mu_{i j}^{+}\right)^{2} \cdot\left(\mu_{i^{\prime} j}^{+}\right)^{2}\right.\right.\right. \\
& +\left(v_{i j}^{-}\right)^{2} \cdot\left(v_{i^{\prime} j}^{-}\right)^{2}+\left(v_{i j}^{+}\right)^{2} \cdot\left(v_{i^{\prime} j}^{+}\right)^{2}+\left(\pi_{i j}^{-}\right)^{2} \\
& \left.\left.\left.\cdot\left(\pi_{i^{\prime} j}^{-}\right)^{2}+\left(\pi_{i j}^{+}\right)^{2} \cdot\left(\pi_{i^{\prime} j}^{+}\right)^{2}\right)\right]\right\}
\end{aligned}
$$

$$
\begin{aligned}
= & \left(\frac{1}{2}\right)^{2} \cdot\left\{\sum _ { j = 1 } ^ { n } \left[\left(\sqrt[4]{w_{j}} \cdot \mu_{i j}^{-}\right)^{2} \cdot\left(\sqrt[4]{w_{j}} \cdot \mu_{i^{\prime} j}^{-}\right)^{2}\right.\right. \\
& +\left(\sqrt[4]{w_{j}} \cdot \mu_{i j}^{+}\right)^{2} \cdot\left(\sqrt[4]{w_{j}} \cdot \mu_{i^{\prime} j}^{+}\right)^{2}+\left(\sqrt[4]{w_{j}} \cdot v_{i j}^{-}\right)^{2} \\
& \cdot\left(\sqrt[4]{w_{j}} \cdot v_{i^{\prime} j}^{-}\right)^{2}+\left(\sqrt[4]{w_{j}} \cdot v_{i j}^{+}\right)^{2} \cdot\left(\sqrt[4]{w_{j}} \cdot v_{i^{\prime} j}^{+}\right)^{2} \\
& +\left(\sqrt[4]{w_{j}} \cdot \pi_{i j}^{-}\right)^{2} \cdot\left(\sqrt[4]{w_{j}} \cdot \pi_{i^{\prime} j}^{-}\right)^{2}+\left(\sqrt[4]{w_{j}} \cdot \pi_{i j}^{+}\right)^{2} \\
& \left.\left.+\left(\sqrt[4]{w_{j}} \cdot \pi_{i^{\prime} j}^{+}\right)^{2}\right]\right\}^{2} \leq\left(\frac{1}{2}\right)^{2} \sum_{j=1}^{n}\left[w _ { j } \cdot \left(\left(\mu_{i j}^{-}\right)^{4}\right.\right. \\
& \left.\left.+\left(\mu_{i j}^{+}\right)^{4}+\left(v_{i j}^{-}\right)^{4}+\left(v_{i j}^{+}\right)^{4}+\left(\pi_{i j}^{-}\right)^{4}+\left(\pi_{i j}^{+}\right)^{4}\right)\right] \\
& \cdot \sum_{j=1}^{n}\left[w _ { j } \cdot \left(\left(\mu_{i^{\prime} j}^{-}\right)^{4}+\left(\mu_{i^{\prime} j}^{+}\right)^{4}+\left(v_{i^{\prime} j}^{-}\right)^{4}+\left(v_{i^{\prime} j}^{+}\right)^{4}\right.\right. \\
& \left.\left.+\left(\pi_{i^{\prime} j}^{-}\right)^{4}+\left(\pi_{i^{\prime} j}^{+}\right)^{4}\right)\right]=\left\{\frac { 1 } { 2 } \sum _ { j = 1 } ^ { n } \left[w _ { j } \cdot \left(\left(\mu_{i j}^{-}\right)^{4}\right.\right.\right. \\
& \left.\left.\left.+\left(\mu_{i^{\prime} j}^{+}\right)^{4}+\left(v_{i^{\prime}}^{-}\right)^{4}+\left(v_{i j}^{+}\right)^{4}+\left(\pi_{i j}^{-}\right)^{4}+\left(\pi_{i j}^{+}\right)^{4}\right)\right]\right\} \\
& +\left\{\frac { 1 } { 2 } \sum _ { j = 1 } ^ { n } \left[w _ { j } \cdot \left(\left(\mu_{i^{\prime} j}^{-}\right)^{4}+\left(\mu_{i^{\prime} j}^{+}\right)^{4}+\left(v_{i^{\prime} j}^{-}\right)^{4}\right.\right.\right. \\
& \left.\left.\left.+\left(v_{i^{\prime} j}^{+}\right)^{4}+\left(\pi_{i^{\prime} j}^{-}\right)^{4}+\left(\pi_{i^{\prime} j}^{+}\right)^{4}\right)\right]\right\}=E\left(\tilde{P}_{i}\right) \cdot E\left(\tilde{P}_{i^{\prime}}\right) .
\end{aligned}
$$

The obtained inequality can be written as $R\left(\tilde{P}_{i}, \tilde{P}_{i^{\prime}}\right) \leq$ $\sqrt{E\left(\tilde{P}_{i}\right) \cdot E\left(\tilde{P}_{i^{\prime}}\right)}$ because $R\left(\tilde{P}_{i}, \tilde{P}_{i^{\prime}}\right) \geq 0, E\left(\tilde{P}_{i}\right)>0$, and $E\left(\tilde{P}_{i^{\prime}}\right)>$ 0 . Thus, we conclude that $K\left(\tilde{P}_{i}, \tilde{P}_{i^{\prime}}\right) \leq 1$; i.e., (T3.2) is valid.

(T3.3) It is known that $E\left(\tilde{P}_{i}\right)=E\left(\tilde{P}_{i^{\prime}}\right)$ because $\tilde{P}_{i}=\tilde{P}_{i^{\prime}}$. Using (T2.1) (i.e., $\left.R\left(\tilde{P}_{i}, \tilde{P}_{i}\right)=E\left(\tilde{P}_{i}\right)\right)$ yields $K\left(\tilde{P}_{i}, \tilde{P}_{i^{\prime}}\right)=E\left(\tilde{P}_{i}\right) /$ $\sqrt{E\left(\tilde{P}_{i}\right) \cdot E\left(\tilde{P}_{i}\right)}=1$. This basis establishes the theorem.

Turning now to the issue of identifying appropriate comparison indices in the proposed IVPF compromise approach, this paper develops a useful concept of correlation-based closeness indices to provide a starting point for realizing anchored judgments with displaced/fixed ideals in the MCDA process. First, this paper incorporates the displaced positive-ideal IVPF solution $z_{*}$ and the displaced negativeideal IVPF solution $z_{\#}$ into the specification of correlationbased closeness indices. As mentioned earlier, the selection of anchor values would influence the intensity of the preferences about candidate alternatives. In this respect, the larger $K\left(\tilde{P}_{i}, \tilde{P}_{*}\right)$ is, the better are the IVPF characteristics $\tilde{P}_{i}$. Furthermore, the smaller $K\left(\tilde{P}_{i}, \tilde{P}_{\#}\right)$ is, the better are the $\tilde{P}_{i}$. The IVPF characteristic most associated with $\tilde{P}_{*}$ does not

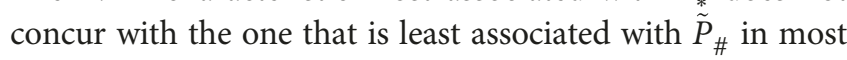
real-world decision situations. To address this issue, this 
paper defines a novel correlation-based closeness index $I_{d}$ to simultaneously measure the strength of the association with $\tilde{P}_{*}$ and $\tilde{P}_{\#}$. The usage of the index $I_{d}$ can facilitate anchored judgments with displaced ideals. Moreover, $I_{d}$ is an effective comparison index that can reflect some balance between the connection with $z_{*}$ and the remotest connection with $z_{\#}$.

Definition 10. Let $\tilde{P}_{i}$ be the IVPF characteristics of alternative $z_{i} \in Z$ in $\tilde{\mathrm{p}}$. With respect to the displaced ideal IVPF solutions $z_{*}$ and $z_{\#}$, the correlation-based closeness index $I_{d}$ of $\tilde{P}_{i}$ is defined as follows:

$$
I_{d}\left(\tilde{P}_{i}\right)=\frac{K\left(\tilde{P}_{i}, \tilde{P}_{*}\right)}{K\left(\tilde{P}_{i}, \tilde{P}_{*}\right)+K\left(\tilde{P}_{i}, \tilde{P}_{\#}\right)} .
$$

Theorem 4. For each IVPF characteristic $\tilde{P}_{i}$ in $\tilde{\mathrm{p}}$, the correlation-based closeness index $I_{d}$ satisfies the property $0 \leq$ $I_{d}\left(\tilde{P}_{i}\right) \leq 1$.

Proof. Using (T3.2), the proof of this property is direct because $K\left(\tilde{P}_{i}, \tilde{P}_{*}\right) \geq 0, K\left(\tilde{P}_{i}, \tilde{P}_{\#}\right) \geq 0$, and $K\left(\tilde{P}_{i}, \tilde{P}_{*}\right) \leq K\left(\tilde{P}_{i}\right.$, $\left.\tilde{P}_{*}\right)+K\left(\tilde{P}_{i}, \tilde{P}_{\#}\right)$.

Next, consider anchored judgments with fixed ideals. As described in Definition 6, the fixed positive-ideal IVPF solution $z_{+}$is composed of the largest IVPF value $([1,1]$, $[0,0])$ for each benefit criterion $c_{j} \in C_{\mathrm{I}}$ and the smallest $\operatorname{IVPF}$ value $([0,0],[1,1])$ for each cost criterion $c_{j} \in C_{\mathrm{II}}$.
In contrast, the fixed negative-ideal IVPF solution $z_{-}$is composed of the smallest IVPF value $([0,0],[1,1])$ for each $c_{j} \in C_{\mathrm{I}}$ and the largest IVPF value $([1,1],[0,0])$ for each $c_{j} \in C_{\mathrm{II}}$. For the most part, the larger $K\left(\tilde{P}_{i}, \tilde{P}_{+}\right)$is, the better are the IVPF characteristics $\tilde{P}_{i}$; moreover, the smaller $K\left(\tilde{P}_{i}, \tilde{P}_{-}\right)$is, the better are the $\tilde{P}_{i}$. However, a specific $\tilde{P}_{i}$ that is most associated with $\tilde{P}_{+}$does not concur with the one that is least associated with $\tilde{P}_{-}$. For this reason, this paper provides another comparison index to effectively underlie anchored judgments with the fixed positive- and negative-ideal IVPF solutions. As indicated in the following definition, this paper develops a useful correlation-based closeness index $I_{f}$ of $\tilde{P}_{i}$ to simultaneously measure the strength of the association with $\tilde{P}_{+}$and $\tilde{P}_{-}$to achieve a certain balance between the connection with $z_{+}$and the remotest connection with $z_{-}$.

Definition 11. Let $\tilde{P}_{i}$ be the IVPF characteristics of alternative $z_{i} \in Z$ in $\tilde{\mathrm{p}}$. With respect to the fixed ideal IVPF solutions $z_{+}$ and $z_{-}$, the correlation-based closeness index $I_{f}$ of $\tilde{P}_{i}$ is defined as follows:

$$
I_{f}\left(\tilde{P}_{i}\right)=\frac{K\left(\tilde{P}_{i}, \tilde{P}_{+}\right)}{K\left(\tilde{P}_{i}, \tilde{P}_{+}\right)+K\left(\tilde{P}_{i}, \tilde{P}_{-}\right)} .
$$

Theorem 5. For each IVPF characteristic $\tilde{P}_{i}$ in $\tilde{\mathrm{p}}$, the correlation-based closeness index $I_{f}\left(\tilde{P}_{i}\right)$ based on the fixed ideal IVPF solutions can be determined as follows:

$$
I_{f}\left(\tilde{P}_{i}\right)=\frac{\sum_{c_{j} \in C_{\mathrm{I}}}\left[w_{j} \cdot\left(\left(\mu_{i j}^{-}\right)^{2}+\left(\mu_{i j}^{+}\right)^{2}\right)\right]+\sum_{c_{j} \in C_{\mathrm{II}}}\left[w_{j} \cdot\left(\left(v_{i j}^{-}\right)^{2}+\left(v_{i j}^{+}\right)^{2}\right)\right]}{\sum_{j=1}^{n}\left[w_{j} \cdot\left(2-\left(\pi_{i j}^{-}\right)^{2}-\left(\pi_{i j}^{+}\right)^{2}\right)\right]} .
$$

Proof. According to (T1.2), the property $E\left(\tilde{P}_{+}\right)=E\left(\tilde{P}_{-}\right)=1$

implies the following:

$$
\begin{aligned}
I_{f}\left(\tilde{P}_{i}\right) & =\frac{R\left(\tilde{P}_{i}, \tilde{P}_{+}\right) / \sqrt{E\left(\tilde{P}_{i}\right) \cdot E\left(\tilde{P}_{+}\right)}}{\left(R\left(\tilde{P}_{i}, \tilde{P}_{+}\right) / \sqrt{E\left(\tilde{P}_{i}\right) \cdot E\left(\tilde{P}_{+}\right)}\right)+\left(R\left(\tilde{P}_{i}, \tilde{P}_{-}\right) / \sqrt{E\left(\tilde{P}_{i}\right) \cdot E\left(\tilde{P}_{-}\right)}\right)}=\frac{R\left(\tilde{P}_{i}, \tilde{P}_{+}\right) / \sqrt{E\left(\tilde{P}_{i}\right)}}{\left(R\left(\tilde{P}_{i}, \tilde{P}_{+}\right) / \sqrt{E\left(\tilde{P}_{i}\right)}\right)+\left(R\left(\tilde{P}_{i}, \tilde{P}_{-}\right) / \sqrt{E\left(\tilde{P}_{i}\right)}\right)} \\
& =\frac{R\left(\tilde{P}_{i}, \tilde{P}_{+}\right)}{R\left(\tilde{P}_{i}, \tilde{P}_{+}\right)+R\left(\tilde{P}_{i}, \tilde{P}_{-}\right)} .
\end{aligned}
$$


Next, using the properties in (T2.4) and (T2.5), the following results can be derived:

$$
\begin{aligned}
I_{f}\left(\tilde{P}_{i}\right)= & \frac{1}{2}\left\{\sum_{c_{j} \in C_{\mathrm{I}}}\left[w_{j} \cdot\left(\left(\mu_{i j}^{-}\right)^{2}+\left(\mu_{i j}^{+}\right)^{2}\right)\right]+\sum_{c_{j} \in C_{\mathrm{II}}}\left[w_{j} \cdot\left(\left(v_{i j}^{-}\right)^{2}+\left(v_{i j}^{+}\right)^{2}\right)\right]\right\} /\left\{\frac { 1 } { 2 } \left[\sum_{c_{j} \in C_{\mathrm{I}}}\left[w_{j} \cdot\left(\left(\mu_{i j}^{-}\right)^{2}+\left(\mu_{i j}^{+}\right)^{2}\right)\right]\right.\right. \\
& +\sum_{c_{j} \in C_{\mathrm{II}}}\left[w_{j} \cdot\left(\left(v_{i j}^{-}\right)^{2}+\left(v_{i j}^{+}\right)^{2}\right)\right]+\frac{1}{2}\left[\sum_{c_{j} \in C_{\mathrm{I}}}\left[w_{j} \cdot\left(\left(v_{i j}^{-}\right)^{2}+\left(v_{i j}^{+}\right)^{2}\right)\right]+\sum_{c_{j} \in C_{\mathrm{II}}}\left[w_{j} \cdot\left(\left(\mu_{i j}^{-}\right)^{2}+\left(\mu_{i j}^{+}\right)^{2}\right)\right]\right\} \\
= & \left\{\sum_{c_{j} \in C_{\mathrm{I}}}\left[w_{j} \cdot\left(\left(\mu_{i j}^{-}\right)^{2}+\left(\mu_{i j}^{+}\right)^{2}\right)\right]+\sum_{c_{j} \in C_{\mathrm{II}}}\left[w_{j} \cdot\left(\left(v_{i j}^{-}\right)^{2}+\left(v_{i j}^{+}\right)^{2}\right)\right]\right\} /\left\{\sum_{c_{j} \in C_{\mathrm{I}}}\left[w_{j} \cdot\left(\left(\mu_{i j}^{+}\right)^{2}+\left(v_{i j}^{+}\right)^{2}+\left(\mu_{i j}^{-}\right)^{2}+\left(v_{i j}^{-}\right)^{2}\right)\right]\right. \\
& \left.+\sum_{c_{j} \in C_{\mathrm{II}}}\left[w_{j} \cdot\left(\left(\mu_{i j}^{+}\right)^{2}+\left(v_{i j}^{+}\right)^{2}+\left(\mu_{i j}^{-}\right)^{2}+\left(v_{i j}^{-}\right)^{2}\right)\right]\right\} \\
= & \frac{\sum_{c_{j} \in C_{\mathrm{I}}}\left[w_{j} \cdot\left(\left(\mu_{i j}^{-}\right)^{2}+\left(\mu_{i j}^{+}\right)^{2}\right)\right]+\sum_{c_{j} \in C_{\mathrm{II}}}\left[w_{j} \cdot\left(\left(v_{i j}^{-}\right)^{2}+\left(v_{i j}^{+}\right)^{2}\right)\right]}{\sum_{j=1}^{n}\left[w_{j} \cdot\left(\left(\mu_{i j}^{+}\right)^{2}+\left(v_{i j}^{+}\right)^{2}+\left(\mu_{i j}^{-}\right)^{2}+\left(v_{i j}^{-}\right)^{2}\right)\right]} \\
= & \frac{\sum_{c_{j} \in C_{\mathrm{I}}}\left[w_{j} \cdot\left(\left(\mu_{i j}^{-}\right)^{2}+\left(\mu_{i j}^{+}\right)^{2}\right)\right]+\sum_{c_{j} \in C_{\mathrm{II}}}\left[w_{j} \cdot\left(\left(v_{i j}^{-}\right)^{2}+\left(v_{i j}^{+}\right)^{2}\right)\right]}{\sum_{j=1}^{n}\left[w_{j} \cdot\left(2-\left(\pi_{i j}^{-}\right)^{2}-\left(\pi_{i j}^{+}\right)^{2}\right)\right]}
\end{aligned}
$$

where $\left(\mu_{i j}^{+}\right)^{2}+\left(v_{i j}^{+}\right)^{2}=1-\left(\pi_{i j}^{-}\right)^{2}$ and $\left(\mu_{i j}^{-}\right)^{2}+\left(v_{i j}^{-}\right)^{2}=1-$ $\left(\pi_{i j}^{+}\right)^{2}$ for all $c_{j} \in C$ (i.e., $j \in\{1,2, \cdots, n\}$ ). This completes the proof.

Theorem 6. The correlation-based closeness index $I_{f}\left(\tilde{P}_{i}\right)$ satisfies the following properties:

(T6.1) $0 \leq I_{f}\left(\tilde{P}_{i}\right) \leq 1$

(T6.2) $I_{f}\left(\tilde{P}_{-}\right)=0$

(T6.3) $I_{f}\left(\tilde{P}_{+}\right)=1$
(T6.4) $I_{f}\left(\tilde{P}_{i}\right)=0$ if and only if $w_{j} \cdot \mu_{i j}^{-}=w_{j} \cdot \mu_{i j}^{+}=0$ for all $c_{j} \in C_{\mathrm{I}}$ and $w_{j} \cdot v_{i j}^{-}=w_{j} \cdot v_{i j}^{+}=0$ for all $c_{j} \in C_{\mathrm{II}}$

(T6.5) $I_{f}\left(\tilde{P}_{i}\right)=1$ if and only if $w_{j} \cdot v_{i j}^{-}=w_{j} \cdot v_{i j}^{+}=0$ for all $c_{j} \in C_{\mathrm{I}}$ and $w_{j} \cdot \mu_{i j}^{-}=w_{j} \cdot \mu_{i j}^{+}=0$ for all $c_{j} \in C_{\mathrm{II}}$

Proof. (T6.1) can be inferred directly because $K\left(\tilde{P}_{i}, \tilde{P}_{+}\right) \geq 0$, $K\left(\tilde{P}_{i}, \tilde{P}_{-}\right) \geq 0$, and $K\left(\tilde{P}_{i}, \tilde{P}_{+}\right) \leq K\left(\tilde{P}_{i}, \tilde{P}_{+}\right)+K\left(\tilde{P}_{i}, \tilde{P}_{-}\right)$.

(T6.2) From Definition 6, it is known that $\mu_{-j}^{-}=\mu_{-j}^{+}=0$ for each $c_{j} \in C_{\mathrm{I}}$ and $v_{-j}^{-}=v_{-j}^{+}=0$ for each $c_{j} \in C_{\mathrm{II}}$. From Definition 9, (T1.2), and (T2.4), the following is clear:

$$
K\left(\tilde{P}_{-}, \tilde{P}_{+}\right)=\frac{R\left(\tilde{P}_{-}, \tilde{P}_{+}\right)}{\sqrt{E\left(\tilde{P}_{-}\right) \cdot E\left(\tilde{P}_{+}\right)}}=\frac{1 / 2\left\{\sum_{c_{j} \in C_{\mathrm{I}}}\left[w_{j} \cdot\left(\left(\mu_{-j}^{-}\right)^{2}+\left(\mu_{-j}^{+}\right)^{2}\right)\right]+\sum_{c_{j} \in C_{\mathrm{II}}}\left[w_{j} \cdot\left(\left(v_{-j}^{-}\right)^{2}+\left(v_{-j}^{+}\right)^{2}\right)\right]\right\}}{\sqrt{1 \cdot 1}}=0 .
$$


It directly follows that $I_{f}\left(\tilde{P}_{-}\right)=0$; i.e., (T6.2) is valid.

(T6.3) By using (T3.1) and (T3.3), one obtains $K\left(\tilde{P}_{+}\right.$, $\left.\tilde{P}_{-}\right)=K\left(\tilde{P}_{-}, \tilde{P}_{+}\right)$and $K\left(\tilde{P}_{+}, \tilde{P}_{+}\right)=1$, respectively. Because $K\left(\tilde{P}_{-}, \tilde{P}_{+}\right)=0$ from the previous result,

$$
I_{f}\left(\tilde{P}_{+}\right)=\frac{K\left(\tilde{P}_{+}, \tilde{P}_{+}\right)}{K\left(\tilde{P}_{+}, \tilde{P}_{+}\right)+K\left(\tilde{P}_{+}, \tilde{P}_{-}\right)}=\frac{1}{1+0}=1 . .
$$

Therefore, (T6.3) is valid.

(T6.4) For the necessity, if $I_{f}\left(\tilde{P}_{i}\right)=0$, the condition of $K$ $\left(\tilde{P}_{i}, \tilde{P}_{+}\right)=0$ must be satisfied. It follows that $R\left(\tilde{P}_{i}, \tilde{P}_{+}\right)=0$ because $K\left(\tilde{P}_{i}, \tilde{P}_{+}\right)=R\left(\tilde{P}_{i}, \tilde{P}_{+}\right) / \sqrt{E\left(\tilde{P}_{i}\right) \cdot E\left(\tilde{P}_{+}\right)}$. By using the property in (T2.4), one obtains $w_{j} \cdot\left(\left(\mu_{i j}^{-}\right)^{2}+\left(\mu_{i j}^{+}\right)^{2}\right)=0$ for all $c_{j} \in C_{\mathrm{I}}$, and $w_{j} \cdot\left(\left(v_{i j}^{-}\right)^{2}+\left(v_{i j}^{+}\right)^{2}\right)=0$ for all $c_{j} \in C_{\mathrm{II}}$. Thus, the weighted membership degrees $w_{j} \cdot \mu_{i j}^{-}=w_{j} \cdot \mu_{i j}^{+}=0$ for all $c_{j} \in C_{\mathrm{I}}$, and the weighted nonmembership degrees $w_{j} \cdot v_{i j}^{-}=$ $w_{j} \cdot v_{i j}^{+}=0$ for all $c_{j} \in C_{\mathrm{II}}$. For the sufficiency, the assumptions of $w_{j} \cdot \mu_{i j}^{-}=w_{j} \cdot \mu_{i j}^{+}=0$ for $c_{j} \in C_{\mathrm{I}}$ and $w_{j} \cdot v_{i j}^{-}=w_{j} \cdot v_{i j}^{+}=0$ for $c_{j} \in C_{\text {II }}$ result in $R\left(\tilde{P}_{i}, \tilde{P}_{+}\right)=0$ and $K\left(\tilde{P}_{i}, \tilde{P}_{+}\right)=0$. Accordingly, (T6.4) is correct.

(T6.5) For the necessity, if $I_{f}\left(\tilde{P}_{i}\right)=1$, applying Theorem 5 , the following result can be acquired:

$$
\begin{aligned}
\sum_{c_{j} \in C_{\mathrm{I}}}\left[w_{j}\right. & \left.\cdot\left(\left(\mu_{i j}^{-}\right)^{2}+\left(\mu_{i j}^{+}\right)^{2}\right)\right]+\sum_{c_{j} \in C_{\mathrm{II}}}\left[w_{j} \cdot\left(\left(v_{i j}^{-}\right)^{2}+\left(v_{i j}^{+}\right)^{2}\right)\right] \\
= & \sum_{j=1}^{n}\left[w_{j} \cdot\left(2-\left(\pi_{i j}^{-}\right)^{2}-\left(\pi_{i j}^{+}\right)^{2}\right)\right]=\sum_{j=1}^{n}\left[w _ { j } \cdot \left(\left(\mu_{i j}^{-}\right)^{2}\right.\right. \\
& \left.\left.+\left(\mu_{i j}^{+}\right)^{2}+\left(v_{i j}^{-}\right)^{2}+\left(v_{i j}^{+}\right)^{2}\right)\right]=\sum_{c_{j} \in C_{\mathrm{I}}}\left[w _ { j } \cdot \left(\left(\mu_{i j}^{-}\right)^{2}\right.\right. \\
& \left.\left.+\left(\mu_{i j}^{+}\right)^{2}+\left(v_{i j}^{-}\right)^{2}+\left(v_{i j}^{+}\right)^{2}\right)\right]+\sum_{c_{j} \in C_{\mathrm{II}}}\left[w _ { j } \cdot \left(\left(\mu_{i j}^{-}\right)^{2}\right.\right. \\
& \left.\left.+\left(\mu_{i j}^{+}\right)^{2}+\left(v_{i j}^{-}\right)^{2}+\left(v_{i j}^{+}\right)^{2}\right)\right] .
\end{aligned}
$$

Thus, $\quad \sum_{c_{j} \in C_{\mathrm{I}}}\left[w_{j} \cdot\left(\left(v_{i j}^{-}\right)^{2}+\left(v_{i j}^{+}\right)^{2}\right)\right]=\sum_{c_{j} \in C_{\mathrm{II}}}\left[w_{j} \cdot\left(\left(\mu_{i j}^{-}\right)^{2}+\right.\right.$ $\left.\left.\left(\mu_{i j}^{+}\right)^{2}\right)\right]=0$. Therefore, it can be concluded that the weighted nonmembership degrees $w_{j} \cdot v_{i j}^{-}=w_{j} \cdot v_{i j}^{+}=0$ for all $c_{j} \in C_{\mathrm{I}}$, and the weighted membership degrees $w_{j} \cdot \mu_{i j}^{-}=w_{j} \cdot \mu_{i j}^{+}=0$ for all $c_{j} \in C_{\mathrm{II}}$. For the sufficiency, the assumption of $w_{j}$. $v_{i j}^{-}=w_{j} \cdot v_{i j}^{+}=0$ for $c_{j} \in C_{\mathrm{I}}$ infers that $\sum_{c_{j} \in C_{\mathrm{I}}}\left[w_{j} \cdot\left(\left(\mu_{i j}^{-}\right)^{2}+\right.\right.$ $\left.\left.\left(\mu_{i j}^{+}\right)^{2}\right)\right]=\sum_{c_{j} \in C_{\mathrm{I}}}\left[w_{j} \cdot\left(\left(\mu_{i j}^{-}\right)^{2}+\left(\mu_{i j}^{+}\right)^{2}+\left(v_{i j}^{-}\right)^{2}+\left(v_{i j}^{+}\right)^{2}\right)\right]$. Analogously, one can obtain $\sum_{c_{j} \in C_{\text {II }}}\left[w_{j} \cdot\left(\left(v_{i j}^{-}\right)^{2}+\left(v_{i j}^{+}\right)^{2}\right)\right]=$ $\sum_{c_{j} \in C_{\text {II }}}\left[w_{j} \cdot\left(\left(\mu_{i j}^{-}\right)^{2}+\left(\mu_{i j}^{+}\right)^{2}+\left(v_{i j}^{-}\right)^{2}+\left(v_{i j}^{+}\right)^{2}\right)\right]$ based on the assumption that $w_{j} \cdot \mu_{i j}^{-}=w_{j} \cdot \mu_{i j}^{+}=0$ for $c_{j} \in C_{\mathrm{II}}$. Accordingly, these results yield that $\sum_{c_{j} \in C_{\mathrm{I}}}\left[w_{j} \cdot\left(\left(\mu_{i j}^{-}\right)^{2}+\left(\mu_{i j}^{+}\right)^{2}\right)\right]+$ $\sum_{c_{j} \in C_{\mathrm{II}}}\left[w_{j} \cdot\left(\left(v_{i j}^{-}\right)^{2}+\left(v_{i j}^{+}\right)^{2}\right)\right]=\sum_{j=1}^{n}\left[w_{j} \cdot\left(2-\left(\pi_{i j}^{-}\right)^{2}-\left(\pi_{i j}^{+}\right)^{2}\right)\right]$.
$I_{f}\left(\tilde{P}_{i}\right)=1$ by Theorem 5 . Hence, (T6.5) is valid, which completes the proof.

Based on the useful and desirable properties proved in the previous theorems, the developed correlation-based closeness indices $I_{d}\left(\tilde{P}_{i}\right)$ and $I_{f}\left(\tilde{P}_{i}\right)$ can assist decision-makers in determining the ultimate priority orders among candidate alternatives. Notably, the larger the $I_{d}\left(\tilde{P}_{i}\right)$ (or $\left.I_{f}\left(\tilde{P}_{i}\right)\right)$ value is, the greater the preference is for the IVPF characteristics $\tilde{P}_{i}$. More specifically, the condition $I_{d}\left(\tilde{P}_{i}\right)>I_{d}\left(\tilde{P}_{i^{\prime}}\right)\left(\right.$ or $\left.I_{f}\left(\tilde{P}_{i}\right)>I_{f}\left(\tilde{P}_{i^{\prime}}\right)\right)$ indicates that $\tilde{P}_{i}$ is better than $\tilde{P}_{i^{\prime}}$ or that $z_{i}$ is preferred to $z_{i^{\prime}}$; alternatively, $I_{d}\left(\tilde{P}_{i}\right)<I_{d}\left(\tilde{P}_{i^{\prime}}\right) \quad\left(\right.$ or $\left.I_{f}\left(\tilde{P}_{i}\right)<I_{f}\left(\tilde{P}_{i^{\prime}}\right)\right)$ indicates that $\tilde{P}_{i}$ is worse than $\tilde{P}_{i^{\prime}}$ or that $z_{i}$ is less preferred to $z_{i^{\prime}}$. The condition $I_{d}\left(\tilde{P}_{i}\right)=I_{d}\left(\tilde{P}_{i^{\prime}}\right)$ (or $\left.I_{f}\left(\tilde{P}_{i}\right)=I_{f}\left(\tilde{P}_{i^{\prime}}\right)\right)$ implies indifference between $\tilde{P}_{i}$ and $\tilde{P}_{i^{\prime}}$ or equal preference between $z_{i}$ and $z_{i^{\prime}}$. Following such a ranking procedure, this paper establishes a novel IVPF compromise approach using correlation-based closeness indices based on information energies and correlations in the IVPF context. Employing the proposed approach and techniques to address an MCDA problem within the IVPF environment, the ultimate priority orders among candidate alternatives can be effectively determined according to the descending order of the $I_{d}\left(\tilde{P}_{i}\right)$ and $I_{f}\left(\tilde{P}_{i}\right)$ values when underlying anchored judgments with displaced and fixed ideals, respectively.

3.3. Proposed Algorithm. This subsection intends to provide useful and effective algorithmic procedures for implementing the developed IVPF compromise approaches using correlation-based closeness indices with respect to the displaced/fixed ideal IVPF solutions. First, in the case of anchored judgments with displaced ideals, the proposed IVPF compromise approach for solving MCDA problems under complex uncertainty based on IVPF sets can be summarized as the following algorithmic procedure.

Concerning anchored judgments with fixed ideals, the algorithmic procedure can be significantly simplified because of desirable and valuable properties proved in Theorems 5 and 6 . Based on the correlation-based closeness indices with respect to the anchor points (i.e., $([1,1],[0,0])$ and $([0,0],[1$, 1])) of fixed ideals, the proposed IVPF compromise approach for managing MCDA problems within the IVPF environment is summarized below.

\section{Practical Application with Comparative Discussions}

This section utilizes a practical MCDA problem of bridge construction methods in Taiwan to examine the usefulness and effectiveness of the proposed IVPF compromise approach. Furthermore, this section investigates the application results yielded by the developed Algorithms 1 and 2 to make a thorough inquiry about practical implications.

4.1. Problem Statement of a Case Study. Taiwan is characterized by a diversity of landscapes that are rugged and complex 
Step I.1: formulate an MCDA problem within the IVPF environment. Specify the set of candidate alternatives $Z=\left\{z_{1}, z_{2}, \cdots, z_{m}\right\}$ and the set of evaluative criteria $C=\left\{c_{1}, c_{2}, \cdots, c_{n}\right\}$, which is divided into $C_{\mathrm{I}}$ and $C_{\mathrm{II}}$.

Step I.2: establish the weight vector $\mathrm{w}^{T}=\left(w_{1}, w_{2}, \cdots, w_{n}\right)^{T}$ with respect to $n$ criteria. Identify the IVPF evaluative rating $\tilde{p}_{i j}$ of each alternative $z_{i} \in Z$ in relation to criterion $c_{j} \in C$. Construct an IVPF decision matrix $\tilde{\mathrm{p}}=\left[\tilde{p}_{i j}\right]_{m \times n}$.

Step I.3: identify the characteristics $\tilde{P}_{*}$ of the displaced positive-ideal IVPF solution $z_{*}$ by means of (13) and (15). In addition, determine the characteristics $\tilde{P}_{\#}$ of the displaced negative-ideal IVPF solution $z_{\#}$ using (14) and (16).

Step I.4: use (19) to compute the information energy $E\left(\tilde{P}_{i}\right)$ of the IVPF characteristics $\tilde{P}_{i}$ for each $z_{i} \in Z$ in $\tilde{\mathrm{p}}$. In addition, derive $E\left(\tilde{P}_{*}\right)$ and $E\left(\tilde{P}_{\#}\right)$ for the displaced ideal IVPF solutions $z_{*}$ and $z_{\#}$.

Step I.5: Derive the correlations $R\left(\tilde{P}_{i}, \tilde{P}_{*}\right)$ and $R\left(\tilde{P}_{i}, \tilde{P}_{\#}\right)$ between $\tilde{P}_{i}$ and $\tilde{P}_{*}$ and between $\tilde{P}_{i}$ and $\tilde{P}_{\#}$, respectively, using (20).

Step I.6: employ (21) to calculate the correlation coefficients $K\left(\tilde{P}_{i}, \tilde{P}_{*}\right)$ and $K\left(\tilde{P}_{i}, \tilde{P}_{\#}\right)$ for each IVPF characteristics $\tilde{P}_{i}$ in $\tilde{\mathrm{p}}$.

Step I.7: determine the correlation-based closeness index $I_{d}\left(\tilde{P}_{i}\right)$ using (24) for each $z_{i} \in Z$.

Step I.8: rank the $m$ alternatives in accordance with the $I_{d}\left(\tilde{P}_{i}\right)$ values. The alternative with the largest $I_{d}\left(\tilde{P}_{i}\right)$ value is the best choice.

Algorithm 1: For anchored judgments with displaced ideals.

Steps II.1 and II.2: see Steps I.1 and I.2 of Algorithm 1.

Step II.3: apply (26) to calculate the correlation-based closeness index $I_{f}\left(\tilde{P}_{i}\right)$ of the IVPF characteristics $\tilde{P}_{i}$ for each $z_{i} \in Z$ in $\tilde{\mathrm{p}}$.

Step II.4: rank the $m$ alternatives in accordance with the $I_{f}\left(\tilde{P}_{i}\right)$ values. The alternative with the largest $I_{f}\left(\tilde{P}_{i}\right)$ value is the best choice.

Algorithm 2: For anchored judgments with fixed ideals.

and feature different terrain characteristics (e.g., basins and mountains). Because Taiwan features numerous mountains and rivers, the implementation of transportation projects requires bridges to join disconnected regions. However, due to intensive domestic land development, increasing land costs, and various residential environment factors and to avoid considerable demolitions and reduce negative environmental impact, transportation projects have generally occurred on hills and in mountain areas. This relatively unfavorable geology makes bridge-site selection procedures particularly complex. In urban areas, flyovers and viaducts are used to avoid road traffic congestion. These endeavors have subsequently increased the number of bridge projects in proportion to overall transportation projects.

Earthquakes, flooding, and typhoons are natural disasters that often occur in Taiwan because of Taiwan's location on the west side of the Ring of Fire at the junction of the Philippine Sea Plate and the Eurasian Plate. These disaster events, combined with rapid changes in the global climate and subsequent changes in related areas such as environmental and climate conditions, have exposed bridges to an increasing number of natural threats. Furthermore, as the length of bridges increases, aesthetic considerations and the demand to join or integrate multiple bridges together arise, inflaming the problems of labor shortage and growing wages in the construction industry. Bridge projects therefore encounter increasingly challenging construction conditions that can no longer be resolved by traditional means. Accordingly, novel, economical, and highly efficient bridge construction methods that can overcome terrain constraints and sustain attacks from natural disasters are in great demand for construction projects.
Hualien and Taitung have some of Taiwan's most famous tourist attractions; however, they are also the regions that are most prone to natural disasters. The Suhua Highway is the main road connecting Taitung, Hualien, and the Taipei metropolitan area. Nevertheless, because the Suhua Highway is meandering and steep, vehicle accidents regularly occur. In poor weather conditions such as strong winds, rains, and typhoons, disasters frequently occur, resulting in the Suhua Highway being blocked or bridges collapsing, in turn creating service interruptions. To address this problem, the central government proposed the Suhua Highway Alternative Road Project, in which safe and fast alternative roads were developed for the disaster-prone sections of the Suhua Highway.

This study investigated the construction of the concretebased bridge superstructure for the Suhua Highway Alternative Road Project, which involved four widely used bridge construction methods: the advanced shoring method, the incremental launching method, the balanced cantilever method, and the precast segmental method. First, the advanced shoring method creates relatively negligible pollution and has a relatively minimal environmental impact. It is suitable for the site in which the project was performed and is not affected by terrain constraints. However, the method entails relatively high damage costs and creates less durable bridges. Second, the incremental launching method allows the reuse of construction equipment, reducing construction costs. It also has a smaller effect on traffic during construction. However, it compromises the aesthetics of the surrounding scenery. Third, the balanced cantilever method enables easy control over construction quality, facilitating longer bridge life and reducing bridge maintenance and repairs. However, the method is relatively expensive and 
can be delayed or otherwise affected by poor weather conditions. Fourth, the precast segmental method has a relatively negligible effect on the surrounding landscape and existing traffic conditions during construction. However, it is limited by the climate and geological conditions of the site.

The bridge construction criteria selected for this project were based on those proposed by Pan [58] and Malekly et al. [21], which are explained as follows: (i) durability: the service life of the bridge built using a given method; the bridge should have a permanent service life and not require frequent maintenance; (ii) damage cost: the maintenance and repair costs of the bridge when damaged; (iii) construction cost: the overall costs involved in the construction of the bridge, from the start of the design process to the completion of the final construction; (iv) traffic effect: the negative effect of the bridge construction process on existing traffic; (v) site condition: the conditions of the site, which includes the suitability of the construction method to a site's terrain structure and whether the site imposes constraints on the construction methods; (vi) climatic condition: whether the climate of the site accelerates the damage inflicted on the bridge or delays its construction; (vii) landscape: whether the bridge has a negative effect on the landscape of the surrounding environment, or whether the bridge itself is aesthetically attractive; and (viii) environmental impact: damage caused by the construction method and process to the surrounding environment. Of the eight criteria, durability and site condition are benefit criteria, whereas the remaining criteria are cost criteria.

\subsection{Application of Selecting a Suitable Bridge Construction} Method. The practical case concerning the selection problem of bridge construction methods is modified from the case introduced by Chen $[18,42,59,60]$ and Wang and Chen [52]. This case involves an MCDA problem of how to select the most suitable bridge construction method for the Suhua Highway Alternative Road Project in the Hualien and Taitung areas of Taiwan. As explained earlier, this MCDA problem is defined by four candidate bridge-construction methods and eight criteria for evaluating the alternatives.

First, the developed methodology with Algorithm 1 was utilized to help the authority select the most appropriate bridge construction method. In Step I.1, the set of candidate alternatives is denoted by $Z=\left\{z_{1}\right.$ (the advanced shoring method), $z_{2}$ (the incremental launching method), $z_{3}$ (the balanced cantilever method), and $z_{4}$ (the precast segmental method) $\}$. The set of evaluative criteria is denoted by $C=$ $\left\{c_{1}\right.$ (durability), $c_{2}$ (damage cost), $c_{3}$ (construction cost), $c_{4}$ (traffic effect), $c_{5}$ (site condition), $c_{6}$ (climatic condition), $c_{7}$ (landscape), and $c_{8}$ (environmental impact) $\}$, which is divided into $C_{\mathrm{I}}=\left\{c_{1}, c_{5}\right\}$ and $C_{\mathrm{II}}=\left\{c_{2}, c_{3}, c_{4}, c_{6}, c_{7}, c_{8}\right\}$.

The original evaluative ratings data presented in Chen $[18,59,60]$ and Wang and Chen [52] belong to Atanassov's interval-valued intuitionistic fuzzy (IVIF) sets [61], not IVPF sets. To validate the feasibility of the IVPF outranking method with an assignment model, Chen [42] converted the IVIF evaluative ratings into the IVPF values by simply recalculating the corresponding intervals of the indeterminacy degree. That is, Chen did not adjust interval-valued degrees of membership and nonmembership with respect to each original IVIF evaluative rating. Nevertheless, although there can be no doubt that the transformed data belong to IVPF sets, there must be considerable doubt concerning the distinct interrelationships among membership, nonmembership, and indeterminacy degrees. In contrast with Chen [42], this paper proposes another approach to reasonably convert the IVIF data into the IVPF values for conducting a substantive transformation of the IVPF representation and maintaining essential information conveyed by the original IVIF evaluative ratings. More specifically, let $\tilde{p}_{i j}^{0}$ denote the IVIF evaluative rating of an alternative $z_{i} \in Z$ in terms of criterion $c_{j} \in C$ within the IVIF decision environment, where $\tilde{p}_{i j}^{0}=\left(\left[\mu_{i j}^{0-}\right.\right.$, $\left.\left.\mu_{i j}^{0+}\right],\left[v_{i j}^{0-}, v_{i j}^{0+}\right]\right)$ and the interval of the indeterminacy degree $\pi_{i j}^{0}=\left[\pi_{i j}^{0-}, \pi_{i j}^{0+}\right]=\left[1-\mu_{i j}^{0+}-v_{i j}^{0+}, 1-\mu_{i j}^{0-}-v_{i j}^{0-}\right]$. To adapt the $\tilde{p}_{i j}^{0}$ value to the IVPF environment, the IVPF evaluative rating $\tilde{p}_{i j}$ related to $\tilde{p}_{i j}^{0}$ can be determined in the following manner:

$$
\begin{aligned}
& \tilde{p}_{i j}=\left(\left[\mu_{i j}^{-}, \mu_{i j}^{+}\right],\left[v_{i j}^{-}, v_{i j}^{+}\right]\right) \\
& =\left(\left[\operatorname { m i n } \left\{\sqrt{\frac{\left(\mu_{i j}^{0-}\right)^{2}}{\left(\mu_{i j}^{0-}\right)^{2}+\left(v_{i j}^{0-}\right)^{2}+\left(\pi_{i j}^{0+}\right)^{2}}},\right.\right.\right. \\
& \left.\sqrt{\frac{\left(\mu_{i j}^{0+}\right)^{2}}{\left(\mu_{i j}^{0+}\right)^{2}+\left(\nu_{i j}^{0+}\right)^{2}+\left(\pi_{i j}^{0-}\right)^{2}}}\right\}, \\
& \max \left\{\sqrt{\frac{\left(\mu_{i j}^{0-}\right)^{2}}{\left(\mu_{i j}^{0-}\right)^{2}+\left(\nu_{i j}^{0-}\right)^{2}+\left(\pi_{i j}^{0+}\right)^{2}}},\right. \\
& \left.\sqrt{\frac{\left(\mu_{i j}^{0+}\right)^{2}}{\left(\mu_{i j}^{0+}\right)^{2}+\left(v_{i j}^{0+}\right)^{2}+\left(\pi_{i j}^{0-}\right)^{2}}}\right\}, \\
& {\left[\operatorname { m i n } \left\{\sqrt{\frac{\left(v_{i j}^{0-}\right)^{2}}{\left(\mu_{i j}^{0-}\right)^{2}+\left(v_{i j}^{0-}\right)^{2}+\left(\pi_{i j}^{0+}\right)^{2}}},\right.\right.} \\
& \left.\sqrt{\frac{\left(v_{i j}^{0+}\right)^{2}}{\left(\mu_{i j}^{0+}\right)^{2}+\left(\nu_{i j}^{0+}\right)^{2}+\left(\pi_{i j}^{0-}\right)^{2}}}\right\} \text {, } \\
& \max \left\{\sqrt{\frac{\left(v_{i j}^{0-}\right)^{2}}{\left(\mu_{i j}^{0-}\right)^{2}+\left(v_{i j}^{0-}\right)^{2}+\left(\pi_{i j}^{0+}\right)^{2}}},\right. \\
& \left.\left.\sqrt{\frac{\left(v_{i j}^{0+}\right)^{2}}{\left(\mu_{i j}^{0+}\right)^{2}+\left(v_{i j}^{0+}\right)^{2}+\left(\pi_{i j}^{0-}\right)^{2}}}\right\}\right],
\end{aligned}
$$


where $0 \leq \mu_{i j}^{-} \leq \mu_{i j}^{+} \leq 1,0 \leq v_{i j}^{-} \leq v_{i j}^{+} \leq 1$, and $\left(\mu_{i j}^{+}\right)^{2}+\left(v_{i j}^{+}\right)^{2} \leq 1$. The interval of the indeterminacy degree corresponding to $\tilde{p}_{i j}$ can be computed using (8) or, equivalently, be determined as follows:

$$
\begin{aligned}
& \pi_{i j}=\left[\pi_{i j}^{-}, \pi_{i j}^{+}\right]=\left[\operatorname { m i n } \left\{\sqrt{\frac{\left(\pi_{i j}^{0-}\right)^{2}}{\left(\mu_{i j}^{0+}\right)^{2}+\left(v_{i j}^{0+}\right)^{2}+\left(\pi_{i j}^{0-}\right)^{2}}},\right.\right. \\
& \left.\sqrt{\frac{\left(\pi_{i j}^{0+}\right)^{2}}{\left(\mu_{i j}^{0-}\right)^{2}+\left(v_{i j}^{0-}\right)^{2}+\left(\pi_{i j}^{0+}\right)^{2}}}\right\} \text {, } \\
& \max \left\{\sqrt{\frac{\left(\pi_{i j}^{0-}\right)^{2}}{\left(\mu_{i j}^{0+}\right)^{2}+\left(v_{i j}^{0+}\right)^{2}+\left(\pi_{i j}^{0-}\right)^{2}}},\right. \\
& \left.\sqrt{\frac{\left(\pi_{i j}^{0+}\right)^{2}}{\left(\mu_{i j}^{0-}\right)^{2}+\left(v_{i j}^{0-}\right)^{2}+\left(\pi_{i j}^{0+}\right)^{2}}}\right\} \text {, }
\end{aligned}
$$

where $0 \leq \pi_{i j}^{-} \leq \pi_{i j}^{+} \leq 1,\left(\mu_{i j}^{-}\right)^{2}+\left(v_{i j}^{-}\right)^{2}+\left(\pi_{i j}^{+}\right)^{2}=1$, and $\left(\mu_{i j}^{+}\right)^{2}+$ $\left(v_{i j}^{+}\right)^{2}+\left(\pi_{i j}^{-}\right)^{2}=1$. The IVPF evaluative ratings required in the bridge construction case can then be acquired according to the transformation procedure in (32) and (33).

Table 1 contrasts the original IVIF data with the obtained IVPF results. Based on the $\tilde{p}_{i j}$ values in this table, the IVPF decision matrix $\tilde{\mathrm{p}}=\left[\tilde{p}_{i j}\right]_{4 \times 8}$, along with the IVPF characteristics $\tilde{P}_{i}$, can be obtained correspondingly. Consider the IVIF evaluative rating $\tilde{p}_{37}^{0}=([0.18,0.19],[0.68,0.74])$ with $\pi_{37}^{0}=$ $[0.07,0.14]$ as an example. Employing (32) and (33), $\tilde{p}_{37}$ and $\pi_{37}$ were calculated as follows:

$$
\begin{aligned}
\tilde{p}_{37}= & \left(\left[\operatorname { m i n } \left\{\sqrt{\frac{(0.18)^{2}}{(0.18)^{2}+(0.68)^{2}+(0.14)^{2}}},\right.\right.\right. \\
& \sqrt{\left.\frac{(0.19)^{2}}{(0.19)^{2}+(0.74)^{2}+(0.07)^{2}}\right\}} \\
& \max \left\{\sqrt{\frac{(0.18)^{2}}{(0.18)^{2}+(0.68)^{2}+(0.14)^{2}}}\right. \\
& \sqrt{\left.\frac{(0.19)^{2}}{(0.19)^{2}+(0.74)^{2}+(0.07)^{2}}\right\}}, \\
& \sqrt{\left.\frac{\min \left\{\sqrt{\frac{(0.68)^{2}}{(0.18)^{2}+(0.68)^{2}+(0.14)^{2}}}\right.}{(0.19)^{2}+(0.74)^{2}+(0.07)^{2}}\right\}}
\end{aligned}
$$

$$
\begin{aligned}
& \max \left\{\sqrt{\frac{(0.68)^{2}}{(0.18)^{2}+(0.68)^{2}+(0.14)^{2}}},\right. \\
= & ([\min \{0.2510,0.2477\}, \max \{0.2510,0.2477\}], \\
& {[\min \{0.9481,0.9645\}, \max \{0.9481,0.9645\}])) } \\
= & ([0.2477,0.2510],[0.9481,0.9645]), \\
\pi_{37}= & {\left[\operatorname { m i n } \left\{\sqrt{\frac{(0.07)^{2}}{(0.19)^{2}+(0.74)^{2}+(0.07)^{2}},}\right.\right.} \\
& \sqrt{\left.\frac{(0.14)^{2}}{(0.18)^{2}+(0.68)^{2}+(0.14)^{2}}\right\},} \\
& \max \left\{\sqrt{\frac{(0.07)^{2}}{(0.19)^{2}+(0.74)^{2}+(0.07)^{2}},}\right. \\
& \sqrt{\left.\left.\frac{(0.14)^{2}}{(0.18)^{2}+(0.68)^{2}+(0.14)^{2}}\right\}\right]} \\
= & {[\min \{0.0912,0.1952\}, \max \{0.0912,0.1952\}] } \\
= & {[0.0912,0.1952] . }
\end{aligned}
$$

The original case concerning the selection of bridge construction methods was initiated by Chen [59], and in this case the importance weights of the criteria were expressed with a set of incomplete and inconsistent information. In other words, the importance weights of the criteria are unknown a priori. Thus, this paper adopts the preference information provided in Chen [18] and Wang and Chen [52]. Nevertheless, the criterion weights are expressed with IVIF values, which is different from the scalar weights used in the proposed methodology. To address this issue, the concept of score functions based on IVIF information was employed to determine nonfuzzy and normalized weights for each criterion. Let $\tilde{w}_{j}^{0}$ denote the IVIF importance weight of a criterion $c_{j} \in C$ within the IVIF environment, where $\tilde{w}_{j}^{0}=\left(\left[\omega_{j}^{0-}, \omega_{j}^{0+}\right],\left[\omega_{j}^{0-}, \omega_{j}^{0+}\right]\right)$. This paper applies the concept of score functions to obtain comparable values of $\tilde{w}_{j}^{0}$ and then performs a normalization of the obtained results to fulfill the normalization condition. The widely used score function of an IVIF value is between -1 and 1 [52]. To facilitate the following study, this paper attempts to employ the modified score function introduced by $\mathrm{Yu}$ et al. [62] because the range of their definition is between 0 and 1. According to Yu et al.'s definition (i.e., the score function of the IVIF value $\tilde{w}_{j}^{0}$ is defined as $(1 / 4)$. $\left.\left(2+\omega_{j}^{0-}+\omega_{j}^{0+}-\omega_{j}^{0-}-\omega_{j}^{0+}\right)\right)$, the normalized weight of criterion $c_{j}$ is determined as follows: 
TABLE 1: Evaluative ratings and indeterminacy intervals in the bridge construction case.

\begin{tabular}{|c|c|c|c|c|c|}
\hline \multirow[b]{2}{*}{$z_{i}$} & \multirow[b]{2}{*}{$c_{j}$} & \multicolumn{2}{|c|}{ Original IVIF evaluative ratings } & \multicolumn{2}{|c|}{ Converted IVPF evaluative ratings } \\
\hline & & $\tilde{p}_{i j}^{0}$ & $\pi_{i j}^{0}$ & $\tilde{p}_{i j}$ & $\pi_{i j}$ \\
\hline \multirow{8}{*}{$z_{1}$} & $c_{1}$ & $([0.28,0.35],[0.33,0.46])$ & {$[0.19,0.39]$} & $([0.4806,0.5752],[0.5664,0.7560])$ & {$[0.3123,0.6694]$} \\
\hline & $c_{2}$ & $([0.43,0.58],[0.16,0.17])$ & {$[0.25,0.41]$} & $([0.6988,0.8868],[0.2599,0.2600])$ & {$[0.3822,0.6663]$} \\
\hline & $c_{3}$ & $([0.08,0.16],[0.63,0.75])$ & {$[0.09,0.29]$} & $([0.1146,0.2072],[0.9024,0.9713])$ & {$[0.1166,0.4154]$} \\
\hline & $c_{4}$ & $([0.07,0.49],[0.38,0.41])$ & {$[0.10,0.55]$} & $([0.1041,0.7577],[0.5653,0.6340])$ & {$[0.1546,0.8183]$} \\
\hline & $c_{5}$ & $([0.64,0.67],[0.15,0.33])$ & {$[0.00,0.21]$} & $([0.8971,0.9274],[0.2174,0.4418])$ & {$[0.0000,0.3043]$} \\
\hline & $c_{6}$ & $([0.07,0.14],[0.64,0.74])$ & {$[0.12,0.29]$} & $([0.0991,0.1836],[0.9064,0.9703])$ & {$[0.1574,0.4107]$} \\
\hline & $c_{7}$ & $([0.14,0.21],[0.34,0.37])$ & {$[0.42,0.52]$} & $([0.2198,0.3513],[0.5339,0.6189])$ & {$[0.7025,0.8165]$} \\
\hline & $c_{8}$ & $([0.04,0.09],[0.88,0.90])$ & {$[0.01,0.08]$} & $([0.0452,0.0995],[0.9949,0.9950])$ & {$[0.0111,0.0904]$} \\
\hline \multirow{8}{*}{$z_{2}$} & $c_{1}$ & $([0.68,0.71],[0.06,0.26])$ & {$[0.03,0.26]$} & $([0.9309,0.9383],[0.0821,0.3436])$ & {$[0.0396,0.3559]$} \\
\hline & $c_{2}$ & $([0.04,0.12],[0.61,0.86])$ & {$[0.02,0.35]$} & $([0.0568,0.1382],[0.8660,0.9901])$ & {$[0.0230,0.4969]$} \\
\hline & $c_{3}$ & $([0.09,0.26],[0.33,0.46])$ & {$[0.28,0.58]$} & $([0.1337,0.4348],[0.4901,0.7692])$ & {$[0.4682,0.8614]$} \\
\hline & $c_{4}$ & $([0.12,0.23],[0.64,0.67])$ & {$[0.10,0.24]$} & $([0.1729,0.3215],[0.9222,0.9365])$ & {$[0.1398,0.3458]$} \\
\hline & $c_{5}$ & $([0.37,0.39],[0.26,0.29])$ & {$[0.32,0.37]$} & $([0.6332,0.6702],[0.4450,0.4984])$ & {$[0.5499,0.6332]$} \\
\hline & $c_{6}$ & $([0.18,0.19],[0.74,0.78])$ & {$[0.03,0.08]$} & $([0.2351,0.2365],[0.9664,0.9709])$ & {$[0.0373,0.1045]$} \\
\hline & $c_{7}$ & $([0.49,0.66],[0.18,0.26])$ & {$[0.08,0.33]$} & $([0.7934,0.9245],[0.2915,0.3642])$ & {$[0.1121,0.5343]$} \\
\hline & $c_{8}$ & $([0.18,0.41],[0.17,0.28])$ & {$[0.31,0.65]$} & $([0.2588,0.7005],[0.2444,0.4784])$ & {$[0.5296,0.9345]$} \\
\hline \multirow{8}{*}{$z_{3}$} & $c_{1}$ & $([0.72,0.77],[0.17,0.20])$ & {$[0.03,0.11]$} & $([0.9627,0.9672],[0.2273,0.2512])$ & {$[0.0377,0.1471]$} \\
\hline & $c_{2}$ & $([0.03,0.07],[0.66,0.76])$ & {$[0.17,0.31]$} & $([0.0411,0.0895],[0.9044,0.9720])$ & {$[0.2174,0.4248]$} \\
\hline & $c_{3}$ & $([0.05,0.18],[0.36,0.63])$ & {$[0.19,0.59]$} & $([0.0722,0.2639],[0.5195,0.9235])$ & {$[0.2785,0.8514]$} \\
\hline & $c_{4}$ & $([0.35,0.45],[0.39,0.44])$ & {$[0.11,0.26]$} & $([0.5983,0.7043],[0.6667,0.6887])$ & {$[0.1722,0.4445]$} \\
\hline & $c_{5}$ & $([0.64,0.67],[0.15,0.33])$ & {$[0.00,0.21]$} & $([0.8971,0.9274],[0.2174,0.4418])$ & {$[0.0000,0.3043]$} \\
\hline & $c_{6}$ & $([0.14,0.36],[0.22,0.40])$ & {$[0.24,0.64]$} & $([0.2026,0.6110],[0.3183,0.6788])$ & {$[0.4073,0.9261]$} \\
\hline & $c_{7}$ & $([0.18,0.19],[0.68,0.74])$ & {$[0.07,0.14]$} & $([0.2477,0.2510],[0.9481,0.9645])$ & {$[0.0912,0.1952]$} \\
\hline & $c_{8}$ & $([0.36,0.40],[0.44,0.58])$ & {$[0.02,0.20]$} & $([0.5675,0.5974],[0.7301,0.8229])$ & {$[0.0284,0.3319]$} \\
\hline \multirow{8}{*}{$z_{4}$} & $c_{1}$ & $([0.37,0.52],[0.33,0.41])$ & {$[0.07,0.30]$} & $([0.6385,0.7809],[0.5695,0.6157])$ & {$[0.1051,0.5177]$} \\
\hline & $c_{2}$ & $([0.26,0.36],[0.46,0.64])$ & {$[0.00,0.28]$} & $([0.4348,0.4903],[0.7692,0.8716])$ & {$[0.0000,0.4682]$} \\
\hline & $c_{3}$ & $([0.36,0.40],[0.52,0.52])$ & {$[0.08,0.12]$} & $([0.5592,0.6052],[0.7868,0.8078])$ & {$[0.1210,0.1864]$} \\
\hline & $c_{4}$ & $([0.17,0.40],[0.29,0.48])$ & {$[0.12,0.54]$} & $([0.2673,0.6287],[0.4559,0.7544])$ & {$[0.1886,0.8489]$} \\
\hline & $c_{5}$ & $([0.15,0.31],[0.21,0.62])$ & {$[0.07,0.64]$} & $([0.2174,0.4450],[0.3043,0.8899])$ & {$[0.1005,0.9274]$} \\
\hline & $c_{6}$ & $([0.26,0.85],[0.13,0.14])$ & {$[0.01,0.61]$} & $([0.3848,0.9866],[0.1625,0.1924])$ & {$[0.0116,0.9027]$} \\
\hline & $c_{7}$ & $([0.04,0.15],[0.83,0.84])$ & {$[0.01,0.13]$} & $([0.0476,0.1758],[0.9844,0.9868])$ & {$[0.0117,0.1546]$} \\
\hline & $c_{8}$ & $([0.13,0.21],[0.75,0.77])$ & {$[0.02,0.12]$} & $([0.1687,0.2630],[0.9645,0.9733])$ & {$[0.0251,0.1557]$} \\
\hline
\end{tabular}

$$
w_{j}=\frac{2+\omega_{j}^{0-}+\omega_{j}^{0+}-\omega_{j}^{0-}-\omega_{j}^{0+}}{\sum_{j^{\prime}=1}^{n}\left(2+\omega_{j^{\prime}}^{0-}+\omega_{j^{\prime}}^{0+}-\omega_{j^{\prime}}^{0-}-\omega_{j^{\prime}}^{0+}\right)},
$$

where $0 \leq w_{j} \leq 1$ and $\sum_{j=1}^{n} w_{j}=1$. Considering the bridge construction case presented in Chen [18] and Wang and Chen [52], the data of the IVIF importance weights are given as follows: $\tilde{w}_{1}^{0}=([0.49,0.67],[0.08,0.12]), \tilde{w}_{2}^{0}=([0.30,0.64]$, $[0.09,0.21]), \quad \tilde{w}_{3}^{0}=([0.26,0.54],[0.18,0.32]), \quad \tilde{w}_{4}^{0}=([0.11$, $0.33],[0.24,0.43]), \tilde{w}_{5}^{0}=([0.42,0.56],[0.04,0.07]), \tilde{w}_{6}^{0}=([0.38$,
$0.54],[0.04,0.24]), \tilde{w}_{7}^{0}=([0.55,0.69],[0.09,0.18])$, and $\tilde{w}_{8}^{0}=$ $([0.54,0.63],[0.10,0.13])$. Using (35), the obtained results of the nonfuzzy and normalized weights are as follows: $w_{1}=$ $0.1404, w_{2}=0.1252, w_{3}=0.1090, w_{4}=0.0839, w_{5}=0.1361$, $w_{6}=0.1252, w_{7}=0.1408$, and $w_{8}=0.1394$. The weight vector $\mathrm{w}^{T}=\left(w_{1}, w_{2}, \cdots, w_{8}\right)^{T}$ can be acquired correspondingly.

In Step I.3, applying (15) and (16), the characteristics $\tilde{P}_{*}$ and $\tilde{P}_{\#}$ of the displaced ideal IVPF solutions $z_{*}$ and $z_{\# \text {, }}$, respectively, were identified based on the $\tilde{p}_{i j}$ values in Table 1, as follows: 


$$
\begin{aligned}
& \tilde{P}_{*}=\left\{\left\langle c_{1},([0.9627,0.9672],[0.0821,0.2512])\right\rangle,\right. \\
&\left\langle c_{2},([0.0411,0.0895],[0.9044,0.9901])\right\rangle, \\
&\left\langle c_{3},([0.0722,0.2072],[0.9024,0.9713])\right\rangle, \\
&\left\langle c_{4},([0.1041,0.3215],[0.9222,0.9365])\right\rangle, \\
&\left\langle c_{5},([0.8971,0.9274],[0.2174,0.4418])\right\rangle, \\
&\left\langle c_{6},([0.0991,0.1836],[0.9664,0.9709])\right\rangle, \\
&\left\langle c_{7},([0.0476,0.1758],[0.9844,0.9868])\right\rangle, \\
&\left.\left\langle c_{8},([0.0452,0.0995],[0.9949,0.9950])\right\rangle\right\}, \\
& \tilde{P}_{\#}=\left\{\left\langle c_{1},([0.4806,0.5752],[0.5695,0.7560])\right\rangle,\right.\left\langle c_{2},([0.6988,0.8868],[0.2599,0.2600])\right\rangle, \\
&\left\langle c_{3},([0.5592,0.6052],[0.4901,0.7692])\right\rangle, \\
&\left\langle c_{4},([0.5983,0.7577],[0.4559,0.6340])\right\rangle, \\
&\left\langle c_{5},([0.2174,0.4450],[0.4450,0.8899])\right\rangle, \\
&\left\langle c_{6},([0.3848,0.9866],[0.1625,0.1924])\right\rangle, \\
&\left\langle c_{7},([0.7934,0.9245],[0.2915,0.3642])\right\rangle, \\
&\left.\left\langle c_{8},([0.5675,0.7005],[0.2444,0.4784])\right\rangle\right\} .
\end{aligned}
$$

Furthermore, the intervals of the indeterminacy degrees corresponding to each $\tilde{p}_{* j}$ in $\tilde{P}_{*}$ were calculated as follows: $\left[\pi_{* 1}^{-}, \pi_{* 1}^{+}\right]=[0.0377,0.2578], \quad\left[\pi_{* 2}^{-}, \pi_{* 2}^{+}\right]=[0.1081,0.4247]$, $\left[\pi_{* 3}^{-}, \pi_{* 3}^{+}\right]=[0.1168,0.4248], \quad\left[\pi_{* 4}^{-}, \pi_{* 4}^{+}\right]=[0.1400,0.3724]$, $\left[\pi_{* 5}^{-}, \pi_{* 5}^{+}\right]=[0.0000,0.3846], \quad\left[\pi_{* 6}^{-}, \pi_{* 6}^{+}\right]=[0.1538,0.2372]$, $\left[\pi_{* 7}^{-}, \pi_{* 7}^{+}\right]=[0.0000,0.1694]$, and $\left[\pi_{* 8}^{-}, \pi_{* 8}^{+}\right]=[0.0086,0.0902]$. The intervals of the indeterminacy degrees corresponding to each $\tilde{p}_{\# j}$ in $\tilde{P}_{\#}$ were acquired as follows: $\left[\pi_{\# 1}^{-}, \pi_{\# 1}^{+}\right]=[0.3124$, $0.6669], \quad\left[\pi_{\# 2}^{-}, \pi_{\# 2}^{+}\right]=[0.3821,0.6664], \quad\left[\pi_{\# 3}^{-}, \pi_{\# 3}^{+}\right]=[0.2051$, $0.6687], \quad\left[\pi_{\# 4}^{-}, \pi_{\# 4}^{+}\right]=[0.1547,0.6589], \quad\left[\pi_{\# 5}^{-}, \pi_{\# 5}^{+}\right]=[0.1003$, $0.8687], \quad\left[\pi_{\# 6}^{-}, \pi_{\# 6}^{+}\right]=[0.0000,0.9086], \quad\left[\pi_{\# 7}^{-}, \pi_{\# 7}^{+}\right]=[0.1125$, $0.5344]$, and $\left[\pi_{\# 8}^{-}, \pi_{\# 8}^{+}\right]=[0.5296,0.7863]$.

In Step I.4, the information energies of all IVPF characteristics $\tilde{P}_{i}$ in $\tilde{\mathrm{p}}$ were computed using (19); the obtained results are as follows: $E\left(\tilde{P}_{1}\right)=0.6541, E\left(\tilde{P}_{2}\right)=0.6547, E\left(\tilde{P}_{3}\right)=$ 0.6856 , and $E\left(\tilde{P}_{4}\right)=0.6831$. Additionally, the information energies of $\tilde{P}_{*}$ and $\tilde{P}_{\#}$ for the displaced ideal IVPF solutions were acquired as follows: $E\left(\tilde{P}_{*}\right)=0.8556$ and $E\left(\tilde{P}_{\#}\right)=$ 0.5430 . Consider $E\left(\tilde{P}_{1}\right)$ as an example:

$$
\begin{aligned}
E\left(\tilde{P}_{1}\right)= & \frac{1}{2} \cdot\left[0 . 1 4 0 4 \cdot \left((0.4806)^{4}+(0.5752)^{4}+(0.5664)^{4}\right.\right. \\
& \left.+(0.7560)^{4}+(0.3123)^{4}+(0.6694)^{4}\right)+0.1252 \\
& \cdot\left((0.6988)^{4}+(0.8868)^{4}+(0.2599)^{4}+(0.2600)^{4}\right. \\
& \left.+(0.3822)^{4}+(0.6663)^{4}\right)+0.1090 \cdot\left((0.1146)^{4}\right. \\
& +(0.2072)^{4}+(0.9024)^{4}+(0.9713)^{4}+(0.1166)^{4} \\
& \left.+(0.4154)^{4}\right)+0.0839 \cdot\left((0.1041)^{4}+(0.7577)^{4}\right. \\
& \left.+(0.5653)^{4}+(0.6340)^{4}+(0.1546)^{4}+(0.8183)^{4}\right) \\
& +0.1361 \cdot\left((0.8971)^{4}+(0.9274)^{4}+(0.2174)^{4}\right. \\
& \left.+(0.4418)^{4}+(0.0000)^{4}+(0.3043)^{4}\right)+0.1252
\end{aligned}
$$

$$
\begin{aligned}
& \cdot\left((0.0991)^{4}+(0.1836)^{4}+(0.9064)^{4}+(0.9703)^{4}\right. \\
& \left.+(0.1574)^{4}+(0.4107)^{4}\right)+0.1408 \cdot\left((0.2198)^{4}\right. \\
& +(0.3513)^{4}+(0.5339)^{4}+(0.6189)^{4}+(0.7025)^{4} \\
& \left.+(0.8165)^{4}\right)+0.1394 \cdot\left((0.0452)^{4}+(0.0995)^{4}\right. \\
& \left.\left.+(0.9949)^{4}+(0.9950)^{4}+(0.0111)^{4}+(0.0904)^{4}\right)\right] \\
= & 0.6541 .
\end{aligned}
$$

In Steps I.5 and I.6, the correlations between $\tilde{P}_{i}$ and $\tilde{P}_{*}$ for all $z_{i} \in Z$ were determined using (20); the obtained results are as follows: $R\left(\tilde{P}_{1}, \tilde{P}_{*}\right)=0.5601, R\left(\tilde{P}_{2}, \tilde{P}_{*}\right)=0.5343, R\left(\tilde{P}_{3}, \tilde{P}_{*}\right)=$ 0.6685 , and $R\left(\tilde{P}_{4}, \tilde{P}_{*}\right)=0.5508$. Next, using (21), the correlation coefficients were computed as follows: $K\left(\tilde{P}_{1}, \tilde{P}_{*}\right)=$ $0.7487, K\left(\tilde{P}_{2}, \tilde{P}_{*}\right)=0.7138, K\left(\tilde{P}_{3}, \tilde{P}_{*}\right)=0.8729$, and $K\left(\tilde{P}_{4}\right.$, $\left.\tilde{P}_{*}\right)=0.7205$. Furthermore, the correlations between $\tilde{P}_{i}$ and $\tilde{P}_{\#}$ for each $z_{i}$ were acquired as follows: $R\left(\tilde{P}_{1}, \tilde{P}_{\#}\right)=0.3002$, $R\left(\tilde{P}_{2}, \tilde{P}_{\#}\right)=0.3353, \quad R\left(\tilde{P}_{3}, \tilde{P}_{\#}\right)=0.2950$, and $R\left(\tilde{P}_{4}, \tilde{P}_{\#}\right)=$ 0.3933 . The correlation coefficients for each $\tilde{P}_{i}$ were derived as follows: $K\left(\tilde{P}_{1}, \tilde{P}_{\#}\right)=0.5037, K\left(\tilde{P}_{2}, \tilde{P}_{\#}\right)=0.5624, K\left(\tilde{P}_{3}\right.$, $\left.\tilde{P}_{\#}\right)=0.4835$, and $K\left(\tilde{P}_{4}, \tilde{P}_{\#}\right)=0.6457$. Taking $R\left(\tilde{P}_{1}, \tilde{P}_{*}\right)$ and $K\left(\tilde{P}_{1}, \tilde{P}_{*}\right)$ for example:

$$
\begin{aligned}
R\left(\tilde{P}_{1}, \tilde{P}_{*}\right)= & \frac{1}{2} \cdot\left[0 . 1 4 0 4 \cdot \left((0.4806)^{2} \cdot(0.9627)^{2}+(0.5752)^{2}\right.\right. \\
& \cdot(0.9672)^{2}+(0.5664)^{2} \cdot(0.0821)^{2}+(0.7560)^{2} \\
& \cdot(0.2512)^{2}+(0.3123)^{2} \cdot(0.0377)^{2}+(0.6694)^{2} \\
& \left.\left.\cdot(0.2578)^{2}\right)\right]+0.1252 \cdot\left((0.6988)^{2} \cdot(0.0411)^{2}\right. \\
& +(0.8868)^{2} \cdot(0.0895)^{2}+(0.2599)^{2} \cdot(0.9044)^{2} \\
& +(0.2600)^{2} \cdot(0.9901)^{2}+(0.3822)^{2} \cdot(0.1081)^{2} \\
& \left.+(0.6663)^{2} \cdot(0.4247)^{2}\right)+0.1090 \cdot\left((0.1146)^{2}\right. \\
& \cdot(0.0722)^{2}+(0.2072)^{2} \cdot(0.2072)^{2}+(0.9024)^{2} \\
& \cdot(0.9024)^{2}+(0.9713)^{2} \cdot(0.9713)^{2}+(0.1166)^{2} \\
& \left.\cdot(0.1168)^{2}+(0.4154)^{2} \cdot(0.4248)^{2}\right)+0.0839 \\
& \cdot\left((0.1041)^{2} \cdot(0.1041)^{2}+(0.7577)^{2} \cdot(0.3215)^{2}\right. \\
& +(0.5653)^{2} \cdot(0.9222)^{2}+(0.6340)^{2} \cdot(0.9365)^{2} \\
& \left.+(0.1546)^{2} \cdot(0.1400)^{2}+(0.8183)^{2} \cdot(0.3724)^{2}\right) \\
& +0.1361 \cdot\left((0.8971)^{2} \cdot(0.8971)^{2}+(0.9274)^{2}\right. \\
& \cdot(0.9274)^{2}+(0.2174)^{2} \cdot(0.2174)^{2}+(0.4418)^{2} \\
& \cdot(0.4418)^{2}+(0.0000)^{2} \cdot(0.0000)^{2}+(0.3043)^{2} \\
& \left.\cdot(0.3846)^{2}\right)+0.1252\left((0.0991)^{2} \cdot(0.0991)^{2}\right. \\
& +(0.1836)^{2} \cdot(0.1836)^{2}+(0.9064)^{2} \cdot(0.9664)^{2} \\
& +(0.9703)^{2} \cdot(0.9709)^{2}+(0.1574)^{2} \cdot(0.1538)^{2} \\
& \left.+(0.4107)^{2} \cdot(0.2372)^{2}\right)+0.1408 \cdot\left((0.2198)^{2}\right. \\
& \cdot(0.0476)^{2}+(0.3513)^{2} \cdot(0.1758)^{2}+(0.5339)^{2} \\
& \cdot(0.9844)^{2}+(0.6189)^{2} \cdot(0.9868)^{2}+(0.7025)^{2}
\end{aligned}
$$




$$
\begin{aligned}
& \left.\cdot(0.0000)^{2}+(0.8165)^{2} \cdot(0.1694)^{2}\right)+0.1394 \\
& \cdot\left((0.0452)^{2} \cdot(0.0452)^{2}+(0.0995)^{2} \cdot(0.0995)^{2}\right. \\
& +(0.9949)^{2} \cdot(0.9949)^{2}+(0.9950)^{2} \cdot(0.9950)^{2} \\
& \left.+(0.0111)^{2} \cdot(0.0086)^{2}+(0.0904)^{2} \cdot(0.0902)^{2}\right) \\
= & 0.5601,
\end{aligned}
$$$$
K\left(\tilde{P}_{1}, \tilde{P}_{*}\right)=\frac{0.5601}{\sqrt{0.6541 \cdot 0.8556}}=0.7487 .
$$

In Step I.7, using (24), the correlation-based closeness indices for all $z_{i} \in Z$ were determined as follows: $I_{d}\left(\tilde{P}_{1}\right)=$ $0.7487 /(0.7487+0.5037)=0.5978, I_{d}\left(\tilde{P}_{2}\right)=0.5593, I_{d}\left(\tilde{P}_{3}\right)=$
0.6435 , and $I_{d}\left(\tilde{P}_{4}\right)=0.5274$. In Step I.8, the ultimate priority ranking $z_{3}>z_{1}>z_{2}>z_{4}$ was acquired by sorting each $I_{d}\left(\tilde{P}_{i}\right)$ value in descending order. Therefore, alternative $z_{3}$, i.e., the balanced cantilever method, should be selected as the most appropriate bridge construction method among the four candidate alternatives for the Suhua Highway Alternative Road Project.

Next, the developed methodology with Algorithm 2 was applied to solve the same selection problem of bridge construction methods. Note that Steps II.1 and II.2 are the same as Steps I.1 and I.2 of Algorithm 1. In Step II.3, the correlation-based closeness index $I_{f}\left(\tilde{P}_{i}\right)$ of each IVPF characteristic $\tilde{P}_{i}$ for all $z_{i} \in Z$ can be determined using (26), and the obtained results are as follows: $I_{f}\left(\tilde{P}_{1}\right)=0.7424, I_{f}\left(\tilde{P}_{2}\right)=0.7292, I_{f}\left(\tilde{P}_{3}\right)=$ 0.8269 , and $I_{f}\left(\tilde{P}_{4}\right)=0.6673$. Using $I_{f}\left(\tilde{P}_{1}\right)$ as an example,

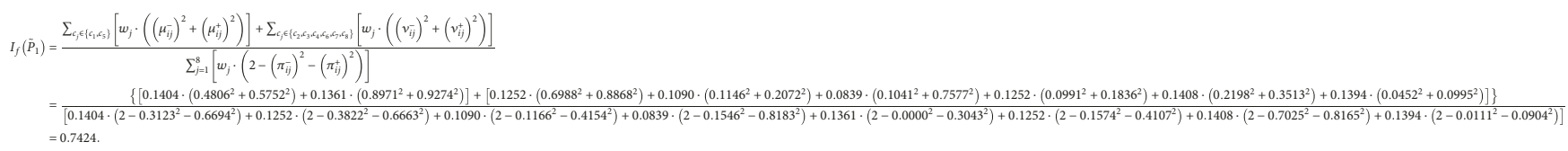

In Step II.4, the ultimate priority ranking $z_{3}>z_{1}>z_{2}>z_{4}$ was obtained by sorting each $I_{f}\left(\tilde{P}_{i}\right)$ value in descending order. This ranking result is in accordance with that yielded by Algorithm 1. Therefore, the best choice for the bridge construction case is the balanced cantilever method $\left(z_{3}\right)$.

4.3. Comparative Discussions. This subsection conducts a comparative analysis of the obtained results yielded by the developed methodology with Algorithms 1 and 2. This analysis focuses on the respective solution results based on anchored judgments with displaced ideals and fixed ideals.

It was not necessary for decision-makers to have determined the correlation coefficients $K\left(\tilde{P}_{i}, \tilde{P}_{+}\right)$and $K\left(\tilde{P}_{i}, \tilde{P}_{-}\right)$ when applying Algorithm 2 to address MCDA problems. Nevertheless, the current comparative study additionally calculated $K\left(\tilde{P}_{i}, \tilde{P}_{+}\right)$and $K\left(\tilde{P}_{i}, \tilde{P}_{-}\right)$to facilitate detailed comparisons of Algorithms 1 and 2 on a consistent basis. To demonstrate the differences among the relevant outcomes generated by the proposed methodology from the perspectives of displaced and fixed ideals, this paper highlights the summarized results of pairwise comparisons with respect to correlation coefficients and correlation-based closeness indices in the following three figures.

Figure 1 depicts the comparison results of the correlation coefficients $K\left(\tilde{P}_{i}, \tilde{P}_{*}\right)$ and $K\left(\tilde{P}_{i}, \tilde{P}_{+}\right)$with respect to the positive-ideal IVPF solutions $z_{*}$ and $z_{+}$. As revealed in this figure, there are no evident differences between $K\left(\tilde{P}_{i}, \tilde{P}_{*}\right)$ and $K\left(\tilde{P}_{i}, \tilde{P}_{+}\right)$in relation to each alternative. Thus, the correlations between the IVPF characteristics $\tilde{P}_{i}$ and $\tilde{P}_{*}$ are very similar to the correlations between $\tilde{P}_{i}$ and $\tilde{P}_{+}$in the bridge construction case. Moreover, the values of $K\left(\tilde{P}_{i}, \tilde{P}_{*}\right)$ and $K\left(\tilde{P}_{i}, \tilde{P}_{+}\right)$show very similar distributions and trends with respect to the four alternatives. More specifically, the two similar rankings $z_{3}>z_{1}>z_{4}>z_{2}$ (in accordance with $K\left(\tilde{P}_{3}\right.$, $\left.\left.\tilde{P}_{*}\right)>K\left(\tilde{P}_{1}, \tilde{P}_{*}\right)>K\left(\tilde{P}_{4}, \tilde{P}_{*}\right)>K\left(\tilde{P}_{2}, \tilde{P}_{*}\right)\right)$ and $z_{3}>z_{1}>z_{2}>$ $z_{4}$ (in the light of $K\left(\tilde{P}_{3}, \tilde{P}_{+}\right)>K\left(\tilde{P}_{1}, \tilde{P}_{+}\right)>K\left(\tilde{P}_{2}, \tilde{P}_{+}\right)>K\left(\tilde{P}_{4}\right.$, $\left.\left.\tilde{P}_{+}\right)\right)$can be acquired by sorting the values of $K\left(\tilde{P}_{i}, \tilde{P}_{*}\right)$ and $K\left(\tilde{P}_{i}, \tilde{P}_{+}\right)$separately in descending order. On average, the extent of each alternative $z_{i}$ associated with the displaced positive-ideal IVPF solution $z_{*}$ is very close to that of the $z_{i}$ associated with the fixed positive-ideal IVPF solution $z_{+}$, based on the comparison results via correlation coefficients of IVPF characteristics.

Figure 2 reveals the summarized comparisons of the correlation coefficients $K\left(\tilde{P}_{i}, \tilde{P}_{\#}\right)$ and $K\left(\tilde{P}_{i}, \tilde{P}_{-}\right)$in terms of the negative-ideal IVPF solutions $z_{\#}$ and $z_{-}$. The contrast between $K\left(\tilde{P}_{i}, \tilde{P}_{\#}\right)$ and $K\left(\tilde{P}_{i}, \tilde{P}_{-}\right)$is significantly different from the contrast between $K\left(\tilde{P}_{i}, \tilde{P}_{*}\right)$ and $K\left(\tilde{P}_{i}, \tilde{P}_{+}\right)$in Figure 1. Overall, the dissimilarity between the IVPF characteristics $\tilde{P}_{i}$ and $\tilde{P}_{\#}$ is relatively lower than that between $\tilde{P}_{i}$ and $\tilde{P}_{-}$in the bridge construction case. As can be expected, the $K\left(\tilde{P}_{i}, \tilde{P}_{\#}\right)$ values are markedly greater than the $K\left(\tilde{P}_{i}, \tilde{P}_{+}\right)$ values. The difference between $K\left(\tilde{P}_{i}, \tilde{P}_{\#}\right)$ and $K\left(\tilde{P}_{i}, \tilde{P}_{-}\right)$is obvious in general. However, they demonstrate a common distribution among the four alternatives. More precisely, by sorting the $K\left(\tilde{P}_{i}, \tilde{P}_{\#}\right)$ and $K\left(\tilde{P}_{i}, \tilde{P}_{-}\right)$values separately in ascending order, the same ranking $z_{3}>z_{1}>z_{2}>z_{4}$ can be obtained because $K\left(\tilde{P}_{3}, \tilde{P}_{\#}\right)<K\left(\tilde{P}_{1}, \tilde{P}_{\#}\right)<K\left(\tilde{P}_{2}, \tilde{P}_{\#}\right)<K$ $\left(\tilde{P}_{4}, \tilde{P}_{\#}\right)$ and $K\left(\tilde{P}_{3}, \tilde{P}_{-}\right)<K\left(\tilde{P}_{1}, \tilde{P}_{-}\right)<K\left(\tilde{P}_{2}, \tilde{P}_{-}\right)<K\left(\tilde{P}_{4}, \tilde{P}_{-}\right)$. 


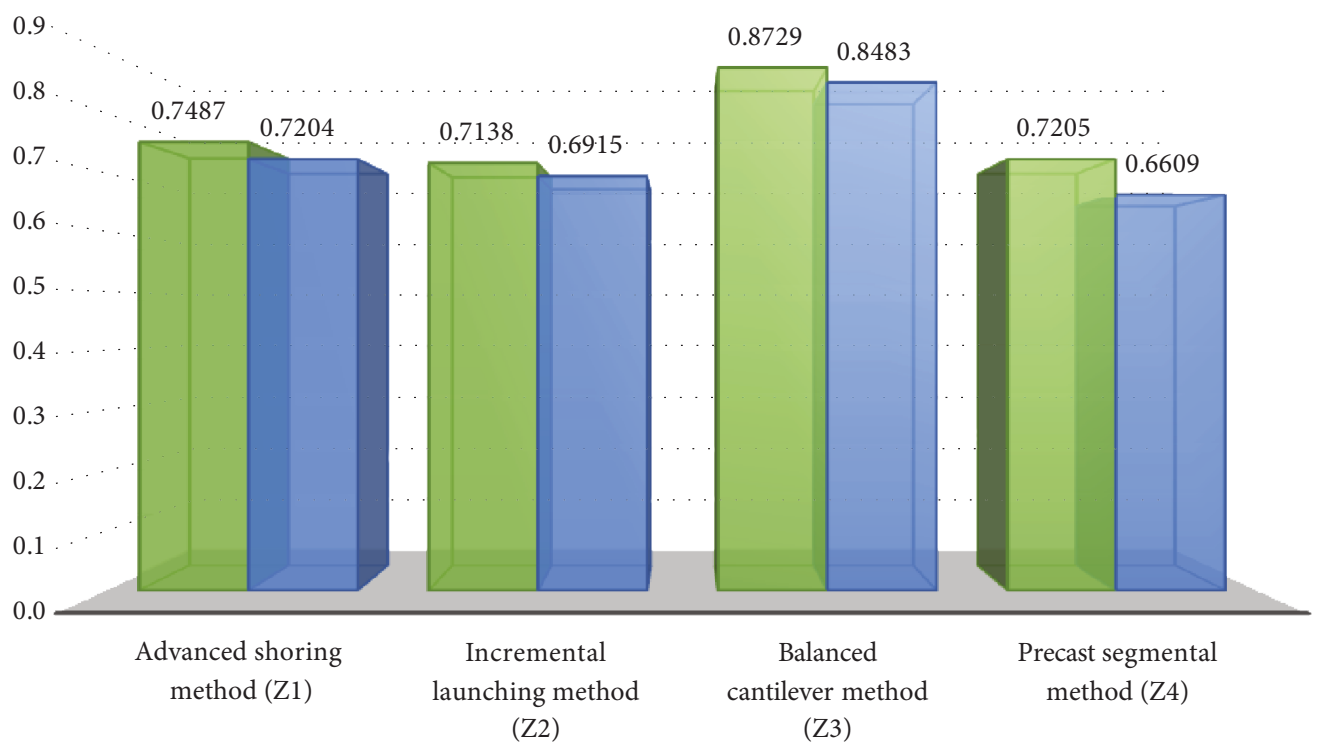

$\square$ Algorithm 1
$\square$ Algorithm 2

Figure 1: Comparison results for $K\left(\tilde{P}_{i}, \tilde{P}_{*}\right)$ and $K\left(\tilde{P}_{i}, \tilde{P}_{+}\right)$.

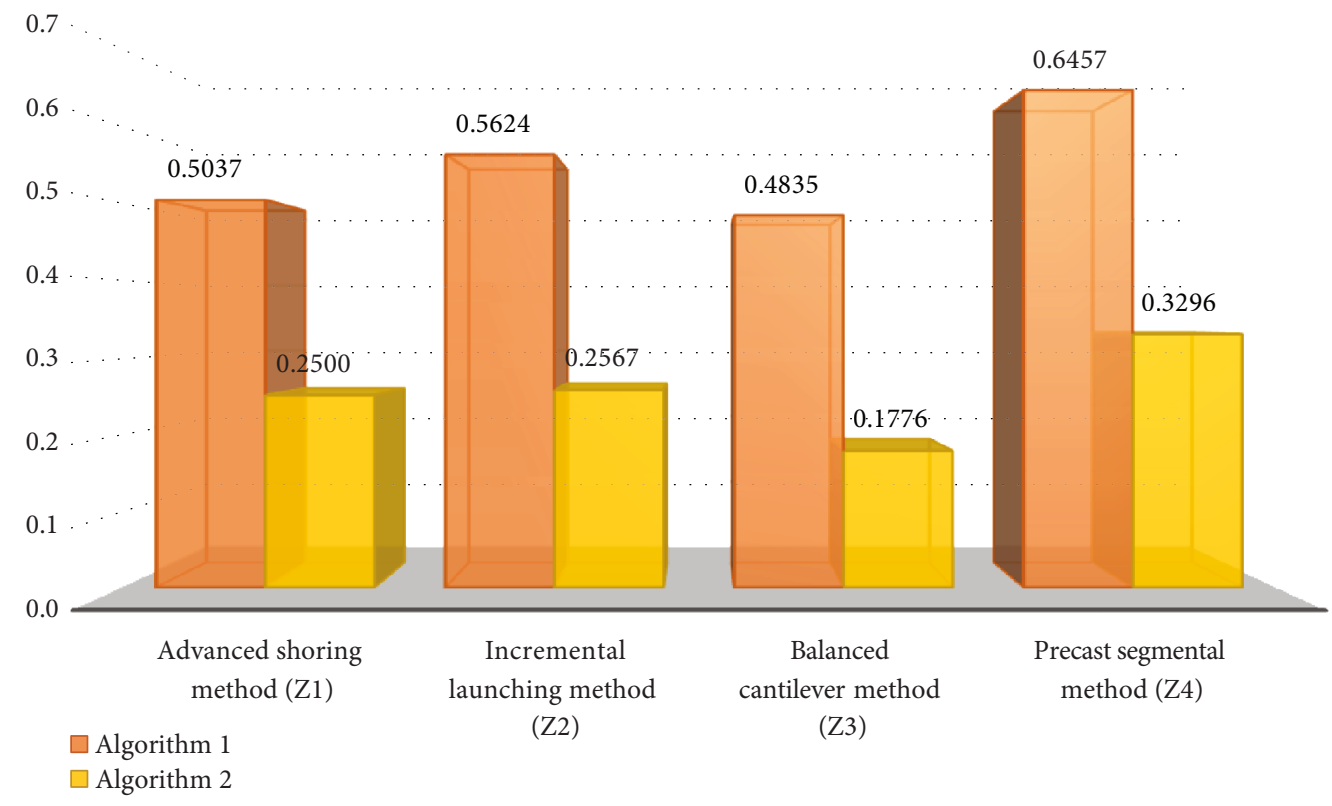

Figure 2: Comparison results for $K\left(\tilde{P}_{i}, \tilde{P}_{\#}\right)$ and $K\left(\tilde{P}_{i}, \tilde{P}_{-}\right)$.

Conversely, there is an evident distinction between $\tilde{P}_{\#}$ and $\tilde{P}_{-}$; however, the difference between $\tilde{P}_{*}$ and $\tilde{P}_{+}$is not significant everywhere. Thus, the difference between $K\left(\tilde{P}_{i}, \tilde{P}_{\#}\right)$ and $K\left(\tilde{P}_{i}, \tilde{P}_{-}\right)$should be obviously larger than that between $K\left(\tilde{P}_{i}, \tilde{P}_{*}\right)$ and $K\left(\tilde{P}_{i}, \tilde{P}_{+}\right)$. As seen in Figure 2 , the $K\left(\tilde{P}_{i}, \tilde{P}_{\#}\right)$ values are larger than the $K\left(\tilde{P}_{i}, \tilde{P}_{-}\right)$values with respect to the four alternatives. In contrast, the $K\left(\tilde{P}_{i}, \tilde{P}_{*}\right)$ values are close to the $K\left(\tilde{P}_{i}, \tilde{P}_{+}\right)$values for all alternatives, as indicated in Figure 1 .
Figure 3 shows the contrast of the correlation-based closeness indices $I_{d}\left(\tilde{P}_{i}\right)$ and $I_{f}\left(\tilde{P}_{i}\right)$. In the bridge construction case, the correlation-based closeness indices lie in the range from 0.52 to 0.65 based on anchored judgments with displaced ideals (i.e., using Algorithm 1) and from 0.66 to 0.83 based on anchored judgments with fixed ideals (i.e., using Algorithm 2). These indices have different ranges. However, they show a common distribution among the four alternatives. Specifically, by sorting the $I_{d}\left(\tilde{P}_{i}\right)$ and $I_{f}\left(\tilde{P}_{i}\right)$ values separately in descending order, the same ultimate 


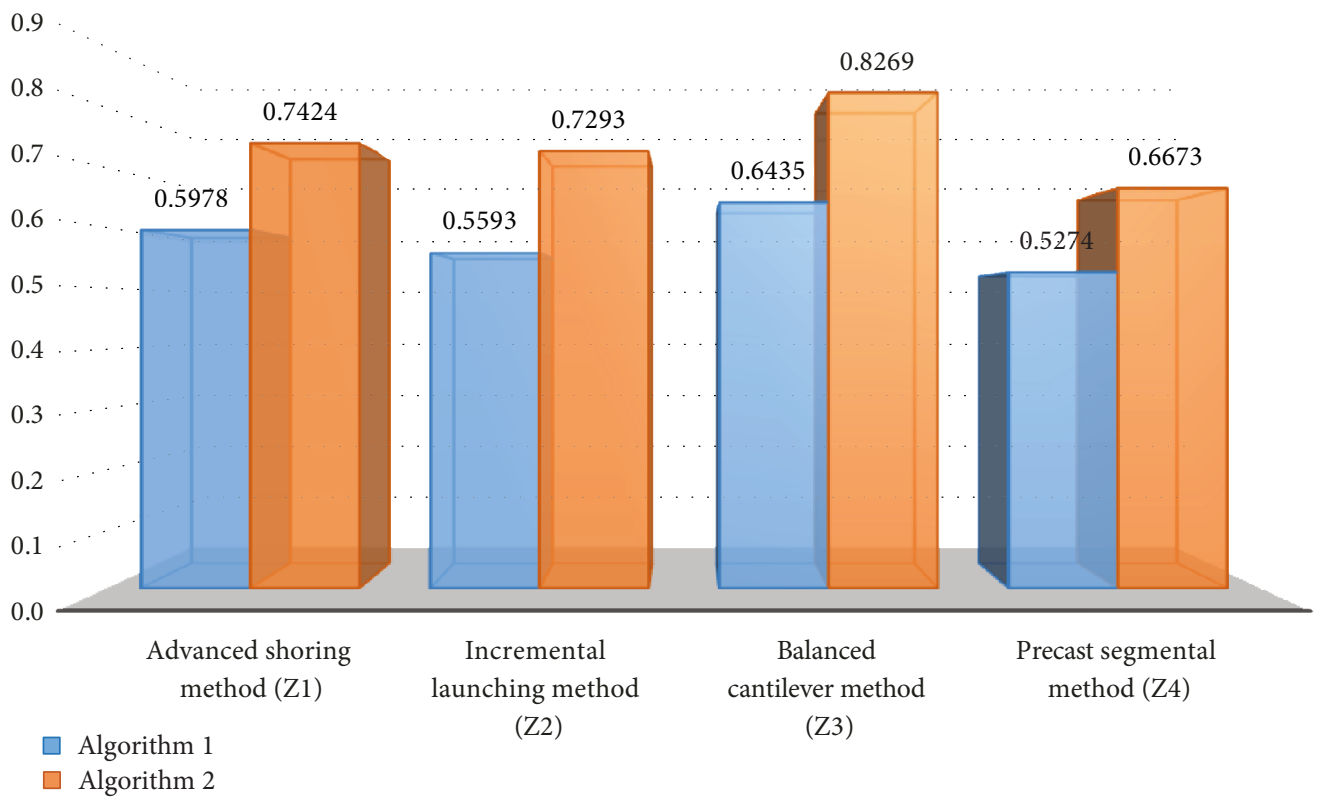

Figure 3: Comparison results for $I_{d}\left(\tilde{P}_{i}\right)$ and $I_{f}\left(\tilde{P}_{i}\right)$.

priority ranking $z_{3}>z_{1}>z_{2}>z_{4}$ can be obtained according to $I_{d}\left(\tilde{P}_{3}\right)>I_{d}\left(\tilde{P}_{1}\right)>I_{d}\left(\tilde{P}_{2}\right)>I_{d}\left(\tilde{P}_{4}\right)$ using Algorithm 1 and $I_{f}\left(\tilde{P}_{3}\right)>I_{f}\left(\tilde{P}_{1}\right)>I_{f}\left(\tilde{P}_{2}\right)>I_{f}\left(\tilde{P}_{4}\right)$ using Algorithm 2 . The feasibility and applicability of the proposed methodology with Algorithms 1 and 2 can be validated and supported through the practical application concerning the selection problem of bridge construction methods.

Based on the comparison results, the developed Algorithms 1 and 2 can generate a stable and steady ultimate priority ranking among competing alternatives. In particular, the implementation procedure of Algorithm 2 is significantly simpler and more effective than that of Algorithm 1. In contrast, with Algorithm 1 or other MCDA methods in the IVPF context, the developed Algorithm 2 can fully account for the information associated with IVPF evaluative ratings and importance weights in a convenient manner. However, Algorithm 2 can also produce an intuitively appealing and persuading solution result. In this respect, adopting Algorithm 2 as the main structure of the IVPF compromise approach is suggested, i.e., the usage of correlation-based closeness indices with respect to the fixed ideal IVPF solutions when addressing MCDA problems within the IVPF environment.

\section{Comparative Analysis}

This section attempts to conduct some comparisons of the proposed IVPF compromise approach with well-known and widely used compromise models as well as with different fuzzy MCDA methods in uncertain environments based on other nonstandard fuzzy sets to illustrate the advantages and effectiveness of the developed methodology. Furthermore, this section extends the applicability of the proposed methods to other application fields for enriching the practical potentials in the real world.
5.1. Comparison to Well-Known TOPSIS Methods. Consider that the TOPSIS methodology is the most widely used compromise model in the MCDA field. In particular, TOPSIS utilizes the distances to both the ideal and the negative-ideal solutions simultaneously to determine the closeness coefficient, which inspires this paper to develop the useful concept of correlation-based closeness indices. More importantly, both TOPSIS and the proposed approach belong to the compromise model. For these reasons, this subsection extends the classic TOPSIS to the IVPF environment to conduct a comprehensive comparative study.

In this subsection, the comparative analysis focuses on a comparison of the results yielded by the extended TOPSIS method and those obtained by the developed methodology. TOPSIS has been the most widely used compromise model in the last few decades. Because the proposed methods belong to the compromise model in nature, this paper attempts to extend the classic TOPSIS to the IVPF environment to address highly uncertain information based on IVPF sets to facilitate a comparative study.

Consider an MCDA problem involving the IVPF decision matrix $\tilde{p}=\left[\tilde{p}_{i j}\right]_{m \times n}$ and the weight vector $\mathrm{w}^{T}=\left(w_{1}, w_{2}\right.$, $\left.\cdots, w_{n}\right)^{T}$. The IVPF TOPSIS method is presented as a series of successive steps. First, let an IVPF value $\tilde{\rho}_{i j}$ denote the weighted evaluative rating of an alternative $z_{i} \in Z$; $\tilde{\rho}_{i j}$ is calculated as follows:

$$
\begin{gathered}
\tilde{\rho}_{i j}=w_{j} \cdot \tilde{p}_{i j}=\left(\left[\sqrt{1-\left(1-\left(\mu_{i j}^{-}\right)^{2}\right)^{w_{j}}},\right.\right. \\
\left.\left.\sqrt{1-\left(1-\left(\mu_{i j}^{+}\right)^{2}\right)^{w_{j}}}\right],\left[\left(v_{i j}^{-}\right)^{w_{j}},\left(v_{i j}^{+}\right)^{w_{j}}\right]\right) .
\end{gathered}
$$


Concerning anchored judgments with displaced ideals, let two IVPF values $\tilde{\rho}_{* j}$ and $\tilde{\rho}_{\# j}$ denote the weighted evaluative ratings of the displaced positive-ideal IVPF solution $z_{*}$ and the displaced negative-ideal IVPF solution $z_{\#}$, respectively. Using Definition $3, \tilde{\rho}_{* j}$ and $\tilde{\rho}_{\# j}$ are identified as follows:

$$
\begin{gathered}
\tilde{\rho}_{* j}= \begin{cases}\bigvee_{i=1}^{m} \tilde{\rho}_{i j} & \text { if } c_{j} \in C_{\mathrm{I}}, \\
\bigwedge_{i=1}^{m} \tilde{\rho}_{i j} & \text { if } c_{j} \in C_{\mathrm{II}},\end{cases} \\
\tilde{\rho}_{\# j}= \begin{cases}\bigwedge_{i=1}^{m} \tilde{\rho}_{i j} & \text { if } c_{j} \in C_{\mathrm{I}}, \\
\bigvee_{i=1}^{m} \tilde{\rho}_{i j} & \text { if } c_{j} \in C_{\mathrm{II}},\end{cases}
\end{gathered}
$$

where

$$
\begin{aligned}
\bigvee_{i=1}^{m} \tilde{\rho}_{i j}= & \left(\left[\sqrt{1-\left(1-\left(\max _{i=1}^{m} \mu_{i j}^{-}\right)^{2}\right)^{w_{j}}},\right.\right. \\
& \left.\sqrt{1-\left(1-\left(\max _{i=1}^{m} \mu_{i j}^{+}\right)^{2}\right)^{w_{j}}}\right], \\
& {\left.\left[\left(\min _{i=1}^{m} v_{i j}^{-}\right)^{w_{j}},\left(\min _{i=1}^{m} v_{i j}^{+}\right)^{w_{j}}\right]\right), } \\
\bigwedge_{i=1}^{m} \tilde{\rho}_{i j}= & \left(\left[\sqrt{1-\left(1-\left(\min _{i=1}^{m} \mu_{i j}^{-}\right)^{2}\right)^{w_{j}}},\right.\right. \\
& \left.\sqrt{1-\left(1-\left(\min _{i=1}^{m} \mu_{i j}^{+}\right)^{2}\right)^{w_{j}}}\right], \\
& {\left[\left(\max _{i=1}^{m} v_{i j}^{-}\right)^{w_{j}},\left(\max _{i=1}^{m} v_{i j}^{+}\right)^{w_{j}}\right] }
\end{aligned}
$$

Next, the distances $D\left(\tilde{\rho}_{i j}, \tilde{\rho}_{* j}\right)$ and $D\left(\tilde{\rho}_{i j}, \tilde{\rho}_{\# j}\right)$ between $\tilde{\rho}_{i j}$ and $\tilde{\rho}_{* j}$ and between $\tilde{\rho}_{i j}$ and $\tilde{\rho}_{\# j}$, respectively, can be computed using Definition 4 . Based on the obtained $D\left(\tilde{\rho}_{i j}, \tilde{\rho}_{* j}\right)$, the separation $D_{i}^{*}$ of each alternative $z_{i} \in Z$ from the displaced positive-ideal IVPF solution $z_{*}$ is given as follows:

$$
D_{i}^{*}=\frac{1}{n} \sum_{j=1}^{n} D\left(\tilde{\rho}_{i j}, \tilde{\rho}_{* j}\right) .
$$

Similarly, according to the obtained $D\left(\tilde{\rho}_{i j}, \tilde{\rho}_{\# j}\right)$, the separation $D_{i}^{\#}$ of each alternative $z_{i} \in Z$ from the displaced negative-ideal IVPF solution $z_{\#}$ is given as follows:

$$
D_{i}^{\#}=\frac{1}{n} \sum_{j=1}^{n} D\left(\tilde{\rho}_{i j}, \tilde{\rho}_{\# j}\right) .
$$

Let $C C_{i}^{d}$ denote the closeness coefficient of an alternative $z_{i}$ with respect to the displaced ideal IVPF solutions; it is defined as follows:

$$
C C_{i}^{d}=\frac{D_{i}^{\#}}{D_{i}^{*}+D_{i}^{\#}}
$$

According to $C C_{i}^{d}$ in descending order, the ultimate priority orders among $m$ candidate alternatives can be determined.

Concerning anchored judgments with fixed ideals, let two IVPF values $\tilde{\rho}_{+j}$ and $\tilde{\rho}_{-j}$ denote the weighted evaluative ratings of the fixed positive-ideal IVPF solution $z_{+}$and the fixed negative-ideal IVPF solution $z_{-}$, respectively; they are given as follows:

$$
\begin{aligned}
& \tilde{\rho}_{+j}=w_{j} \cdot \tilde{p}_{+j}= \begin{cases}([1,1],[0,0]) & \text { if } c_{j} \in C_{\mathrm{I}}, \\
([0,0],[1,1]) & \text { if } c_{j} \in C_{\mathrm{II}},\end{cases} \\
& \tilde{\rho}_{-j}=w_{j} \cdot \tilde{p}_{-j}= \begin{cases}([0,0],[1,1]) & \text { if } c_{j} \in C_{\mathrm{I}}, \\
([1,1],[0,0]) & \text { if } c_{j} \in C_{\mathrm{II}} .\end{cases}
\end{aligned}
$$

After determining the distances $D\left(\tilde{\rho}_{i j}, \tilde{\rho}_{+j}\right)$ and $D\left(\tilde{\rho}_{i j}\right.$, $\left.\tilde{\rho}_{-j}\right)$ for each $\tilde{\rho}_{i j}$, the separations $D_{i}^{+}$and $D_{i}^{-}$of each alternative $z_{i}$ from the fixed ideal IVPF solutions $z_{+}$and $z_{-}$, respectively, can be obtained as follows:

$$
\begin{aligned}
& D_{i}^{+}=\frac{1}{n} \sum_{j=1}^{n} D\left(\tilde{\rho}_{i j}, \tilde{\rho}_{+j}\right), \\
& D_{i}^{-}=\frac{1}{n} \sum_{j=1}^{n} D\left(\tilde{\rho}_{i j}, \tilde{\rho}_{-j}\right) .
\end{aligned}
$$

The closeness coefficient $C C_{i}^{f}$ of an alternative $z_{i}$ with respect to the fixed ideal IVPF solutions is calculated as follows:

$$
C C_{i}^{f}=\frac{D_{i}^{-}}{D_{i}^{+}+D_{i}^{-}}
$$

The ultimate priority orders among $m$ candidate alternatives can be acquired in accordance with the $C C_{i}^{f}$ values in descending order.

Employing the IVPF TOPSIS method to address the selection problem of bridge construction methods, the obtained results of the separations $\left(D_{i}^{*}, D_{i}^{\#}, D_{i}^{+}\right.$, and $\left.D_{i}^{-}\right)$ and the closeness coefficients $\left(C C_{i}^{d}\right.$ and $\left.C C_{i}^{f}\right)$ are revealed in Table 2. According to the $C C_{i}^{d}$ values, the ultimate priority ranking $z_{3}>z_{2}>z_{1}>z_{4}$ was acquired based on anchored judgments with displaced ideals. Moreover, the ranking result $z_{3}>z_{1}>z_{2}>z_{4}$ was determined by sorting each $C C_{i}^{f}$ value based on anchored judgments with fixed ideals. The best alternative for the bridge construction case is the balanced cantilever method $\left(z_{3}\right)$, which is consistent with the result yielded by the proposed methodology.

5.2. Comparison to Relevant Nonstandard Fuzzy Models. This subsection includes a comparative discussion with other nonstandard fuzzy models to demonstrate the advantages 
TABLE 2: Separations and closeness coefficients yielded by the IVPF TOPSIS method.

\begin{tabular}{cccccccccc}
\hline & \multicolumn{3}{c}{ Anchored judgments with } & \multicolumn{4}{c}{ Anchored judgments with } \\
$z_{i}$ & \multicolumn{3}{c}{ displaced ideals } & \multicolumn{4}{c}{ fixed ideals } \\
& $D_{i}^{*}$ & $D_{i}^{\#}$ & $C C_{i}^{d}$ & Rank & $D_{i}^{+}$ & $D_{i}^{-}$ & $C C_{i}^{f}$ & Rank \\
\hline$z_{1}$ & 0.1002 & 0.1335 & 0.5711 & 3rd & 0.2863 & 0.7759 & 0.7304 & 2nd \\
$z_{2}$ & 0.1024 & 0.1425 & 0.5818 & 2nd & 0.2925 & 0.7883 & 0.7294 & 3 rd \\
$z_{3}$ & 0.0545 & 0.1816 & 0.7691 & 1 st & 0.2358 & 0.8080 & 0.7741 & 1 st \\
$z_{4}$ & 0.1221 & 0.1151 & 0.4854 & 4 th & 0.3049 & 0.7499 & 0.7109 & 4 th \\
\hline
\end{tabular}

of the proposed IVPF compromise approaches using correlation-based closeness indices.

The comparative analysis focuses on investigations of the obtained results yielded by different MCDA methods based on other nonstandard fuzzy sets. As mentioned previously, Chen $[18,59,60]$ and Wang and Chen [52] explored the selection problem of bridge construction methods for the Suhua Highway Alternative Road Project. The decision environments discussed in the above research were based on IVIF sets. The concept of IVPF sets can be considered an extension of IVIF sets [40, 43]. From this perspective, this paper further draws a comparison with these nonstandard fuzzy models, consisting of the nonlinear assignment-based method [59], the IVIF qualitative flexible multiple-criteria method (QUALIFLEX) [60], the extended linear assignment method [18], and the likelihood-based assignment method [52]. Additionally, Chen [42] developed an IVPF outranking method with a closeness-based assignment model and examined its feasibility and effectiveness using the selection problem of bridge construction methods. Thus, this paper also compares the obtained result yielded by the IVPF outranking method. With respect to the bridge construction case, Table 3 indicates the summary application results using the aforementioned comparative approaches and the proposed methodology. In particular, the data types of evaluative ratings and criterion weights, the obtained ranking results, and the category of MCDA models among the comparative methods are contrasted in this table.

According to the side-by-side comparisons presented in Table 3, the nonlinear assignment-based method, the IVIF QUALIFLEX method, the extended linear assignment method, the likelihood-based assignment method (with Algorithms A and B), and the IVPF outranking method belong to the outranking model. However, the IVPF TOPSIS method with respect to displaced/fixed ideals and the proposed IVPF compromise approach (with Algorithms 1 and 2) belong to the compromise model. As revealed in this table, the assignment-based methods introduced by Chen $[18,42,59]$ and Wang and Chen [52] produced two similar ultimate ranking results: $z_{1}>z_{3}>z_{2}>z_{4}$ and $z_{1}>z_{3}>z_{4}>$ $z_{2}$. Although these results are somewhat different from the rankings obtained using the compromise-based methods (i.e., the IVPF TOPSIS method and the proposed methodology), the relatively better alternatives (i.e., $z_{1}$ and $z_{3}$ ) and the relatively worse alternatives (i.e., $z_{2}$ and $z_{4}$ ) yielded by the assignment- and compromise-based methods are the same.
Conversely, significantly different results $z_{4}>z_{3}>z_{1}>z_{2}$ and $z_{2}>z_{3}>z_{1}>z_{4}$ were acquired using the IVIF QUALIFLEX method. These inconsistent rankings can result from the distinct data types of evaluative ratings and/or the different preference structures of criterion importance. For example, the nonlinear assignment-based method, the IVIF QUALIFLEX method, and the likelihood-based assignment method with Algorithm B are aimed at coping with IVIF MCDA problems involving incomplete preference information. More precisely, the importance weights of criteria are unknown and might even conflict a priori. In contrast, the extended linear assignment method and the likelihoodbased assignment method with Algorithm A utilized IVIF values to express criterion weights. Furthermore, the importance weights were represented using IVPF values in the IVPF outranking method. The IVPF TOPSIS method and the proposed methodology evaluated criterion weights as ordinary (i.e., crisp and nonfuzzy) values. Therefore, there are evident discriminations among the preference structures of criterion importance adopted in these comparative methods. Additionally, the assignment-based methods established the evaluative ratings based on IVIF sets, whereas the compromise-based methods utilized IVPF sets to construct the evaluative ratings. The above discussions can be a source of the ranking differentiation in the comparison results.

Concerning the ultimate priority ranking results, decision-makers often consider the relatively better alternatives further to make a final decision. For the most part, the alternatives that are top ranked and second ranked in the ultimate priority ranking deserve serious consideration. As indicated in Table $3, z_{1}$ and $z_{3}$ are relatively better alternatives in relation to most of the comparative methods. The assignment-based methods produced the ranking result $z_{1}$ $>z_{3}$, but the compromise-based methods generated an opposite result $z_{3}>z_{1}$. To investigate the validity and justifiability of these conflicting results, the concept of extended score functions within the IVPF environment was employed to facilitate judgments about the priority orders of $z_{1}$ and $z_{3}$.

By modifying Yu et al.'s score function (2012) based on IVIF sets for adapting to the IVPF environment, the extended score function $S\left(\tilde{\rho}_{i j}\right)$ of an IVPF-weighted evaluative rating $\tilde{\rho}_{i j}$ is computed as follows:

$$
\begin{aligned}
S\left(\tilde{\rho}_{i j}\right)= & \frac{1}{4}\left(2+\left(\sqrt{1-\left(1-\left(\mu_{i j}^{-}\right)^{2}\right)^{w_{j}}}\right)^{2}\right. \\
& +\left(\sqrt{1-\left(1-\left(\mu_{i j}^{+}\right)^{2}\right)^{w_{j}}}\right)^{2}-\left(\left(v_{i j}^{-}\right)^{w_{j}}\right)^{2} \\
& \left.-\left(\left(v_{i j}^{+}\right)^{w_{j}}\right)^{2}\right)=\frac{1}{4}\left(4-\left(1-\left(\mu_{i j}^{-}\right)^{2}\right)^{w_{j}}\right. \\
& \left.-\left(1-\left(\mu_{i j}^{+}\right)^{2}\right)^{w_{j}}-\left(v_{i j}^{-}\right)^{2 w_{j}}-\left(v_{i j}^{+}\right)^{2 w_{j}}\right),
\end{aligned}
$$

where $0 \leq S\left(\tilde{\rho}_{i j}\right) \leq 1$. A comparable value of $\tilde{\rho}_{i j}$ can be acquired using the extended score function $S\left(\tilde{\rho}_{i j}\right)$. Concerning a benefit 
TABLE 3: Comparative analysis of the obtained results in the bridge construction case.

\begin{tabular}{|c|c|c|c|c|c|}
\hline Source & Methods & $\begin{array}{l}\text { Evaluative } \\
\text { rating }\end{array}$ & Criterion weight & Ranking result & Model category \\
\hline Chen [59] & Nonlinear assignment-based method & IVIF values & Incomplete information & $z_{1}>z_{3}>z_{2}>z_{4}$ & Outranking model \\
\hline \multirow[b]{2}{*}{ Chen $[60]$} & IVIF QUALIFLEX method & IVIF values & Incomplete information & $z_{4}>z_{3}>z_{1}>z_{2}$ & Outranking model \\
\hline & & IVIF values & $\begin{array}{l}\text { Incomplete and inconsistent } \\
\text { information }\end{array}$ & $z_{2}>z_{3}>z_{1}>z_{4}$ & Outranking model \\
\hline Chen [18] & Extended linear assignment method & IVIF values & IVIF values & $z_{1}>z_{3}>z_{4}>z_{2}$ & Outranking model \\
\hline \multirow{2}{*}{$\begin{array}{l}\text { Wang and } \\
\text { Chen [52] }\end{array}$} & $\begin{array}{l}\text { Likelihood-based assignment method } \\
\text { with Algorithm A }\end{array}$ & IVIF values & IVIF values & $z_{1}>z_{3}>z_{2}>z_{4}$ & Outranking model \\
\hline & $\begin{array}{l}\text { Likelihood-based assignment method } \\
\text { with Algorithm B }\end{array}$ & IVIF values & Incomplete information & $z_{1}>z_{3}>z_{2}>z_{4}$ & Outranking model \\
\hline Chen [42] & IVPF outranking method & IVPF values & IVPF values & $z_{1}>z_{3}>z_{4}>z_{2}$ & Outranking model \\
\hline \multirow{4}{*}{$\begin{array}{l}\text { The current } \\
\text { paper }\end{array}$} & $\begin{array}{l}\text { IVPF TOPSIS method with respect to } \\
\text { displaced ideals }\end{array}$ & IVPF values & $\begin{array}{l}\text { Ordinary (crisp and } \\
\text { nonfuzzy) values }\end{array}$ & $z_{3}>z_{2}>z_{1}>z_{4}$ & Compromise model \\
\hline & $\begin{array}{l}\text { IVPF TOPSIS method with respect to } \\
\text { fixed ideals }\end{array}$ & IVPF values & $\begin{array}{l}\text { Ordinary (crisp and } \\
\text { nonfuzzy) values }\end{array}$ & $z_{3}>z_{1}>z_{2}>z_{4}$ & Compromise model \\
\hline & $\begin{array}{l}\text { Proposed IVPF compromise approach } \\
\text { with Algorithm I }\end{array}$ & IVPF values & $\begin{array}{l}\text { Ordinary (crisp and } \\
\text { nonfuzzy) values }\end{array}$ & $z_{3}>z_{1}>z_{2}>z_{4}$ & Compromise model \\
\hline & $\begin{array}{l}\text { Proposed IVPF compromise approach } \\
\text { with Algorithm II }\end{array}$ & IVPF values & $\begin{array}{l}\text { Ordinary (crisp and } \\
\text { nonfuzzy) values }\end{array}$ & $z_{3}>z_{1}>z_{2}>z_{4}$ & Compromise model \\
\hline
\end{tabular}

criterion $c_{j} \in C_{\mathrm{I}}$, the larger the $S\left(\tilde{\rho}_{i j}\right)$ value is, the better the weighted performance is on $\tilde{\rho}_{i j}$, and the greater the preference is for $\tilde{p}_{i j}$. With respect to a cost criterion $c_{j} \in C_{\mathrm{II}}$, the smaller the $S\left(\tilde{\rho}_{i j}\right)$ value is, the better the weighted performance is on $\tilde{\rho}_{i j}$, and the greater the preference is for $\tilde{p}_{i j}$.

Consider the weight vector $\mathrm{w}^{T}=(0.1404,0.1252,0.1090$ $, 0.0839,0.1361,0.1252,0.1408,0.1394)$ and the IVPF evaluative ratings $\tilde{p}_{1 j}$ and $\tilde{p}_{3 j}$ of $z_{1}$ and $z_{3}$, respectively, with respect to each $c_{j} \in C$ (see Table 1). Employing (49), the extended score functions of $z_{1}$ in relation to each $c_{j}$ were computed as follows: $S\left(\tilde{\rho}_{11}\right)=0.0785, S\left(\tilde{\rho}_{12}\right)=0.2072, S\left(\tilde{\rho}_{13}\right)=0.0087$, $S\left(\tilde{\rho}_{14}\right)=0.0587, S\left(\tilde{\rho}_{15}\right)=0.2433, S\left(\tilde{\rho}_{16}\right)=0.0093, S\left(\tilde{\rho}_{17}\right)=$ 0.0784 , and $S\left(\tilde{\rho}_{18}\right)=0.0011$. Moreover, the extended score functions of $z_{3}$ in terms of each $c_{j}$ were derived as follows: $S$ $\left(\tilde{\rho}_{31}\right)=0.3221, S\left(\tilde{\rho}_{32}\right)=0.0083, S\left(\tilde{\rho}_{33}\right)=0.0396, S\left(\tilde{\rho}_{34}\right)=$ $0.0547, S\left(\tilde{\rho}_{35}\right)=0.2433, S\left(\tilde{\rho}_{36}\right)=0.1009, S\left(\tilde{\rho}_{37}\right)=0.0108$, and $S\left(\tilde{\rho}_{38}\right)=0.0623$. Recall the set of benefit criteria $C_{\mathrm{I}}=$ $\left\{c_{1}, c_{5}\right\}$ and the set of cost criteria $C_{\mathrm{II}}=\left\{c_{2}, c_{3}, c_{4}, c_{6}, c_{7}, c_{8}\right\}$. According to the obtained $S\left(\tilde{\rho}_{1 j}\right)$ and $S\left(\tilde{\rho}_{3 j}\right)$ values, the following comparison results can be acquired. Specifically, $z_{1}$ performs better than $z_{3}$ with respect to $c_{3}, c_{6}$, and $c_{8}$ because $S\left(\tilde{\rho}_{13}\right)<S\left(\tilde{\rho}_{33}\right), S\left(\tilde{\rho}_{16}\right)<S\left(\tilde{\rho}_{36}\right)$, and $S\left(\tilde{\rho}_{18}\right)<S\left(\tilde{\rho}_{38}\right)$, respectively. In contrast, $z_{3}$ performs better than $z_{1}$ with respect to $c_{1}, c_{2}, c_{4}$, and $c_{7}$ because $S\left(\tilde{\rho}_{31}\right)>S\left(\tilde{\rho}_{11}\right), S\left(\tilde{\rho}_{32}\right)<$ $S\left(\tilde{\rho}_{12}\right), S\left(\tilde{\rho}_{34}\right)<S\left(\tilde{\rho}_{14}\right)$, and $S\left(\tilde{\rho}_{37}\right)<S\left(\tilde{\rho}_{17}\right)$, respectively. Additionally, the result $S\left(\tilde{\rho}_{15}\right)=S\left(\tilde{\rho}_{35}\right)$ indicates an indifferent preference between $z_{1}$ and $z_{3}$ with respect to $c_{5}$. Thus, $\tilde{\rho}_{3 j}$ is better than $\tilde{\rho}_{1 j}$ (or, equivalently, $z_{3}$ is preferred to $z_{1}$ on $c_{j}$ ) in terms of four criteria, whereas $\tilde{\rho}_{3 j}$ is worse than $\tilde{\rho}_{1 j}$ (or, equivalently, $z_{3}$ is less preferred to $z_{1}$ on $c_{j}$ ) in terms of three criteria. In particular, $z_{3}$ performs better than $z_{1}$ with respect to the two most important criteria $c_{1}$ and $c_{7}$. Based on the above analysis, the ranking result $z_{3}>z_{1}$ yielded by the compromise-based methods is more appropriate and reasonable than the $z_{1}>z_{3}$ generated by the assignmentbased methods.

Furthermore, the employment of the IVPF TOPSIS method produced different ranking results, i.e., $z_{3}>z_{2}>$ $z_{1}>z_{4}$ and $z_{3}>z_{1}>z_{2}>z_{4}$, with respect to anchored judgments with displaced ideals and fixed ideals, respectively. In contrast, the proposed IVPF compromise approach with Algorithms 1 and 2 acquired the same ranking result $z_{3}>$ $z_{1}>z_{2}>z_{4}$ in relation to displaced ideals and fixed ideals. Thus, concerning the comparative methods of the compromise model, the proposed IVPF compromise approach with Algorithms 1 and 2 is capable of acquiring a stable and credible result in comparison with the IVPF TOPSIS methods.

5.3. Further Studies in Other Application Fields. This subsection attempts to employ the proposed methodology to investigate two real-world cases consisting of a financing decision of working capital policies and a risk evaluation problem of technological innovation to show its practicality and effectiveness in other application fields.

The first application filed focuses on a financial decisionmaking problem. Chen [44] developed an outranking-based MCDA method that utilizes a risk attitudinal assignment model based on an extended concept of risk attitude-based score functions within the PF and IVPF decision environments. The practical usefulness and contributions of the risk attitudinal assignment model have been adequately supported by a financial decision-making application. In this paper, the comparative study attempts to employ the proposed IVPF compromise approach to solve the same selection problem regarding financing policies for working 
capital management within the IVPF environment. This financial decision-making problem, originally introduced by Chen [44], is defined by five financing policies and six criteria for evaluating the alternatives. The five alternatives contain the aggressive dominant policy $\left(z_{1}\right)$, the aggressiveleaning policy $\left(z_{2}\right)$, the balanced aggressive and conservative policy $\left(z_{3}\right)$, the conservative-leaning policy $\left(z_{4}\right)$, and the conservative dominant policy $\left(z_{5}\right)$. The six evaluative criteria include cash reserves $\left(c_{1}\right)$, maturity hedging $\left(c_{2}\right)$, interest rate fluctuation $\left(c_{3}\right)$, financial leverage $\left(c_{4}\right)$, return on assets $\left(c_{5}\right)$, and financing cost $\left(c_{6}\right)$, where the weight vector $\mathrm{w}^{T}=\left(w_{1}, w_{2}, \cdots, w_{6}\right)^{T}=(0.10,0.05,0.20,0.10,0.25$, $0.30)^{T}$. Table 4 presents the data of the $\tilde{p}_{i j}$ values in the IVPF decision matrix $\tilde{p}=\left[\tilde{p}_{i j}\right]_{5 \times 6}$.

Employing the developed IVPF compromise approach to solve the financing problem, the top part of Table 5 indicates the obtained results consisting of the correlations $R\left(\tilde{P}_{i}, \tilde{P}_{*}\right)$ and $R\left(\tilde{P}_{i}, \tilde{P}_{\#}\right)$, the correlation coefficients $K\left(\tilde{P}_{i}, \tilde{P}_{*}\right)$ and $K\left(\tilde{P}_{i}, \tilde{P}_{\#}\right)$, and the correlation-based closeness index with displaced ideals $I_{d}\left(\tilde{P}_{i}\right)$ yielded by the proposed Algorithm 1 , as well as the correlation-based closeness index with fixed ideals $I_{f}\left(\tilde{P}_{i}\right)$ yielded by the proposed Algorithm 2. According to the $I_{d}\left(\tilde{P}_{i}\right)$ values, the ultimate priority ranking $z_{3}>z_{2}>$ $z_{4}>z_{1}>z_{5}$ was acquired based on anchored judgments with displaced ideals. Moreover, the ultimate priority ranking $z_{3}>z_{4}>z_{2}>z_{1}>z_{5}$ was determined by sorting each $I_{f}\left(\tilde{P}_{i}\right)$ value based on anchored judgments with fixed ideals. The best policy is the balanced aggressive and conservative policy $\left(z_{3}\right)$, which is the same as that obtained using the outranking approach based on a risk attitudinal assignment model by Chen [44].

It is noted that Chen [44] conducted a sensitivity analysis to explore the influence of the risk attitudinal parameter. As a whole, the ultimate priority ranking results $z_{3}>z_{4}>z_{2}>$ $z_{5}>z_{1}, z_{3}>z_{4}>z_{2}>z_{1}>z_{5}$, and $z_{1}>z_{4}>z_{3}>z_{2}>z_{5}$ were determined in risk-averse, risk-neutral, and risk-seeking situations, respectively. Figure 4 depicts the comparisons of the ranking results yielded by the proposed methods (with Algorithms 1 and 2) and the risk attitude-based outranking approach (with risk-averse, risk-neutral, and risk-seeking attitudes). It can be observed that the ranking results yielded by the proposed Algorithms 1 and 2 are very similar to those produced by the outranking approach with the risk-averse and risk-neutral attitudes. Moreover, the alternative $z_{3}$ is the best ranked in these results. Based on the comparison outcomes in the financing decision problem, the proposed methodology is capable of generating intuitively reasonable and acceptable results in a more straightforward and effective way, especially the developed Algorithm 2.

The second application filed focuses on a risk evaluation of technological innovation. Chen [63] introduced a novel risk evaluation method of technological innovation using an inferior ratio-based assignment model based on IVPF sets. A real-world case concerning a risk evaluation problem of technological innovation in high-tech enterprises was investigated to examine the feasibility and advantages of the IVPF inferior ratio-based assignment method. In this risk evaluation problem, the set of alternatives contains four candidate projects, consisting of $z_{1}$ (the first potential hightech enterprise), $z_{2}$ (the second potential high-tech enterprise), $z_{3}$ (the third potential high-tech enterprise), and $z_{4}$ (the fourth potential high-tech enterprise). These candidate projects would be evaluated using six risk indicators, including policy risk $\left(c_{1}\right)$, financial risk $\left(c_{2}\right)$, technological risk $\left(c_{3}\right)$, production risk $\left(c_{4}\right)$, market risk $\left(c_{5}\right)$, and managerial risk $\left(c_{6}\right)$. Moreover, the weight vector $\mathrm{w}^{T}=\left(w_{1}, w_{2}, \cdots, w_{6}\right)^{T}=$ $(0.1894,0.1841,0.1361,0.1257,0.1753,0.1894)^{T}$. Table 6 provides the data of the $\tilde{p}_{i j}$ values in the IVPF decision matrix $\tilde{\mathrm{p}}=\left[\tilde{p}_{i j}\right]_{4 \times 6}$. Using the proposed methodology to solve this risk evaluation problem, the obtained results yielded by Algorithms 1 and 2 are revealed in the bottom part of Table 5. Furthermore, Figure 5 contrasts the ranking results yielded by the proposed methods (with Algorithms 1 and 2) and the IVPF inferior ratio-based assignment method.

The ultimate priority ranking $z_{4}>z_{2}>z_{1}>z_{3}$ was determined based on the $I_{d}\left(\tilde{P}_{i}\right)$ values under anchored judgments with displaced ideals. Additionally, the ultimate priority ranking $z_{2}>z_{4}>z_{1}>z_{3}$ was acquired based on the $I_{f}\left(\tilde{P}_{i}\right)$ values under anchored judgments with fixed ideals. It is noted that the ranking result $z_{2}>z_{4}>z_{3}>z_{1}$ was obtained using the IVPF inferior ratio-based assignment method. According to Chen's [63] solution result, the best project is the second potential high-tech enterprise $\left(z_{2}\right)$, which is the same as that obtained using the proposed methodology based on anchored judgments with fixed ideals. In particular, a different result of $z_{4}>z_{2}>z_{1}>z_{3}$ was derived under anchored judgments with displaced ideals. Based on the results in Table 5 and Figure 5, it can be concluded that the proposed methodology can generate acceptable and flexible results through an appropriate employment of the proposed Algorithms 1 and 2.

5.4. Concluding Remarks. In addition to the selection problem of construction methods for bridge superstructures, this paper investigated two real-world cases consisting of a financing decision of working capital policies and a risk evaluation problem of technological innovation to extend the applicability of the proposed methods to other application fields. Based on the comparative studies between the proposed methods and the existing approaches, some valuable observations were obtained and concluded.

First, the feasibility and applicability of the proposed methodology were demonstrated via three practical applications: bridge construction methods for the Suhua Highway Alternative Road Project, financing policies for working capital management, and risk assessments for technological innovation in high-tech enterprises. Additionally, for the bridge construction case, as opposed to the comparative methods of the outranking model in the IVIF context, the proposed methodology produces an evidently reasonable ranking result and performs well in a more complex decision situation, i.e., the IVPF context. As mentioned previously, the space of an IVPF value is larger than the space of an IVIF value because of the relaxed constraint condition, implying that the proposed methodology can sufficiently 
TABLE 4: IVPF evaluative ratings in the financing decision of working capital policies.

\begin{tabular}{|c|c|c|c|c|c|c|}
\hline$c_{j}$ & $z_{i}$ & $\tilde{p}_{i j}$ & $\pi_{i j}$ & $z_{i}$ & $\tilde{p}_{i j}$ & $\pi_{i j}$ \\
\hline$c_{1}$ & $z_{1}$ & $([0.15,0.26],[0.74,0.92])$ & {$[0.29,0.66]$} & $z_{2}$ & $([0.27,0.44],[0.66,0.82])$ & {$[0.37,0.70]$} \\
\hline$c_{2}$ & & $([0.29,0.37],[0.75,0.81])$ & {$[0.45,0.59]$} & & $([0.49,0.61],[0.38,0.58])$ & {$[0.54,0.78]$} \\
\hline$c_{3}$ & & $([0.42,0.56],[0.48,0.59])$ & {$[0.58,0.77]$} & & $([0.53,0.68],[0.42,0.47])$ & {$[0.56,0.74]$} \\
\hline$c_{4}$ & & $([0.22,0.36],[0.64,0.76])$ & {$[0.54,0.74]$} & & $([0.34,0.48],[0.59,0.67])$ & {$[0.57,0.73]$} \\
\hline$c_{5}$ & & $([0.85,0.96],[0.07,0.19])$ & {$[0.21,0.52]$} & & $([0.74,0.86],[0.12,0.21])$ & {$[0.47,0.66]$} \\
\hline$c_{6}$ & & $([0.41,0.56],[0.34,0.45])$ & {$[0.70,0.85]$} & & $([0.67,0.79],[0.23,0.38])$ & {$[0.48,0.71]$} \\
\hline$c_{1}$ & $z_{3}$ & $([0.57,0.69],[0.46,0.57])$ & {$[0.45,0.68]$} & $z_{4}$ & $([0.78,0.86],[0.13,0.28])$ & {$[0.43,0.61]$} \\
\hline$c_{2}$ & & $([0.71,0.82],[0.19,0.30])$ & {$[0.49,0.68]$} & & $([0.78,0.88],[0.11,0.19])$ & {$[0.44,0.62]$} \\
\hline$c_{3}$ & & $([0.84,0.91],[0.15,0.27])$ & {$[0.31,0.52]$} & & $([0.73,0.82],[0.21,0.33])$ & {$[0.47,0.65]$} \\
\hline$c_{4}$ & & $([0.55,0.67],[0.41,0.53])$ & {$[0.52,0.73]$} & & $([0.68,0.79],[0.27,0.34])$ & {$[0.51,0.68]$} \\
\hline$c_{5}$ & & $([0.68,0.74],[0.26,0.35])$ & {$[0.57,0.69]$} & & $([0.28,0.35],[0.72,0.86])$ & {$[0.37,0.63]$} \\
\hline$c_{6}$ & & $([0.88,0.95],[0.09,0.13])$ & {$[0.28,0.47]$} & & $([0.81,0.87],[0.11,0.25])$ & {$[0.42,0.58]$} \\
\hline$c_{1}$ & $z_{5}$ & $([0.92,0.96],[0.11,0.17])$ & {$[0.22,0.38]$} & & & \\
\hline$c_{2}$ & & $([0.89,0.91],[0.05,0.15])$ & {$[0.39,0.45]$} & & & \\
\hline$c_{3}$ & & $([0.56,0.66],[0.38,0.45])$ & {$[0.60,0.74]$} & & & \\
\hline$c_{4}$ & & $([0.78,0.86],[0.23,0.29])$ & {$[0.42,0.58]$} & & & \\
\hline$c_{5}$ & & $([0.07,0.14],[0.82,0.93])$ & {$[0.34,0.57]$} & & & \\
\hline$c_{6}$ & & $([0.43,0.59],[0.52,0.65])$ & {$[0.48,0.74]$} & & & \\
\hline
\end{tabular}

TABLE 5: Selected results in other application fields.

\begin{tabular}{|c|c|c|c|c|c|c|c|c|}
\hline \multirow[b]{2}{*}{$z_{i}$} & \multicolumn{6}{|c|}{ Algorithm 1} & \multicolumn{2}{|c|}{ Algorithm 2} \\
\hline & $R\left(\tilde{P}_{i}, \tilde{P}_{*}\right)$ & $R\left(\tilde{P}_{i}, \tilde{P}_{\#}\right)$ & $K\left(\tilde{P}_{i}, \tilde{P}_{*}\right)$ & $K\left(\tilde{P}_{i}, \tilde{P}_{\#}\right)$ & $I_{d}\left(\tilde{P}_{i}\right)$ & Rank & $I_{f}\left(\tilde{P}_{i}\right)$ & Rank \\
\hline \multicolumn{9}{|c|}{ Results for the financing decision of working capital policies } \\
\hline$z_{1}$ & 0.3718 & 0.3408 & 0.6141 & 0.6741 & 0.4767 & 4 th & 0.5705 & 4 th \\
\hline$z_{2}$ & 0.4458 & 0.3158 & 0.7910 & 0.6710 & 0.5410 & $2 \mathrm{nd}$ & 0.7062 & $3 \mathrm{rd}$ \\
\hline$z_{3}$ & 0.5768 & 0.2666 & 0.9283 & 0.5138 & 0.6437 & $1 \mathrm{st}$ & 0.8770 & $1 \mathrm{st}$ \\
\hline$z_{4}$ & 0.4808 & 0.3528 & 0.7942 & 0.6980 & 0.5322 & $3 \mathrm{rd}$ & 0.7207 & $2 \mathrm{nd}$ \\
\hline$z_{5}$ & 0.3550 & 0.3904 & 0.5883 & 0.7746 & 0.4316 & 5 th & 0.5100 & 5 th \\
\hline \multicolumn{9}{|c|}{ Results for the risk evaluation problem of technological innovation } \\
\hline$z_{1}$ & 0.4858 & 0.3877 & 0.9447 & 0.8738 & 0.5195 & $3 \mathrm{rd}$ & 0.8006 & $3 \mathrm{rd}$ \\
\hline$z_{2}$ & 0.4921 & 0.3825 & 0.9423 & 0.8488 & 0.5261 & 2 nd & 0.8424 & $1 s t$ \\
\hline$z_{3}$ & 0.4715 & 0.3798 & 0.9435 & 0.8807 & 0.5172 & 4 th & 0.7779 & 4 th \\
\hline$z_{4}$ & 0.4968 & 0.3840 & 0.9719 & 0.8705 & 0.5275 & $1 \mathrm{st}$ & 0.8206 & 2nd \\
\hline
\end{tabular}

depict more uncertainty and ambiguity inherent in the subjective judgment and decision-making process by utilizing IVPF information. Furthermore, the usefulness and advantages of the proposed methodology were justified and supported based on the comprehensive comparative analysis. In summary, the proposed IVPF compromise approach leads to both better explanations of and better applicability in addressing MCDA problems because it employs a straightforward and easily implemented decision-making mechanism using correlation-based closeness indices to handle high-order uncertainties associated with practical decision environments.

\section{Conclusions}

This paper focused on the development of a novel compromise model and relevant techniques within the IVPF environment to address highly uncertain MCDA problems, along with an application to bridge construction methods. Bridges are a crucial part of road networks because they can 


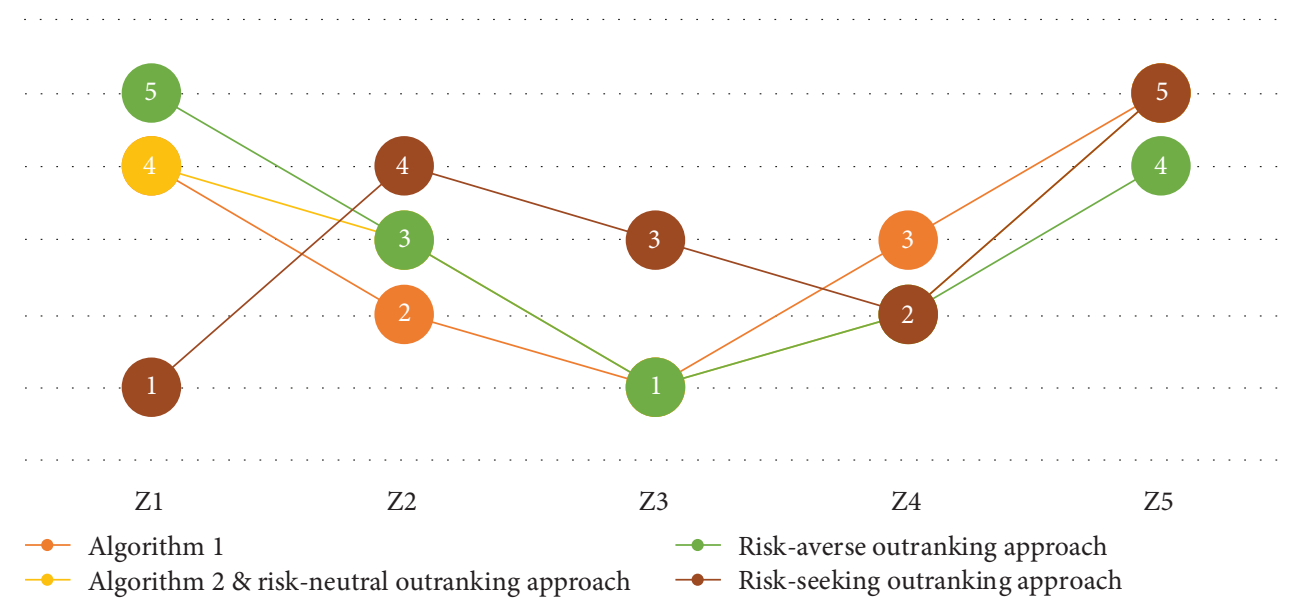

FIgURE 4: Contrast of ultimate priority rankings in the financing decision problem.

TABLE 6: IVPF evaluative ratings in the risk evaluation problem of technological innovation.

\begin{tabular}{|c|c|c|c|c|c|c|}
\hline$c_{j}$ & $z_{i}$ & $\tilde{p}_{i j}$ & $\pi_{i j}$ & $z_{i}$ & $\tilde{p}_{i j}$ & $\pi_{i j}$ \\
\hline$c_{1}$ & $z_{1}$ & $([0.7481,0.8553],[0.2000,0.3000])$ & {$[0.4224,0.6327]$} & $z_{2}$ & $([0.5687,0.7000],[0.2521,0.4401])$ & {$[0.5624,0.7830]$} \\
\hline$c_{2}$ & & $([0.5964,0.8473],[0.1741,0.3680])$ & {$[0.3830,0.7836]$} & & $([0.5252,0.7011],[0.2558,0.4401])$ & {$[0.5610,0.8116]$} \\
\hline$c_{3}$ & & $([0.5755,0.7789],[0.2998,0.4874])$ & {$[0.3947,0.7609]$} & & $([0.6819,0.8731],[0.2549,0.3824])$ & {$[0.3024,0.6856]$} \\
\hline$c_{4}$ & & $([0.7226,0.8439],[0.2921,0.3955])$ & {$[0.3625,0.6265]$} & & $([0.7311,0.8422],[0.1569,0.3000])$ & {$[0.4480,0.6640]$} \\
\hline$c_{5}$ & & $([0.6801,0.8277],[0.2378,0.3770])$ & {$[0.4157,0.6935]$} & & $([0.5964,0.7359],[0.1923,0.4076])$ & {$[0.5407,0.7793]$} \\
\hline$c_{6}$ & & $([0.4957,0.6645],[0.4373,0.5720])$ & {$[0.4809,0.7504]$} & & $([0.8000,0.9000],[0.1516,0.3000])$ & {$[0.3162,0.5805]$} \\
\hline$c_{1}$ & $z_{3}$ & $([0.5284,0.6676],[0.4000,0.5000])$ & {$[0.5516,0.7489]$} & $z_{4}$ & $([0.6552,0.8314],[0.1845,0.3680])$ & {$[0.4164,0.7326]$} \\
\hline$c_{2}$ & & $([0.7656,0.8687],[0.2639,0.3680])$ & {$[0.3316,0.5867]$} & & $([0.7459,0.8492],[0.3031,0.4076])$ & {$[0.3357,0.5931]$} \\
\hline$c_{3}$ & & $([0.6007,0.7541],[0.2521,0.4025])$ & {$[0.5190,0.7587]$} & & $([0.6446,0.8000],[0.4000,0.5000])$ & {$[0.3317,0.6515]$} \\
\hline$c_{4}$ & & $([0.6446,0.8277],[0.2297,0.4345])$ & {$[0.3551,0.7292]$} & & $([0.6248,0.7904],[0.2639,0.3680])$ & {$[0.4897,0.7348]$} \\
\hline$c_{5}$ & & $([0.6454,0.7776],[0.2378,0.3568])$ & {$[0.5177,0.7259]$} & & $([0.5708,0.7515],[0.1414,0.3568])$ & {$[0.5549,0.8088]$} \\
\hline$c_{6}$ & & $([0.6692,0.7703],[0.4000,0.5329])$ & {$[0.3502,0.6262]$} & & $([0.6639,0.7977],[0.3031,0.4076])$ & {$[0.4445,0.6836]$} \\
\hline
\end{tabular}

overcome terrain obstacles and promote regional economic development. Many transportation-related construction projects rely on the use of bridges to join two disconnected regions. However, bridge construction entails challenging technical operations, particularly in regions featuring complex geological structures or frequent natural disasters. Because the criteria for assessing such constructions are numerous and complex, selecting an appropriate bridge construction method involves difficult, multiple-criteria decision-making problems. This paper developed an effective IVPF compromise approach by means of novel correlation-based closeness indices with respect to each IVPF characteristic for assessing all candidate alternatives. This paper charted the landscape of IVPF theory in the compromise decision-making methodology. Moreover, the proposed methods provide a simple and effective means of handling impressions and quantifying the ambiguous nature of human judgments.

For illustrative applications, a real-world case study concerning bridge construction methods was investigated to assist the authority in making an appropriate decision for the Suhua Highway Alternative Road Project and to verify the feasibility and practical effectiveness of the proposed methodology. Furthermore, a comparative analysis was conducted to examine the results yielded by the proposed Algorithms 1 and 2 in comparison with other MCDA approaches (consisting of some nonstandard fuzzy models using the assignment-based outranking methodology and an IVPF extension of the widely used TOPSIS methodology). The obtained results demonstrated the comprehensiveness and dependability of the proposed methods and techniques, particularly the developed Algorithm 2, because of easy implementation and reasonable outcomes. In particular, the usage of the proposed correlation-based closeness indices can effectively facilitate anchored judgments with displaced and fixed ideals, respectively, for each alternative. Additionally, they are useful and practical comparison indices that can reflect certain balances between the connection with the displaced/fixed positive-ideal IVPF solutions and the remotest connection with the displaced/fixed negative-ideal IVPF solutions. With the increasing complexity of MCDA 


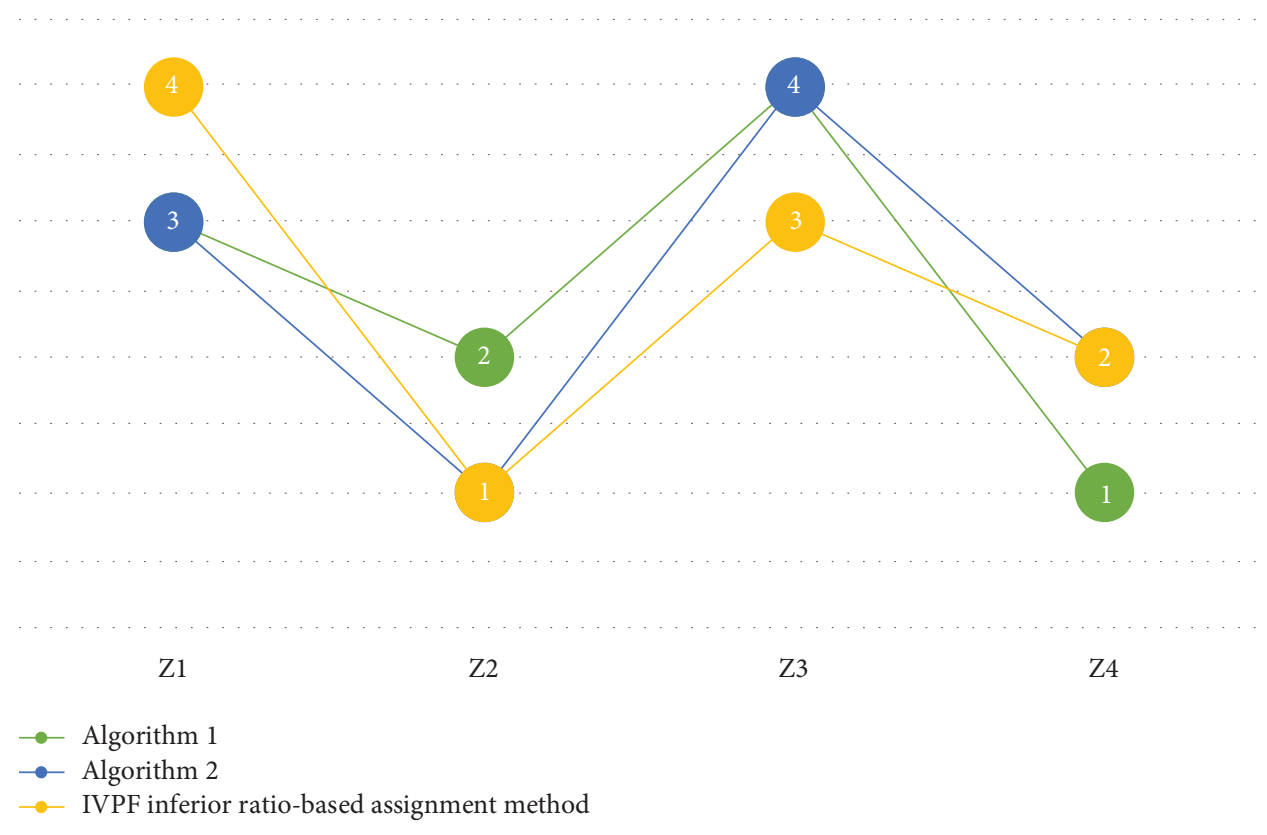

FIGURE 5: Contrast of ultimate priority rankings in the risk evaluation problem.

problems in the real world, the proposed methodology based on the IVPF compromise approach is powerful at handling higher degrees of uncertainty and addressing complicated and changeable MCDA issues using a simple and easy-touse decision-making framework, particularly Algorithm 2, for decision-makers.

Based on the obtained results of the real-world application and comparative studies, this paper has some theoretical and practical implications. First, the developed IVPF compromise approaches can be used to address MCDA problems of bridge construction methods within a highly complex uncertain environment based on IVPF sets. Second, the proposed methodology is capable of enriching the existing compromise-based methods to accommodate more complicated scenarios because it successfully extended the applicability of the compromise model to IVPF contexts. Third, the proposed methodology can be applied to a variety of MCDA fields because it establishes an effective and flexibility mechanism via the employment of IVPF theory to handle complex vagueness and uncertainty in realistic decisionmaking processes.

The current paper successfully proposed a new compromise method that uses the concept of correlation-based closeness indices to manage MCDA problems involving IVPF information. However, a possible limitation exists in the case that the problems characterized by interdependent criteria and dimensions may exhibit feedback-like effects $[64,65]$. Since the current techniques are more appropriately used to conduct MCDA tasks based on the assumption that most criteria are independent, further investigation into extending the applicability with respect to interdependent criteria with feedback-like effects can be conducted to overcome this limitation of the developed methods.

It is anticipated that the developed IVPF compromise approaches not only complement the existing MCDA methods but also create a new direction for the proliferation of IVPF theory into the field of engineering management. Further studies need to replicate this research in other industry domains and in other decision environments. On the theoretical front, a future research direction can be to investigate the potentials of extending other compromise-based methodologies to the IVPF decision environment.

\section{Data Availability}

The datasets generated during and/or analyzed during the current study are available from the corresponding author on reasonable request.

\section{Conflicts of Interest}

The author declares that there is no conflict of interest regarding the publication of this paper.

\section{Acknowledgments}

The author acknowledges the assistance of the respected editor and the anonymous referees for their insightful and constructive comments, which helped to improve the overall quality of the paper. The author is grateful for the grant funding support from the Taiwan Ministry of Science and Technology (MOST 105-2410-H-182-007-MY3) and Chang Gung Memorial Hospital (BMRP 574 and CMRPD2F0202) during the study completion.

\section{References}

[1] A. Amini, N. Nikraz, and A. Fathizadeh, "Identifying and evaluating the effective parameters in prioritization of urban roadway bridges for maintenance operations," Australian Journal of Civil Engineering, vol. 14, no. 1, pp. 23-34, 2016. 
[2] V. Penadés-Plà, T. García-Segura, J. Martí, and V. Yepes, “A review of multi-criteria decision-making methods applied to the sustainable bridge design," Sustainability, vol. 8, no. 12, p. 1295, 2016.

[3] T. García-Segura and V. Yepes, "Multiobjective optimization of post-tensioned concrete box-girder road bridges considering cost, $\mathrm{CO}_{2}$ emissions, and safety," Engineering Structures, vol. 125, pp. 325-336, 2016.

[4] T. García-Segura, V. Yepes, J. Alcalá, and E. Pérez-López, "Hybrid harmony search for sustainable design of posttensioned concrete box-girder pedestrian bridges," Engineering Structures, vol. 92, pp. 112-122, 2015.

[5] M. S. M. Lund, J. T. Kevern, V. R. Schaefer, and K. K. Hansen, "Mix design for improved strength and freeze-thaw durability of pervious concrete fill in pearl-chain bridges," Materials and Structures, vol. 50, no. 1, 2017.

[6] T.-T. Chen and C.-H. Wang, "Fall risk assessment of bridge construction using Bayesian network transferring from fault tree analysis," Journal of Civil Engineering and Management, vol. 23, no. 2, pp. 273-282, 2017.

[7] M. Skibniewski, H.-P. Tserng, S.-H. Ju et al., "Web-based real time bridge scour monitoring system for disaster management," The Baltic Journal of Road and Bridge Engineering, vol. 9, no. 1, pp. 17-25, 2014.

[8] S. A. Grammatikos, R. J. Ball, M. Evernden, and R. G. Jones, "Impedance spectroscopy as a tool for moisture uptake monitoring in construction composites during service," Composites Part A: Applied Science and Manufacturing, vol. 105, pp. 108117, 2018.

[9] P. Jakiel and D. Fabianowski, "FAHP model used for assessment of highway RC bridge structural and technological arrangements," Expert Systems with Applications, vol. 42, no. 8, pp. 4054-4061, 2015.

[10] J. V. Martí, T. García-Segura, and V. Yepes, "Structural design of precast-prestressed concrete U-beam road bridges based on embodied energy," Journal of Cleaner Production, vol. 120, pp. 231-240, 2016.

[11] V. Yepes, J. V. Martí, and T. García-Segura, "Cost and $\mathrm{CO}_{2}$ emission optimization of precast-prestressed concrete U-beam road bridges by a hybrid glowworm swarm algorithm," Automation in Construction, vol. 49, no. Part A, pp. 123-134, 2015.

[12] D. Jato-Espino, E. Castillo-Lopez, J. Rodriguez-Hernandez, and J. C. Canteras-Jordana, "A review of application of multi-criteria decision making methods in construction," Automation in Construction, vol. 45, pp. 151-162, 2014.

[13] J. Krebs, F. Jacobs, R. Conway et al., "Methods for predicting potential impacts of pile-driving noise on endangered sturgeon during bridge construction," in The Effects of Noise on Aquatic Life II, vol. 875 of Advances in Experimental Medicine and Biology, pp. 565-572, Springer, New York, NY, USA, 2016.

[14] D. Peduto, F. Elia, and R. Montuori, "Probabilistic analysis of settlement-induced damage to bridges in the city of Amsterdam (the Netherlands)," Transportation Geotechnics, vol. 14, pp. 169-182, 2018.

[15] M. Yang, S. Kainuma, and Y.-S. Jeong, "Structural behavior of orthotropic steel decks with artificial cracks in longitudinal ribs," Journal of Constructional Steel Research, vol. 141, pp. 132-144, 2018.

[16] I. Björnsson, "Holistic approach for treatment of accidental hazards during conceptual design of bridges - a case study in Sweden," Safety Science, vol. 91, pp. 168-180, 2017.
[17] G. Pál and K. Hiros, "Pedestrian bridge over Türr-channel Baja, Hungary: a unique arch bridge design," Procedia Engineering, vol. 156, pp. 312-319, 2016.

[18] T.-Y. Chen, "The extended linear assignment method for multiple criteria decision analysis based on interval-valued intuitionistic fuzzy sets," Applied Mathematical Modelling, vol. 38, no. 7-8, pp. 2101-2117, 2014.

[19] A. T. Machwe and I. C. Parmee, "Multi-objective analysis of a component-based representation within an interactive evolutionary design system," Engineering Optimization, vol. 39, no. 5, pp. 591-613, 2007.

[20] L. Xu, Z. Li, S. Li, and F. Tang, "A polychromatic sets approach to the conceptual design of machine tools," International Journal of Production Research, vol. 43, no. 12, pp. 2397-2421, 2005.

[21] H. Malekly, S. Meysam Mousavi, and H. Hashemi, “A fuzzy integrated methodology for evaluating conceptual bridge design," Expert Systems with Applications, vol. 37, no. 7, pp. 4910-4920, 2010.

[22] S. Rana, N. Islam, R. Ahsan, and S. N. Ghani, "Application of evolutionary operation to the minimum cost design of continuous prestressed concrete bridge structure," Engineering Structures, vol. 46, pp. 38-48, 2013.

[23] F. Y. Xu, M. J. Zhang, L. Wang, and J. R. Zhang, "Recent highway bridge collapses in China: review and discussion," Journal of Performance of Constructed Facilities, vol. 30, no. 5, p. $04016030,2016$.

[24] R. Nadafianshahamabadi, M. Tayarani, and G. M. Rowangould, "Differences in expertise and values: comparing community and expert assessments of a transportation project," Sustainable Cities and Society, vol. 28, pp. 67-75, 2017.

[25] W. Bulleit, J. Schmidt, I. Alvi, E. Nelson, and T. RodriguezNikl, "Philosophy of engineering: what it is and why it matters," Journal of Professional Issues in Engineering Education and Practice, vol. 141, no. 3, 2015.

[26] B. Enright, E. J. OBrien, and C. Leahy, "Identifying and modelling permit trucks for bridge loading," Proceedings of the Institution of Civil Engineers - Bridge Engineering, vol. 169, no. 4, pp. 235-244, 2016.

[27] P. Jiříček and M. Foglar, "Numerical analysis of a bridge pier subjected to truck impact," Structural Concrete, vol. 17, no. 6, pp. 936-946, 2016.

[28] V. Mara, M. Al-Emrani, and R. Haghani, "A novel connection for fibre reinforced polymer bridge decks: conceptual design and experimental investigation," Composite Structures, vol. 117, pp. 83-97, 2014.

[29] R. R. Yager, "Pythagorean fuzzy subsets," in 2013 Joint IFSA World Congress and NAFIPS Annual Meeting (IFSA/NAFIPS), pp. 57-61, Edmonton, Canada, June 2013.

[30] R. R. Yager, "Pythagorean membership grades in multicriteria decision making," IEEE Transactions on Fuzzy Systems, vol. 22, no. 4, pp. 958-965, 2014.

[31] R. R. Yager, "Properties and applications of Pythagorean fuzzy sets," in Imprecision and Uncertainty in Information Representation and Processing, P. Angelov and S. Sotirov, Eds., pp. 119136, Springer, Cham, Switzerland, 2016.

[32] R. R. Yager and A. M. Abbasov, "Pythagorean membership grades, complex numbers, and decision making," International Journal of Intelligent Systems, vol. 28, no. 5, pp. 436452, 2013.

[33] A. Biswas and B. Sarkar, "Pythagorean fuzzy multicriteria group decision making through similarity measure based on 
point operators," International Journal of Intelligent Systems, vol. 33, no. 8, pp. 1731-1744, 2018.

[34] T.-Y. Chen, "Remoteness index-based Pythagorean fuzzy VIKOR methods with a generalized distance measure for multiple criteria decision analysis," Information Fusion, vol. 41, pp. 129-150, 2018.

[35] S.-P. Wan, S.-Q. Li, and J.-Y. Dong, "A three-phase method for Pythagorean fuzzy multi-attribute group decision making and application to haze management," Computers \& Industrial Engineering, vol. 123, pp. 348-363, 2018.

[36] X. Zhang and Z. Xu, "Extension of TOPSIS to multiple criteria decision making with Pythagorean fuzzy sets," International Journal of Intelligent Systems, vol. 29, no. 12, pp. 1061-1078, 2014.

[37] H. Garg, "A novel correlation coefficients between Pythagorean fuzzy sets and its applications to decision-making processes," International Journal of Intelligent Systems, vol. 31, no. 12, pp. 1234-1252, 2016.

[38] D. Liang and Z. Xu, "The new extension of TOPSIS method for multiple criteria decision making with hesitant Pythagorean fuzzy sets," Applied Soft Computing, vol. 60, pp. 167-179, 2017.

[39] W. Xue, Z. Xu, X. Zhang, and X. Tian, "Pythagorean fuzzy LINMAP method based on the entropy theory for railway project investment decision making," International Journal of Intelligent Systems, vol. 33, no. 1, pp. 93-125, 2018.

[40] X. Zhang, "Multicriteria Pythagorean fuzzy decision analysis: a hierarchical QUALIFLEX approach with the closeness indexbased ranking methods," Information Sciences, vol. 330, pp. 104-124, 2016.

[41] R. Zhang, J. Wang, X. Zhu, M. Xia, and M. Yu, "Some generalized Pythagorean fuzzy Bonferroni mean aggregation operators with their application to multiattribute group decision-making," Complexity, vol. 2017, Article ID 5937376, 16 pages, 2017.

[42] T.-Y. Chen, “An interval-valued Pythagorean fuzzy outranking method with a closeness-based assignment model for multiple criteria decision making," International Journal of Intelligent Systems, vol. 33, no. 1, pp. 126-168, 2018.

[43] X. Peng and Y. Yang, "Fundamental properties of intervalvalued Pythagorean fuzzy aggregation operators," International Journal of Intelligent Systems, vol. 31, no. 5, pp. 444487, 2016.

[44] T.-Y. Chen, “An outranking approach using a risk attitudinal assignment model involving Pythagorean fuzzy information and its application to financial decision making," Applied Soft Computing, vol. 71, pp. 460-487, 2018.

[45] H. Garg, "A linear programming method based on an improved score function for interval-valued Pythagorean fuzzy numbers and its application to decision-making," International Journal of Uncertainty, Fuzziness and KnowledgeBased Systems, vol. 26, no. 1, pp. 67-80, 2018.

[46] H. Garg, "New exponential operational laws and their aggregation operators for interval-valued Pythagorean fuzzy multicriteria decision-making," International Journal of Intelligent Systems, vol. 33, no. 3, pp. 653-683, 2018.

[47] D. Liang, A. P. Darko, and Z. Xu, "Interval-valued Pythagorean fuzzy extended Bonferroni mean for dealing with heterogenous relationship among attributes," International Journal of Intelligent Systems, vol. 33, no. 7, pp. 1381-1411, 2018.

[48] Y. Liu, Y. Qin, and Y. Han, "Multiple criteria decision making with probabilities in interval-valued Pythagorean fuzzy setting," International Journal of Fuzzy Systems, vol. 20, no. 2, pp. 558-571, 2018.

[49] M. S. A. Khan and S. Abdullah, "Interval-valued Pythagorean fuzzy GRA method for multiple-attribute decision making with incomplete weight information," International Journal of Intelligent Systems, vol. 33, no. 8, pp. 1689-1716, 2018.

[50] H. Garg, "Some methods for strategic decision-making problems with immediate probabilities in Pythagorean fuzzy environment," International Journal of Intelligent Systems, vol. 33, no. 4, pp. 687-712, 2018.

[51] T.-Y. Chen, “An inclusion comparison approach for multiple criteria decision analysis based on interval-valued intuitionistic fuzzy sets," Technological and Economic Development of Economy, vol. 22, no. 3, pp. 357-392, 2016.

[52] J.-C. Wang and T.-Y. Chen, "Likelihood-based assignment methods for multiple criteria decision analysis based on interval-valued intuitionistic fuzzy sets," Fuzzy Optimization and Decision Making, vol. 14, no. 4, pp. 425-457, 2015.

[53] J.-C. Wang and T.-Y. Chen, "A novel multiple criteria decision-making method using a likelihood-based compromise approach under complex uncertainty," International Journal of Research in Engineering and Technology, vol. 6, no. 6, pp. 46-57, 2017.

[54] C.-Y. Tsao and T.-Y. Chen, "A projection-based compromising method for multiple criteria decision analysis with interval-valued intuitionistic fuzzy information," Applied Soft Computing, vol. 45, pp. 207-223, 2016.

[55] R. Liang, Z. Dong, Z. Sheng, X. Wang, and C. Wu, "Case study of selecting decision-making schemes in large-scale infrastructure projects," Journal of Infrastructure Systems, vol. 23, no. 4, 2017.

[56] X. X. Huang and X. M. Wang, "Digital model of road and bridge construction enterprise purchasing and its applied research," Applied Mechanics and Materials, vol. 405-408, pp. 3499-3504, 2013.

[57] Z.-Y. Wang, G.-D. Li, and Y.-H. Wang, "Optimization decision model for bridge design based on AHP-TOPSIS," Journal of Jilin University (Engineering and Technology Edition), vol. 47, no. 2, pp. 478-482, 2017.

[58] N. F. Pan, "Fuzzy AHP approach for selecting the suitable bridge construction method," Automation in Construction, vol. 17, no. 8, pp. 958-965, 2008.

[59] T.-Y. Chen, "Nonlinear assignment-based methods for interval-valued intuitionistic fuzzy multi-criteria decision analysis with incomplete preference information," International Journal of Information Technology \& Decision Making, vol. 11, no. 4, pp. 821-855, 2012.

[60] T.-Y. Chen, "Interval-valued intuitionistic fuzzy QUALIFLEX method with a likelihood-based comparison approach for multiple criteria decision analysis," Information Sciences, vol. 261, pp. 149-169, 2014.

[61] K. Atanassov and G. Gargov, "Interval valued intuitionistic fuzzy sets," Fuzzy Sets and Systems, vol. 31, no. 3, pp. 343349, 1989.

[62] D. Yu, Y. Wu, and T. Lu, "Interval-valued intuitionistic fuzzy prioritized operators and their application in group decision making," Knowledge-Based Systems, vol. 30, pp. 57-66, 2012.

[63] T.-Y. Chen, "A novel risk evaluation method of technological innovation using an inferior ratio-based assignment model in the face of complex uncertainty," Expert Systems with Applications, vol. 95, pp. 333-350, 2018. 
[64] T.-Y. Chen, "An IVIF-ELECTRE outranking method for multiple criteria decision-making with interval-valued intuitionistic fuzzy sets," Technological and Economic Development of Economy, vol. 22, no. 3, pp. 416-452, 2016.

[65] E. K. Zavadskas and Z. Turskis, "Multiple criteria decision making (MCDM) methods in economics: an overview," Technological and Economic Development of Economy, vol. 17, no. 2, pp. 397-427, 2011. 


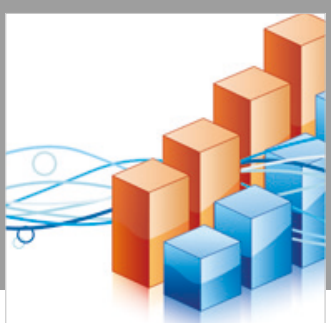

Advances in

Operations Research

\section{-n-m}
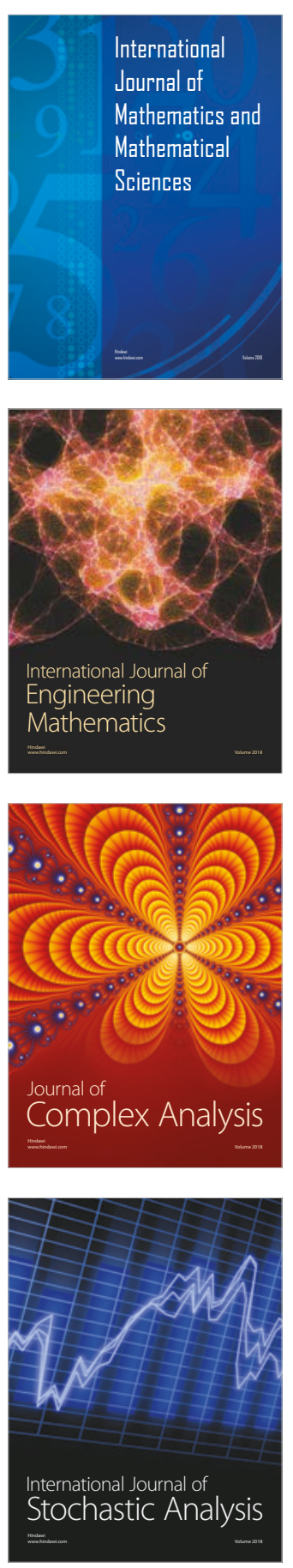
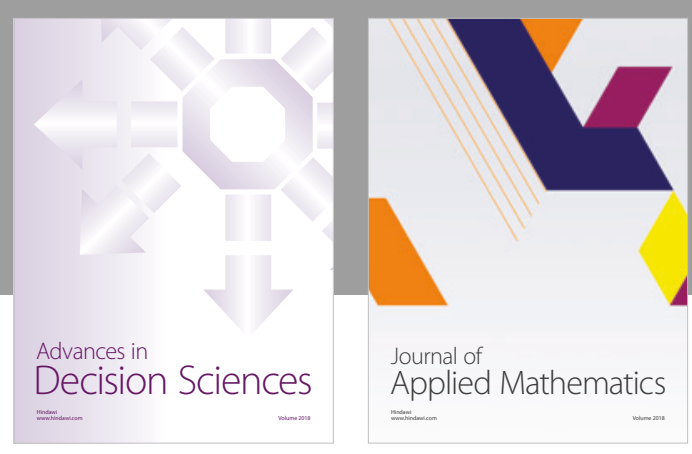

Journal of

Applied Mathematics
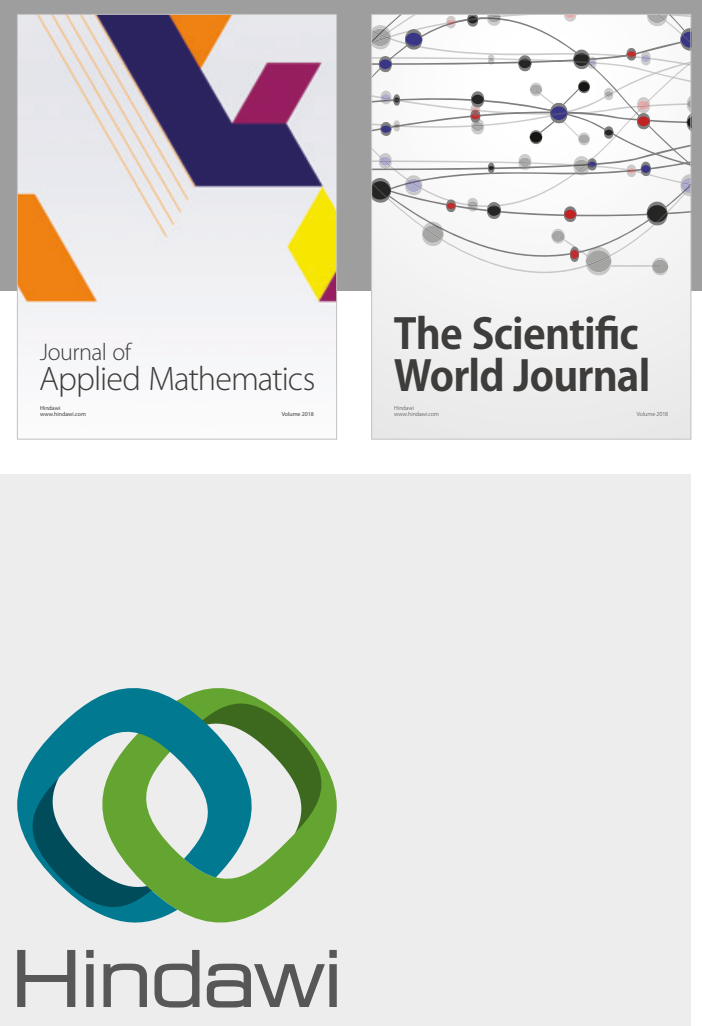

Submit your manuscripts at

www.hindawi.com

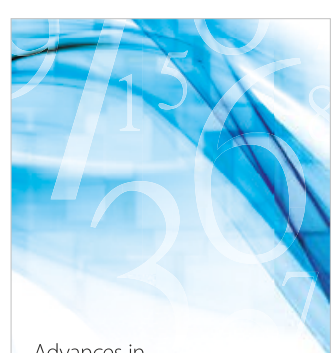

Advances in
Numerical Analysis
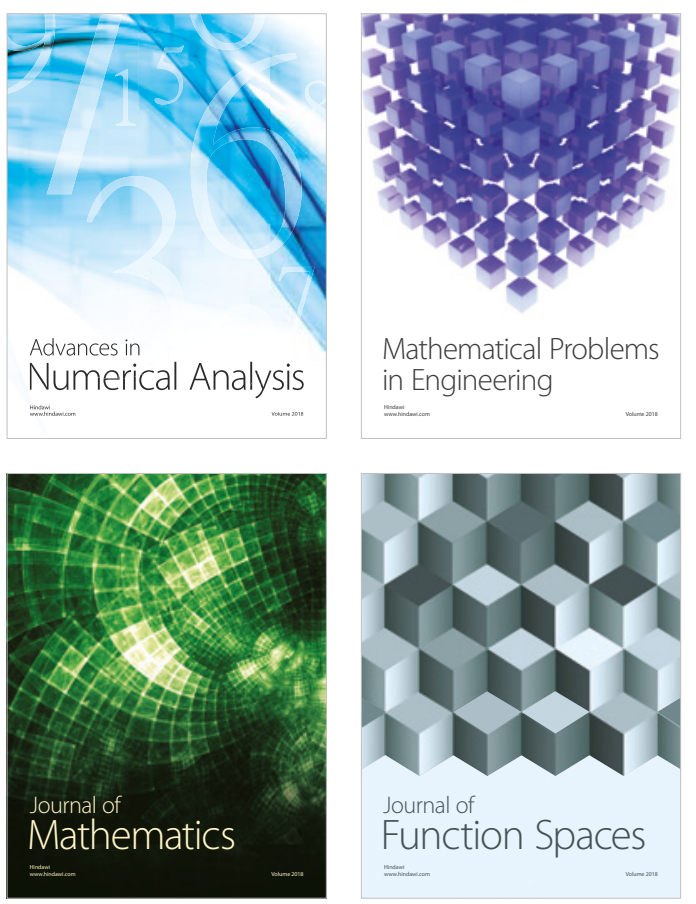

Mathematical Problems in Engineering

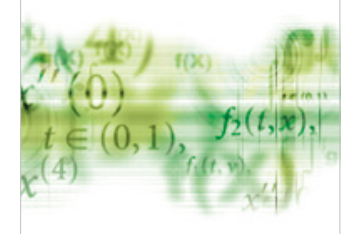

International Journal of

Differential Equations

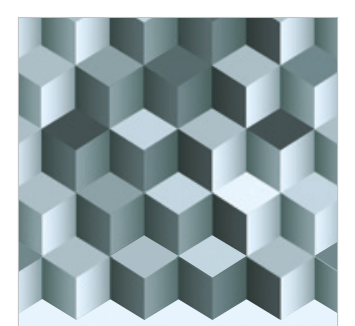

Journal of

Function Spaces
The Scientific

World Journal

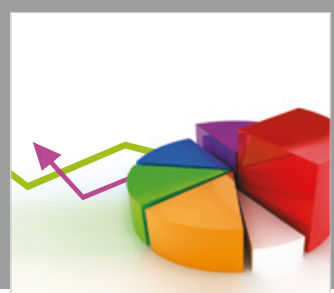

Journal of

Probability and Statistics
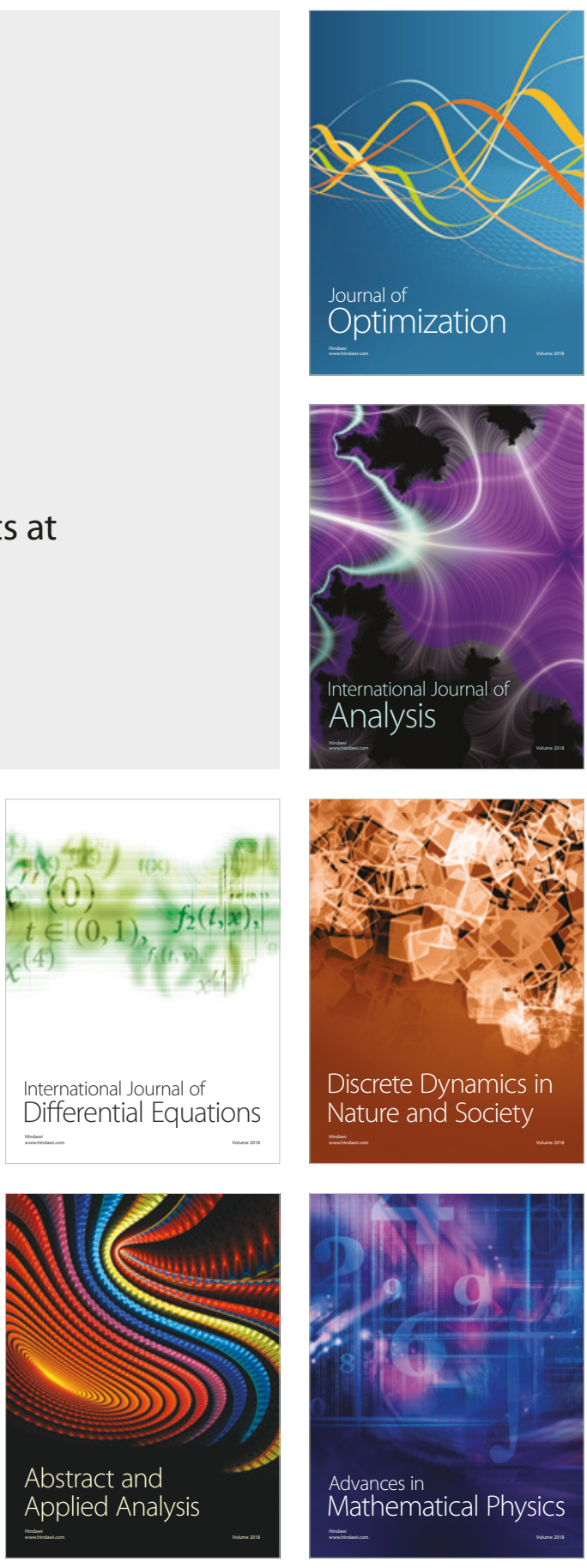